\title{
A New Approach to High-Order Electroencephalogram Phase Analysis Details the Mathematical Mechanisms of Central Nervous System Impulse Encoding
}

\author{
Ricardo J. Simeoni \\ Research and Development, Neurödinger, Sunshine Coast, QLD 4575 Australia. \\ Corresponding author: Ricardo J. Simeoni (rsimeoni@neurodinger.com, www.neurodinger.com). \\ This work is fully funded by Neurödinger which holds an associated patent pending with priority date Sept. 9, 2020.
}

\begin{abstract}
This paper presents a new electroencephalogram (EEG) analysis technique which is applied to example EEGs pertaining to nine human subjects and a broad spectrum of clinical scenarios. While focusing on technique physical efficacy, the paper also paves the way for future clinically-focused studies with revelations of several quantified and detailed findings in relation to high-order central nervous system communicative impulse encoding akin to a sophisticated form of phase-shift keying. The fact that fine encoding details are extracted with confidence from a seemingly modest EEG set supports the paper's position that vast amounts of accessible information currently goes unrecognised by conventional EEG analysis. The technique commences with high resolution Fourier analysis being twice applied to an EEG, providing newly-identified harmonics. Except for deep sleep where harmonic phase, $\phi$, behaviour becomes highly linear, $\phi$ transitional values, $\Delta \phi$, measured between harmonics of progressively increasing order are found to cluster rather than follow a normal distribution (e.g., $\chi^{2}=303, d f=12, p<0.001$ ). Clustering is categorised into ten Families for which many separations between $\Delta \phi$ values are writable in terms of $k=j / 4$ or $j / 3(j=1,2,3 \ldots)$, with a preference for $k=j / 2\left(\chi^{2}=77, d f=1, p<0.001\right)$, amounts of a Family-specific quantum increment value, $\alpha_{\Delta \phi}$. A parabolic relationship $(r>0.9999, p<0.001)$ exists between $\alpha_{\Delta \phi}$ (and the parabola minimum associates with an additional inter-Family or universal quantum increment value, $\alpha_{\text {min }}$ ). Ratios of $\alpha_{\Delta \phi}$ typically align within $\pm 0.5 \%$ of simple common fractions (95\% CI).
\end{abstract}

INDEX TERMS Communication, electroencephalogram, encoding, high-order phase analysis, phase-shift keying

\section{INTRODUCTION}

\subsection{GENERAL INTRODUCTION AND AIMS OF STUDY}

The complexity of the human brain with its approximately 100 billion neurons undoubtedly transcends to some highorder form of collective electrical signal (impulse) encoding within everyday central nervous system (CNS) function associated with motor control and the cognitive processes including those of memory storage, recall and utilisation. The sophisticated detail of this encoding (i.e., the mathematical nature and co-ordination of embedded modulation and sequencing keys) is far from fully understood despite decades of scientific study into the human electroencephalogram (EEG). In fact, given the large volume of information gathered in a single EEG sitting, through multi-channel high sampling rate data acquisition of several minutes to hours duration, modern EEG analysis surprisingly still mostly adopts conventional, in some cases rudimentary, brain activity indicators that are based upon long-standing approaches, or extensions to these core approaches, rather than upon genuinely new analysis alternatives, and this is particularly true at the clinical interface (these existing indicators are introduced within Subsection 1.2).

One may readily undertake any number of contrasting (in terms of information volume provision) colloquial comparisons to appreciate that, while conventional EEG analysis is often clinically diagnostic, vast volumes of untapped CNS impulse encoding information must be embedded within the complexities of the EEG, and so 
remain lost to neurodiagnosis. Two such colloquial comparison examples include $1 \mu \mathrm{g}$ of DNA being theoretically capable of storing up to approximately 500 Tbytes of information [1], and the efficient communication rates ( $\geq$ Mbit per second) of Bluetooth-type technologies [2]. The encoding complexities of the CNS must logically be somewhat comparable (if not superior) to these systems, yet current EEG information revelation pales by comparison. Nonetheless, the cerebral cortex functional activation information (of high temporal resolution) provided by conventional EEG analysis, sometimes in conjunction with magnetoencephalogram (MEG) analysis which originates from the same neurophysiological processes, is clinically invaluable, as is the electrophysiological information provided by sophisticated and established EEG simulation models [3-5] that, through techniques such as inverse solution dipole localisation, provide important EEG insights into organisational complexities of spatiotemporal dynamics across cortical depths, synchronicity, coherence, and surface Laplacians. Note that due to the EEG-MEG overlap, several aspects of the present study will likely also apply to MEG analysis (with recognition of its comparative advantages and disadvantages including challenges associated with accessibility and low-level magnetic field detection), however study focus will remain on EEG analysis.

Functional magnetic resonance imaging (fMRI) and positron emission tomography (PET) increasingly offer competing alternatives or complementary options to EEG analysis, as they collectively detect parameters such as: (i) cerebral blood flow and fluctuations in capillary deoxyhaemoglobin populations which are triggered by the activity of neural populations; (ii) the rate of glucose metabolism which is proportional to the rate of synaptic firing; and (iii) enhanced dopamine secretion which is linked to the activation of genes responsible for brain function [6-11]. For example, through the detection of otherwise finely hidden levels of brain activity, fMRI and (especially) PET offer improved differential diagnosis and long-term recovery likelihood prognostication within consciousness disorders such as unresponsive wakefulness syndrome [8]. Another synergism example challenges a previously held notion of EEG-fMRI interchangeability when analysing neural modulation mechanisms that support visual attention [10]; the demonstration of categorically different stimulus-evoked EEG and fMRI signal patterns in that study leads to a conclusion of necessary haemodynamic and electrophysical system complementarity for the understanding of neural processes supporting cognition. However, despite these described modality synergies, the inherent physical natures of fMRI and PET with their classifications as secondary brain function measures (due to their collective reliance on blood flow and physiological processes involving metabolic activity changes), makes more direct EEG analysis the most likely of the modalities to be receptive to new methods of high-order mathematical analysis with CNS impulse encoding revelation.

The current limited understanding of CNS impulse encoding does not of course negate: (i) the life-changing capacity of advancements in implantable neuroprosthetics such as the cochlear ear [12] and bionic eye (retinal implant) [13]; (ii) the similar capacity of new generation implantable brain-computer interface (BCI) devices including both predictive/advisory-types (e.g., preparing an implanted patient for an anticipated epileptic seizure) and sensory motor function-types (e.g., involving prosthetic limb control) [14-16]; and (iii) the therapeutic effectiveness of deep brain stimulation [17-19], transcranial magnetic stimulation (TMS) [20], and other such neural stimulation therapies.

Rather, the afore introductory comments reasonably recognise that even with a predicted BCI revolution on the neural engineering horizon [15], several years of further advancements are still required to achieve: (i) a full understanding of the deliberate physiology of action potential processes and coordination that lead to EEG characteristics; (ii) neuroprosthetics and BCI at levels allowing full and fine autonomous control (e.g., signal interfacing to substantially mitigate conditions such as spinal cord injury); and (iii) more informed and effective methods of formulating, administering and understanding the benefits of targeted neural stimulation therapies (e.g., for the alleviation treatment of conditions such as depression, Alzheimer's disease, Parkinson's disease, Tourette syndrome, tinnitus, and so on).

The present study primarily aims to contribute to such advancement by the introduction of a new, high-order EEG phase analysis technique via its application to a broad clinical spectrum of EEGs (associated with healthy adults in various awake and sleep states, an adult diagnosed with probable Alzheimer's disease, and various electrode positions), with focus remaining on the physical efficacy and applicability of the technique in the variety of clinical circumstances. That is, the design and implementation of clinical research into some specific group or pathology is not part of the study's aims. However, in reaching its primary aim the study is inevitably expected to generate a basis for such clinical research into the future, and the identification and interpretation of new, high-order characteristics of EEG phase-based encoding is anticipated. In line with [2] it is also postulated that any such phasebased encoding will possess significant quantisation facets.

\subsection{CONVENTIONAL EEG ANALYSIS BRIEF OVERVIEW} Time-domain EEG waveforms routinely provide clinical information such as burst and other pattern diagnostics in response to some external auditory, sensory or visual neural stimulus (e.g., temporary reactive changes in parameters such as amplitude and signal-to-noise ratio for a steady- 
state visually-evoked potential, or SSVEP). Frequencydomain spectra provide a range of complementary diagnostic indices which often refer to the traditional delta, theta, alpha and beta bands, approximately corresponding to spectral frequency ranges of 0.5 to 4,4 to 8,8 to 13 and $>13 \mathrm{~Hz}$ respectively (an additional gamma band that caps and surpasses the beta band from approximately $30 \mathrm{~Hz}$ onwards may also be included for relatively high frequency spectra). The total and proportional activities (or powers) of the traditional bands, as well as several permutations of ratios of band activities are representative of conventional EEG indices that are monitored within a diagnostic setting [11,21-23], with related but more advanced indices formed by combining several frequency band components (e.g., of the SSVEP).

The index of coherence, that measures the degree of phase similarity and thus functional connectivity for different brain locations, as well as long- and short-range correlates between frequency band components for different brain locations, also make valuable contributions to the understanding of brain function (e.g., by demonstrating that smaller amplitude alpha activities in prefrontal and frontal locations are not solely due to the conduction of electrical currents associated with typically larger amplitude alpha activities in occipital-parietal locations, as was once supported) [11,24,25]. Relatively simple indices that are not specific to any particular band include median frequency and spectral edge frequency (SEF), where SEF is defined as the frequency below which a specified percentage (typically 95\%) of a spectrum's power exists [23,26].

It has long been recognised that despite the important clinical information and quantified correlations with physiological or pathological states that conventional EEG indices like those above can provide, their correlations are nevertheless sometimes equivocal or confounded by influencers (e.g., anaesthetic drugs), especially for more simple indices like SEF [23,26-28], such are the inherent complexities of EEG dynamics [4]. Furthermore, even the most experienced EEG reader (e.g., anaesthesiologist, neurologist, sleep technician, etc.) will acknowledge that the neurodiagnostic capability of the EEG overall remains restrictive and certainly lacks specific detailed impulse encoding information as previously introduced. Hence, the development of EEG analysis techniques of ever increasing sophistication is ongoing.

A brief overview of one such sophisticated yet well established phase-based analysis technique, namely bispectral (bicoherence) analysis, follows (since the present study aims to identify and apply a new form of high-order, phase-based EEG analysis, potentially with the inclusion of impulse encoding revelations). However, before that overview it is pertinent to acknowledge that at their core, these established and emerging techniques still incorporate the same wide umbrella of conventional spectral principles, and it may therefore be argued that EEG analysis advancements to some degree are plateauing.

Bispectral analysis, which includes the subset of bicoherence analysis, detects for the existence of phase couplings between the $n$ sinusoidal harmonics, $X_{n}\left(f_{n}\right)=c_{n} \sin \left(2 \pi f_{n} t+\phi_{n}\right)$, of an EEG biosignal, where for a given harmonic $c_{n}$ is amplitude, $f_{n}$ is frequency and $\phi_{n}$ is phase, with $t$ being time. For common second-order analysis, bicoherence, written $\operatorname{bic}\left(f_{p}, f_{q}\right)$, equals $100 \%$ if harmonics $X\left(f_{p}\right), X\left(f_{q}\right)$ and $X\left(f_{p}+f_{q}\right)$ maintain a constant phase relationship in time (see figure 1a). Conversely, if the phases of the three harmonics vary independently in time, as represented by the different phase relationships for the time intervals either side of the vertical line in figure $1 \mathrm{~b}$, then $\operatorname{bic}\left(f_{p}, f_{q}\right)$ equals $0 \%$. Accordingly, $\operatorname{bic}\left(f_{p}, f_{q}\right)$ values between 0 and $100 \%$ represent other proportionate degrees of second-order phase coupling. A graph of all bic $\left(f_{p}, f_{q}\right)$ values gives the bicoherence spectrum which is a normalised (via $c_{n}$ effect negation) version of the bispectrum of corresponding $B\left(f_{p}, f_{q}\right)$ values. To calculate $\operatorname{bic}\left(f_{p}, f_{q}\right)$ (and $B\left(f_{p}, f_{q}\right)$ which is implied by $\operatorname{bic}\left(f_{p}, f_{q}\right)$ from this point) over an extended time period, the EEG biosignal is divided into several (e.g., 32) epochs, which may or may not overlap (two such contiguous epochs are depicted by figure 1b), and the harmonic phase information of all epochs is then statistically combined (see [29-32] for relevant equations and further background).

Relatively high $\operatorname{bic}\left(f_{p}, f_{q}\right)$ values indicate that brain function involves significant nonlinear processes (the brain is considered a nonlinear biological system that displays complex dynamics and deterministic chaotic behaviour) and, by observing changes in second-order phase couplings in response to various stimuli, information on, or a deeper understanding of, these nonlinear processes may be achieved [30-35].
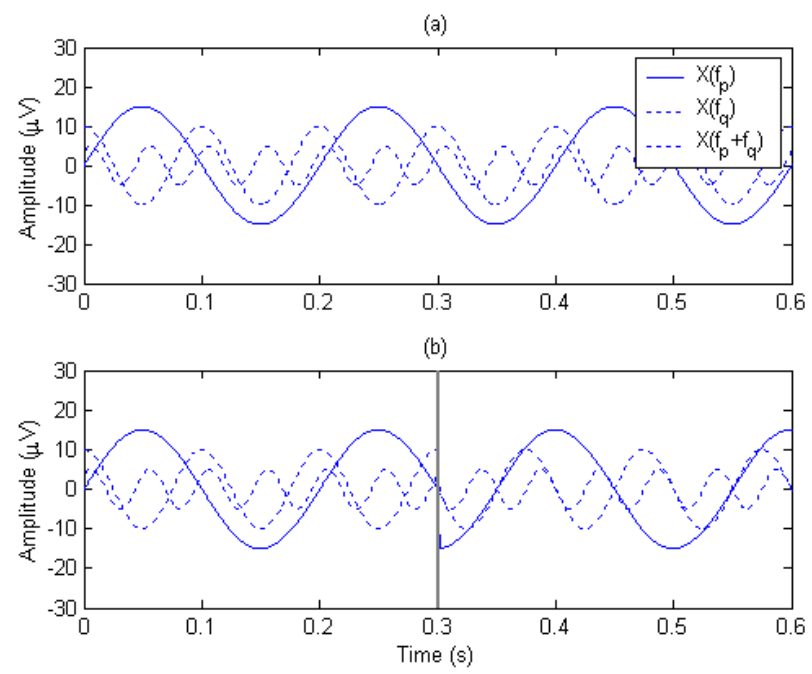

FIGURE 1. (a) Harmonics $X\left(f_{p}\right), X\left(f_{q}\right)$ and $X\left(f_{p}+f_{q}\right)$ maintain a constant phase relationship in time and $(b)$ the phases of the three harmonics vary independently in time as indicated by a different phase relationship for the time intervals (epochs) either side of the vertical line. 
One of the most established applications of EEG-based bispectral analysis involves the determination of an index for depth of brain anaesthesia in the form of the bispectral index, which is extensively documented [23,26,30,36-39]. However, bispectral analysis has also been successfully and widely applied as a diagnostic tool within studies involving: phases of epileptic seizure [40,41]; identification of epileptic and focal cerebral ischemia [40,42]; sleep detection [43]; evaluation of the degree of brain maturation in neonates [44]; differentiation of quadriceps muscle activation [45]; and normal sinus versus ischemic, tachycardia and fibrillation cardiac rhythms [31,46].

Example bicoherence spectra for asymptomatic, healthy adults in a somewhat neutral or largely non-stimulated neural state are seemingly unremarkable with example spectra respectively shown in figures $2 a$ and $2 b$ for EEG [34] and electromyogram (EMG) [47]. A further EEG example for higher alpha activation is given in figure 2c, with figure $2 \mathrm{~d}$ showing its corresponding harmonicamplitude-reflecting bispectrum [48].

The characteristic triangular shape of bicoherence spectra is a consequence of the mathematical permutations of $f_{p}, f_{q}$ and $f_{p+q}$ that are possible without exceeding the maximum spectral frequency. Chaotic-like EEG- and EMG-based spectra such as those of figures $2 \mathrm{a}$ and $2 \mathrm{~b}$ contain characteristic parallel ridge-like contours of high $\operatorname{bic}\left(f_{p}, f_{q}\right)$ at approximately $45^{\circ}$ to the $x$-axis, as highlighted in those figures and which are also identifiable in figure 2c for higher neural activation. These contours are explicitly noted or identifiable within some of the earliest bicoherence studies [30,31,49] and are considered typical of a normal chaotic response [31,49] which is also observed within electrocardiogram (ECG) bicoherence spectra [31,50]. However, these contours can also be interpreted as several frequencies being coupled to the same $f_{p+q}$ in concert with some form of complex oscillatory $\operatorname{bic}\left(f_{p}, f_{q}\right)$ behaviour across all frequencies (this latter interpretation is particularly relevant for Subsection 1.3). Also indicated by spectra such as figure 2c are couplings across the standard EEG frequency bands (with such inter-band couplings known to be dependent on EEG state [32,48,51]), and higher-order spectral aspects (e.g., self-coupling of diagonal bicoherence contours [32]) have also been studied as addressed further within Subsections 1.3 and 3.3.5.

The chaotic-like nature of EEG bicoherence spectra as exemplified by figure 2a, when the CNS is busy maintaining homeostasis, arguably defies phase relationship expectations of a highly intelligent communication platform and provides motivation for new higher-order forms of phase analysis. Also adding to this motivation is the fact that, while bispectral analysis displays previously cited clinical sensitivities, it is subject to several numerical and physical limitations:

\section{Limitations of bispectral analysis}

- Bispectral equations are statistical in nature by their reliance on a vectorial cancellation addition process over all epochs [30,47], with perfect vectorial cancellation yielding $\operatorname{bic}\left(f_{p}, f_{q}\right)=0 \%$ unlikely to mathematically occur due to the finite number of epochs utilised, even if the phase relationships between $X\left(f_{p}\right), X\left(f_{q}\right)$ and $X\left(f_{p}+f_{q}\right)$ are in actuality completely random.

- The consideration of only second-order (or other relatively low-order) phase couplings is a mathematical simplification (arguably an over-simplification) of the complexity of brain neurophysiology. That is, the complex, nonlinear nature of the brain is expected to contain important higher-order, nonlinear processes, as recognised since the earliest bispectral studies [30,31]. Indeed, it is reasonable that many harmonics might instead be expected linked by dynamically changing and sophisticated phase relationships within the impulse encoding of the CNS.

- EEG harmonics oscillate up to $\approx 60$ times per second (since the maximum frequency of conventional EEG frequency spectra is $\approx 60 \mathrm{~Hz}$, but may even be higher). Over a given epoch, typically up to several seconds duration, bispectral analysis assumes that all $\phi_{n}$ remain constant. When harmonics are oscillating at up to $\approx 60 \mathrm{~Hz}$, the assumption that their $\phi_{n}$ remain constant over a several, or even partial, second epoch duration is tenuous. The trend towards using increasingly (in some cases unnecessarily) higher sampling frequencies for EEG acquisition does not overcome this limitation.

- Like the more fundamental EEG indices summarised within this Subsection, while bispectral analysis provides useful clinical information, due to its physical nature the revelations it provides in relation to CNS impulse encoding is and can only be limited.

\subsection{HIGHER-ORDER PHASE ANALYSIS OF THE PRESENT STUDY}

As noted above, the existence of high-order EEG aspects have been acknowledged within scientific literature for decades, with high-order analysis techniques applied to phase couplings, synchronous relationships across frequencies, and autoregressive EEG modelling formalisms [29,32,42,48,51-54]. As a specific example, in a majority of EEG burst patterns that display high values along the bicoherence diagonal, up to tenth-order (and most commonly fifth-order) self-frequency and self-phase couplings have been identified and analysed [32]. 
(a)

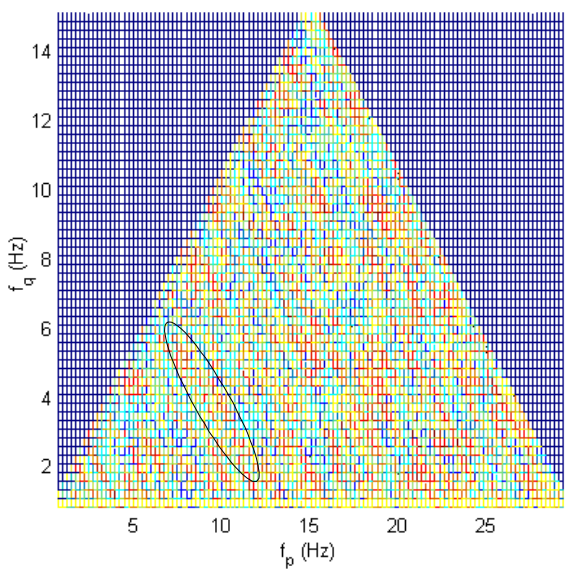

(c)

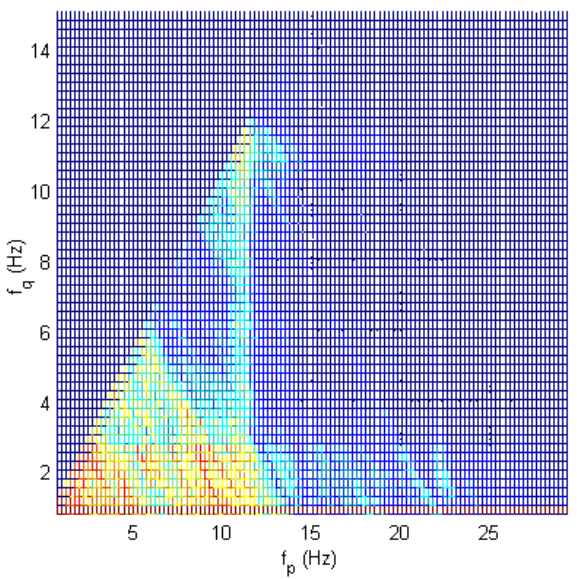

$\operatorname{bic}\left(f_{p}, f_{q}\right)$

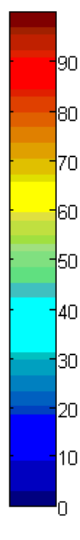

$\operatorname{bic}\left(f_{p}, f_{q}\right)$

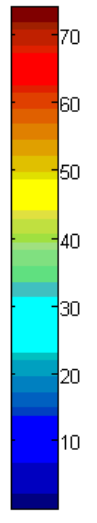

(b)

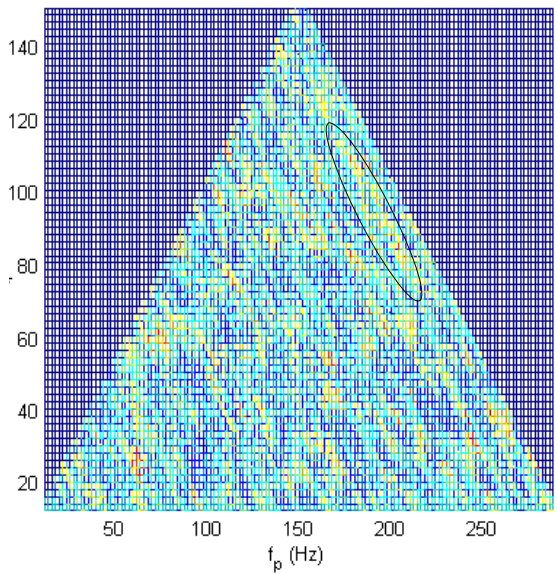

$\operatorname{bic}\left(f_{p}, f_{q}\right)$

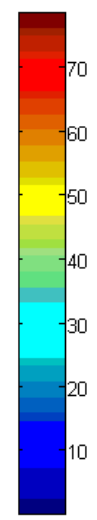

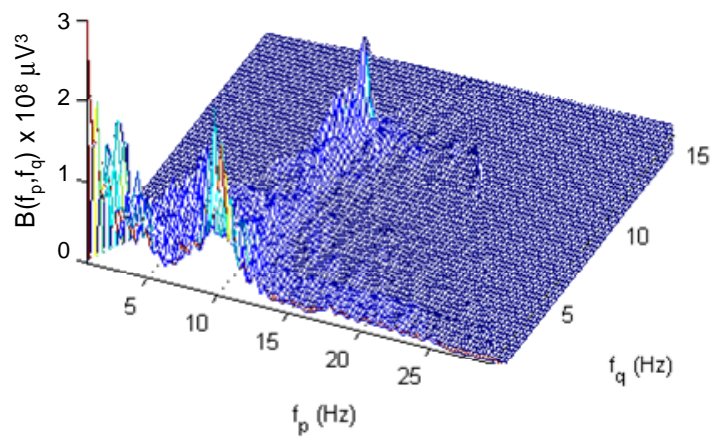

FIGURE 2. Typical non-stimulated, asymptomatic chaotic-like bicoherence spectrum for (a) EEG [34] and (b) EMG [47] which display bic( $f_{p}$, $\left.f_{q}\right)$ calculated for all $f_{p}$ and $f_{q}$, with typical ridge-like contours highlighted. An example EEG bicoherence spectrum for higher alpha activation is given in (c), with (d) showing its corresponding bispectrum [48]. bic $\left(f_{p}, f_{p}\right)=100 \%$ indicates a constant phase relationship between the harmonics $X\left(f_{p}\right), X\left(f_{a}\right)$ and $X\left(f_{p+q}\right)$, whereas bic $\left(f_{p}, f_{q}\right)=0 \%$ indicates that the phases of the harmonics vary independently.

The somewhat periodic bicoherence contours across frequencies noted in Subsection 1.2 are also indicative of some higher-order phase interrelationship, with this interrelationship including an oscillatory component. Given this component and the range of other oscillatory behaviours that are inherent within the complexities of the $\mathrm{EEG}^{1}$, the suggestion of some oscillatory aspect to a new form of high-order EEG phase analysis is reasonable.

\footnotetext{
${ }^{1}$ Note that EEG oscillations of various forms are well known (e.g., within EEG burst segments [32]), and any prominent spectral peak (i.e., alpha or other) within the conventional EEG frequency spectrum must mathematically
} trace to an oscillatory component of the original time-domain waveform.
Further contributing to this reasonability, it may be said that the electrophysiological ion transport origin of the EEG (i.e., the action potential) and above oscillatory phase coupling behaviour across spectral frequencies as indicated by periodic bicoherence contours, are together broadly consistent with other findings involving oscillatory behaviour within the area of ion transport and cellular communication (e.g., broad consistency with the findings of a calcium signalling study [55]). That study demonstrates how $\mathrm{Ca}^{2+}$ ions regulate a wide spectrum of cellular processes and examines the frequency encoding of cytosolic $\mathrm{Ca}^{2+}$ concentration oscillations. The oscillatory $\mathrm{Ca}^{2+}$ concentration patterns display large spatial and 
temporal diversity with suggestions of both amplitude and frequency modulation akin to conventional radio broadcast communication, and the oscillatory behaviour originates from an intricate concert action between several $\mathrm{Ca}^{2+}$ transporters at the cellular level [55].

Thus, the potential for some manner of deliberately structured oscillations across the frequency spectrum of the EEG, and more broadly across cellular communication processes, provides a justified basis for the application of a new form of high-order analysis, whereby a Fourier (or similar) transformation is applied to conventional EEG frequency spectra. That is, the study's commencing modus operandi requires a Fourier transformation to be twice applied to time-domain EEG data. From this point forward such double application is assigned the notation, $F T^{\prime}$, with the physical significance and implications of $F T^{\prime}$ in terms of CNS impulse encoding presented throughout.

\section{THEORY}

As justified above, the present study is founded upon a fast Fourier transformation (FFT) being twice applied to timedomain EEG data and assigned the notation, $F T^{\prime}$, with the second FFT applied to the AC components of the EEG's conventional amplitude versus frequency spectrum. For an $\mathrm{N}$-point EEG acquired with a sampling frequency and time of $f_{s}$ and $T$ respectively, standard FFT analysis yields a maximum spectral frequency of $f_{\max }=f_{s} / 2$ and a frequency resolution of $\Delta f=f_{s} / N$. $F T^{\prime}$ in turn reverts to a time-domain with a maximum of $T_{\max }^{\prime}=T / 2$ and resolution of $\Delta T^{\prime}=2 / f_{s}$. This resolution of the (N/4-point) $F T^{\prime}$ spectrum is more coarse than the original time-domain's resolution for which $\Delta T=1 / f_{s}=T / N$ as is standard.

The physical interpretation of $F T^{\prime}$ may be approached from original time-domain or literal transformation viewpoints, though both viewpoints ultimately have the same mathematical origin. The first-mentioned viewpoint considers the time-domain characteristics of the EEG that lead to $F T^{\prime}$ characteristics. This viewpoint is complex since the $F T^{\prime}$ does not simply return a waveform to its original time-domain form and different time-domain waveforms may result in the same $F T^{\prime}$ spectrum (hence the use of "effective time" as a domain descriptor from this point). As an example of this latter uniqueness issue, two simple test waveforms that are symmetrical, whereby amplitude or phase symmetrically steps up versus steps down in time, will have the same $F T^{\prime}$ spectrum. While the $F T^{\prime}$ spectrum is naturally reflective of the degree to which the original time-domain fluctuates, visualisation of how the original time-domain waveform influences the final $F T^{\prime}$ is representative of a substantial study in its own right.

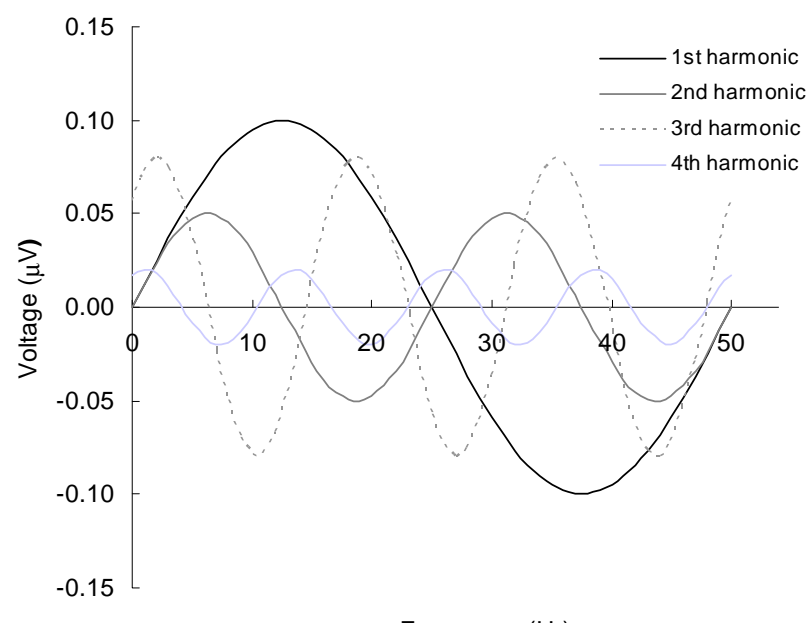

Frequency $(\mathrm{Hz})$

FIGURE 3. Representation of the physical decomposition of a conventional frequency spectrum resulting from a double Fourier transformation, or $F T^{\prime}$, process.

The more easily visualised $F T^{\prime}$ viewpoint or physical interpretation simply deconstructs the conventional frequency spectrum of the FFT (or comparable algorithm) into sinusoidal harmonics (plus standard DC offset term) that are functions of frequency rather than time, with an additional oscillation occurring across the conventional frequency spectrum with each successive harmonic, as per figure 3 showing the first four harmonics (representative only) of $F T^{\prime}$ deconstruction. It follows, and is visually apparent, from figure 3 that the amplitude of any given conventional FFT harmonic (i.e., the amplitude of the conventional FFT spectrum at a particular frequency) is constructed from the sum of all $F T^{\prime}$ harmonics at that chosen frequency. Due to the now sinusoidal linkage of conventional FFT amplitudes and allowed phase movements between the $F T^{\prime}$ harmonics, such an acrossfrequency depiction results in high degrees-of-freedom and frequency couplings far beyond second-order.

The $F T^{\prime}$ spectrum is insensitive to phase constancy in the original time-domain, which is consistent with its derivation from the FFT amplitude (rather than phase) spectrum. That is, spectral characteristics of the $F T^{\prime}$ will not be attributable to the constant phases of the conventional FFT harmonics. However, the FT' spectrum is sensitive to changing phases (modulatory or otherwise) within the constituents of the original time-domain waveform, which are expected to occur in practice. For example, a simple modulation in phase of a conventional harmonic will introduce an oscillation within the $F T^{\prime}$, with the magnitude of the $F T^{\prime}$ oscillation being proportional to the magnitude of the original modulation. This manner of phase sensitivity potentially negates the need for division of the original time-domain waveform into epochs, which is a limiting requirement of some existing phase-based analysis techniques as per Subsection 1.2. 


\section{METHOD}

\subsection{EEG DATA FOR ANALYSIS}

Two EEG data sets were constructed from independent sources so as to represent several clinically dissimilar scenarios and provide opportunity for technique validation and comparison across diverse neurological states. Data set construction also covered relatively short and long $T$ values with the intention of capturing high-order phase relationships that might occur over relatively short and long time scales whilst also defining relatively fine $\Delta f$ values (values of all signal processing parameter combinations are specified in Subsection 3.2). The data sets pertaining to nine adult subjects are described as follows:

\subsubsection{EEG DATA SET 1 (SHORT T)}

The first EEG data set pertains to two adult subjects, one classified as young and healthy, and the other as elderly with a probable Alzheimer's disease diagnosis. Data for these two subjects were collected by [56] in both eyes-open and eyes-closed states for all standard 19 channels of the international 10-20 referencing system of electrode placement [57], with all channels simultaneously sampled at $f_{s}=128 \mathrm{~Hz}$ for $8 \mathrm{~s}$. Utilisation of this data within the present study focused on prefrontal (Fp1) and occipital (O2) electrode placements as specified throughout the continuing methodology and results to follow.

\subsubsection{EEG DATA SET 2 (LONG T)}

The second EEG data set pertains to seven healthy adult subjects undergoing clinical sleep (full overnight polysomnographic) studies and, for cross-sectional validation purposes, represented a compilation from sources that included [58] and [59] which are accessible via, and detailed within, the well-established PhysioNet database [60]. Note that while [58] and [59] include symptomatic subjects, only control subjects with no pathology were selected for the compilation (e.g., a subject chosen from the sleep apnoea study of [59] had an apnoea-hypopnoea index, or AHI, of $<5$ and was therefore considered normal).

Complied data had been continuously scored for sleep stage over contiguous $30 \mathrm{~s}$ intervals whereby each interval was assigned either: awake, stages 1 to 4 (4 being the deepest stage), or rapid-eye-movement. Data from a wide range of conventional polysomnographic electrode placements are available within the compilation sources; those utilised by the preset study predominantly focused on central (C3 or C4) and occipital (O2) electrode placements, with further specification given as results are presented. A wide range of $f_{s}$ values are similarly available within the sources and see Subsection 3.2 for comments on the seemingly low, but deliberate strategy behind, chosen $f_{s}$ values that ranged from 100 to $200 \mathrm{~Hz}$.

\subsection{HIGH RESOLUTION FFT OF DATA SETS}

An FFT was applied (using National Instruments Labview 2016 full development system software) to Data Sets 1 and 2 so as to achieve high resolution frequency spectra of $\Delta f=0.125$ and $0.005 \mathrm{~Hz}$ respectively, in-turn equating to respective signal processing values of $N=1024, T=8$ (Data Set 1 ) and $N=20,000$ to $40,000, T=200 \mathrm{~s}$ (Data Set 2). Given the extended (overnight) duration of the EEG waveforms within Data Set 2, the 20,000- to 40,000-point FFTs based on $T=200 \mathrm{~s}$ are representative of select segments of any given EEG. These segments of the total EEG were chosen to allow comparison across a range of sleep stages. While not a key comparison index of the present study, SEF with a 95\% cut-off was routinely calculated as a general assessment indicator for all frequency spectra.

The seemingly low $f_{s}=100 \mathrm{~Hz}$ value utilised within Data Set 2 related to stage 4 sleep EEG segments for one subject only. For these EEG segments, SEF was less than $30 \mathrm{~Hz}$ and so the appropriateness of $f_{s}=100 \mathrm{~Hz}$ is maintained in accordance with the Nyquist sampling theorem. The maximum SEF in all other cases was less than (most often appreciably) $55 \mathrm{~Hz}$, with remaining $f_{s}$ values comfortably meting corresponding Nyquist requirements. The discipline's trend towards relatively higher $f_{s}$ (e.g., $f_{s}>250$ $\mathrm{Hz}$ ), alluded to within Subsection 1.2, was not followed to avoid or minimise the computation of FFT harmonics at impractically high $f_{\max }$ that would otherwise result from the relatively long $T$ (and fine $\Delta f$ ) values utilised.

\subsection{FT'ANALYSIS OF DATA SETS}

\subsubsection{SECOND FFT APPLICATION}

An FFT was applied to all AC components of conventional amplitude versus frequency spectra of Subsection 3.2 to yield corresponding $F T^{\prime}$ amplitude and phase spectra, in accordance with the prior Theory Section. For Data Set 1, $F T^{\prime}$ spectra from electrode channels 1 to 12 (prefrontal, frontal, central, temporal T3/T4) and from channels 13 to 19 (occipital, parietal, temporal T5/T6) were also combined to give average $F T^{\prime}$ spectra for these electrode groupings for both subjects and both eyes-open and closedstates. $F T^{\prime}$ phase profiles were shifted from a relative range of -180 to $+180^{\circ}$ (the FFT default) to a relative range of 0 to $+360^{\circ}$ to aid visualisation. As part of technique validation testing, the effect of windowing (Blackman, Hanning and Hamming) EEG data on $F T^{\prime}$ spectra was recorded for select EEG segments of Data Sets 1 and 2.

\subsubsection{PHASE ANALYSIS OF FT' SPECTRA}

In addition to standard functional relationship analysis of warranting $F T^{\prime}$ spectra (e.g., linearity analysis of profiles for stage 4 sleep), continuing (deeper) phase-based analysis was applied to a selection of $F T^{\prime}$ spectra derived from both data sets. For Data Set 1, prefrontal and occipital eyesclosed EEG were chosen for this continuing analysis since 
such electrode placement combinations allow for useful, contrasting clinical bicoherence comparison [39] and occipital EEG with eyes-closed provides strong EEG and alpha signals (the posterior dominant rhythm, or PDR, is enhanced during relaxed wakefulness [4,61]). Strong alpha activity is a focus of the present study's cross-sectional comparison because: (i) literature now links alpha activity to not just relaxed consciousness and passive attention, but additionally to active roles in neural network communication and co-ordination including short-term memory and working memory retention [51], and (ii) conventional spectral peaks might conceivably be linked to quantum mechanical resonances (see Evidence of a Quantum Nature Subsection 5.5). Accordingly, FT' spectra derived from Data Set 2 and selected for this continuing phase analysis also displayed conventional spectral peaks (including sigma sleep spindle) for states from relaxed consciousness to stages 1 to 3 sleep.

The continuing phase analysis of $F T^{\prime}$ spectra was based upon discrete phase transitions, $\Delta \phi$, observed within many non-stage 4 sleep spectra (from this point $\phi$, representative of conventional harmonic phase, dually represents $F T^{\prime}$ harmonic phase). These $\Delta \phi$ are described throughout as "upward" when the $F T^{\prime}$ phase profile markedly steps up (from some local minimum to an adjacent local maximum) as a function of effective time. Conversely, $\Delta \phi$ are described throughout as "downward" when the $F T^{\prime}$ phase profile vertically steps down (from a local maximum to an adjacent minimum) as a function of effective time, with the final descriptor of "horizontal" applying to $\Delta \phi$ between local maxima of $F T^{\prime}$ phase profiles. Analysis involved the construction of a polar plot (phase wheel) of $\Delta \phi$ values for each $F T^{\prime}$ phase profile (with $\Delta \phi$ values referred to as radials within phase wheels). Any radial alignment to within $\pm 0.5^{\circ}$ of conventional landmark trigonometric angles (e.g., $30^{\circ}$, $45^{\circ}, 60^{\circ}, 90^{\circ}$, and counterparts within quadrants two to four), referred to here as primary radials, was indicated within phase wheels. The study's design initially applied broad defining criteria as to what constitutes a primary radial (which initially also included integer and common fraction multiples of $20^{\circ}$ not already specified), but progressively applied self-refinement of these defining criteria in accordance with the remaining Method Subsections. Hence, the term potential primary radial is initially used within the Results Section.

Within phase wheels, the ratios of sector angles (for sectors formed between radials and other radials or primary radials) that align to within $1.0 \%$ of some common fraction were also identified. Such alignment testing represented a rudimentary but necessary initial aspect of a deeper phasebased analysis for upward $\Delta \phi$, the full description of which continues within the following Subsections.

\subsubsection{CHI-SQUARED STATISTICAL ANALYSIS OF $\Delta \phi$ RADIALS}

A frequency of occurrence distribution based upon all upward $\Delta \phi$ from seven (cross-sectionally representative) phase wheels was generated with a distribution resolution of $\delta \phi= \pm 0.5^{\circ}$ applied. A second frequency of occurrence distribution was generated with transitions from 180 to $360^{\circ}$ converted to complementary angles from 0 to $180^{\circ}$ (e.g., $355^{\circ} \equiv 5^{\circ}$ and so on), with conversion rationale given within the Discussion. Respective null hypotheses of expected normal $\Delta \phi$ distributions from 0 to $360^{\circ}$ and 0 to $180^{\circ}$ were tested via standard chi-squared analysis with an applied significance threshold of $p<0.001$. For any unexpected distribution, polar angles about which $\Delta \phi$ values clustered were recorded for further analysis.

\subsubsection{BINOMIAL DISTRIBUTION STATISTICAL ANALYSIS OF $\triangle \phi$ RADIALS}

For individual and combinations of phase wheels, binomial distribution analysis gave the probability, $P$, of $\Delta \phi$ values aligning with predetermined primary radials, with predetermination based upon both the prior-stated landmark polar angles and any radial angle about which an unexpected clustering of $\Delta \phi$ values may have occurred, as identified by the above chi-squared analysis. The $P$ of alignment of $h$ (from a total of $H$ ) radials with any of $m$ primary radials, to within a resolution of $\pm \delta \phi$ radians, for individual or combinations of phase wheels, is given from binomial probability theory [62] by (1):

$$
P={ }_{h}^{H} C\left(\frac{m \delta \phi}{\pi}\right)^{h}\left(1-\frac{m \delta \phi}{\pi}\right)^{H-h}
$$

\subsubsection{ITERATIVELY LINEARIZED FAMILY ANALYSIS OF $\triangle \phi$ RADIALS}

Based on the frequency of occurrence distribution applied to all upward $\Delta \phi$ radials from the seven phase wheels to which chi-squared analysis was applied, any irregular clusterings of $\Delta \phi$ about particular polar angles were said to form potential "Families" about these polar angles. For each potential Family, the separations between intra-Family radials were calculated and written in terms of quarter- or third-integer $(k=j / 4$ or $j / 3, j=1,2,3 \ldots)$ amounts of some to-be-determined proposed quantum increment value, $\alpha$, such that separation is given by $k \alpha$ (note that the $\alpha$ notation is independent of the "alpha" activity descriptor of conventional EEG frequency spectra). The $\alpha$ value for each established Family was extracted as the gradient of a (zero $y$-intercept) graph of separation versus $k$ multiplier in an iterative manner. The iterative linearization process involved a separation acceptance/rejection threshold of $1.0 \%$ difference between the actual separation and separation given by $k \alpha$. That is, $\alpha$ was recalculated upon each iteration until only separations with residuals of less than $1.0 \%$ were included within the linear regression (that applied $\quad r>0.9999, \quad p<0.001 \quad$ Family acceptance 
thresholds) used for the determination of $\alpha$, with a 95\% confidence interval (CI) that was established via application of a $t$-distribution to the gradient's standard error.

The original iterative method above may be seen to have parallel with the recursive method [32] (adapted from that of [53]) of accepting/rejecting elements contributing to $k$ thorder self-phase coupling (where the phase of an element is $k$ times the phase of another) within bicoherence indices. However, while such precedents exists for an expectation of phase multiples within high-order self-coupling, here the described $k \alpha$ iterative approach is based on the unique quantisation postulate that harmonics of increasing order as depicted by the physical deconstruction of figure 3 may potentially be separated by quantised phase increments which play a role within some to-be-determined form of discrete phase-shift keying (PSK) communication encoding [2,63].

Finally, the existence of any functional relationship between identified $\alpha$ values across all Families was investigated. Because individual Family $\alpha$ values may be readily scaled (e.g., $\alpha^{\prime}=2 \alpha$ say) with the required $1 / 4$ or $1 / 3$ transition separation multiplicity condition still maintained, relationship identification is potentially straight forward. Thus, any $\alpha$ scaling and relationship finalisation was based on correlation maximisation (the regression for which propagated $\alpha$ error via a Monte Carlo approach that easily achieved model parameter convergence to required confidence levels via 800 simulation iterations) along with $\alpha$ scaling reasonability such that the smallest separations ${ }^{2}$ within each Family corresponded to low $k$ values (e.g., 1/4, $1 / 2$ and 1 ). This finalisation in turn allowed for factors such as the overall occupancy of Families, and the extent to which these occupancies involved $k=j / 2$ separation multiples (only) of $\alpha$, to be considered for the potential classification of Families (e.g., as primary or secondary following an analogous quantum classification system), and indeed whether a certain type of $\alpha$ multiplicity is preferred within Families. Note that $k=j / 2$ multiples are of particular interest because of the fundamental commonality of full- and half-integer augmentations across many and various quantum systems.

\section{RESULTS}

This Section, for which conventional FFT and corresponding $F T^{\prime}$ spectral outcomes are generated from 31 example EEG segments pertaining to nine different subjects, is structured into two levels of analysis, Subsections 4.1 and 4.2. Within the latter Subsection, which contains the present study's foremost findings (with appropriate levels of statistical confidence), all $F T^{\prime}$ phase profiles that display characteristic discrete upward $\Delta \phi$ are combined within an overall analysis that provides insight into the mathematical structure of CNS

${ }^{2}$ This $\alpha$ scaling reasonability for the smallest separations included those outside of the $1.0 \%$ alignment threshold, if the two $\Delta \phi$ radials of any such separation were individually involved in other separations that met the $1.0 \%$ threshold. signal encoding for a broad spectrum of neural states. Other than the revelation of such insight, the present study is nonclinical, aiming to mathematically introduce and demonstrate the physical efficacy of the new EEG analysis technique to pave the way for future clinical studies. Hence, the snapshot spectral comparisons that follow within Subsection 4.1 are not for the purpose of clinical differentiation or diagnosis. To assist mathematical visualisation, spectra and associated analyses involving phase are displayed throughout in units of degrees, though dual radian equivalence is adopted in select cases.

\subsection{LEVEL 1 ANALYSIS: EXAMPLES AND OVERVIEW OF FT'SPECTRAL CHARACTERISTICS}

Example $F T^{\prime}$ spectra presented and compared within this Subsection contain harmonic amplitude and phase profiles in the same graph (i.e., such graphs contain dual $y$-axes).

\subsubsection{CROSS-SECTION OF FT' EXAMPLES FOR T = $8 \mathrm{~s}$ DURATION EEG SEGMENTS (DATA SET 1)}

An example alpha-active conventional FFT spectrum and its corresponding $F T^{\prime}$ spectrum, for occipital (O2) electrode placement upon the healthy subject with eyes-closed of Data Set 1, are respectively shown in figures $4 \mathrm{a}$ and $4 \mathrm{~b}$. The alpha peak accounts for approximately $28 \%$ of the spectrum integral in figure $4 \mathrm{a}$. For comparison, figures $4 \mathrm{c}$ and 4d respectively show conventional FFT and corresponding $F T^{\prime}$ of reduced alpha activity simultaneously collected from the same subject for prefrontal (Fp1) electrode placement. Select upward $\Delta \phi$ of figure $4 \mathrm{~b}$ are annotated with lower-case alphabetical labels, while select local maxima are annotated with a degrees label given by shifting the relative phase scale by a further $-100.7^{\circ}$ and rounding to the nearest integer (the actual shifted phase values of local maxima thus equal their corresponding rounded labels to within $\pm 0.5^{\circ}$ ). The values of all labeled quantities and their quantified alignment with landmark polar angles are given in table A1 of Appendix A.

Note that while $F T^{\prime}$ spectra extend beyond $t^{\prime}=1.0 \mathrm{~s}$, the $F T^{\prime}$ amplitude profiles generally decay to some relatively low baseline (albeit fluctuating) by this effective time. Hence, this initial $1.0 \mathrm{~s}$ decay period of $F T^{\prime}$ spectra is a focus for presented results and later discussion. Similar comparative outcomes (not graphically shown) in terms of general overall $F T^{\prime}$ amplitude profile shape are obtained when averaging the $F T^{\prime}$ profiles of EEG channels 13 to 19 (see Subsection 3.3.1 for electrode placement descriptors) and comparing against the average of the $F T^{\prime}$ profiles for EEG channels 1 to 12 .

Figure 4e presents a phase wheel displaying all (not just selectively labeled) upward $\Delta \phi$ of Figure $4 \mathrm{~b}$ via solid radials (of any colour), and the corresponding table 1 respectively gives phase wheel sector ratio values that align to within $1.0 \%$ of some identified nominal common fraction. The percentage differences between actual and nominal ratio values are bracketed within the central 
column of table 1 , with ratios falling outside $1.0 \%$ alignment but still worthy of qualitative note shown in light shaded text. Where a ratio involves a sector formed with a potential primary radial, that potential primary radial is noted within both table 1 (right-hand column) and figure 4e (as a dashed radial which in several instances is fully or partially overlapped by an actual $\Delta \phi$ radial - see table A1 for degree of overlap). Orange radial colouring reflects such overlapping with other colourings reflecting some connectedness in terms of sector formation. Also within figure 4e, dashed lines annotated as "mid" represent the polar location symmetrically between radial pairs. Table A2 gives select sector angle values for figure $4 \mathrm{e}$.
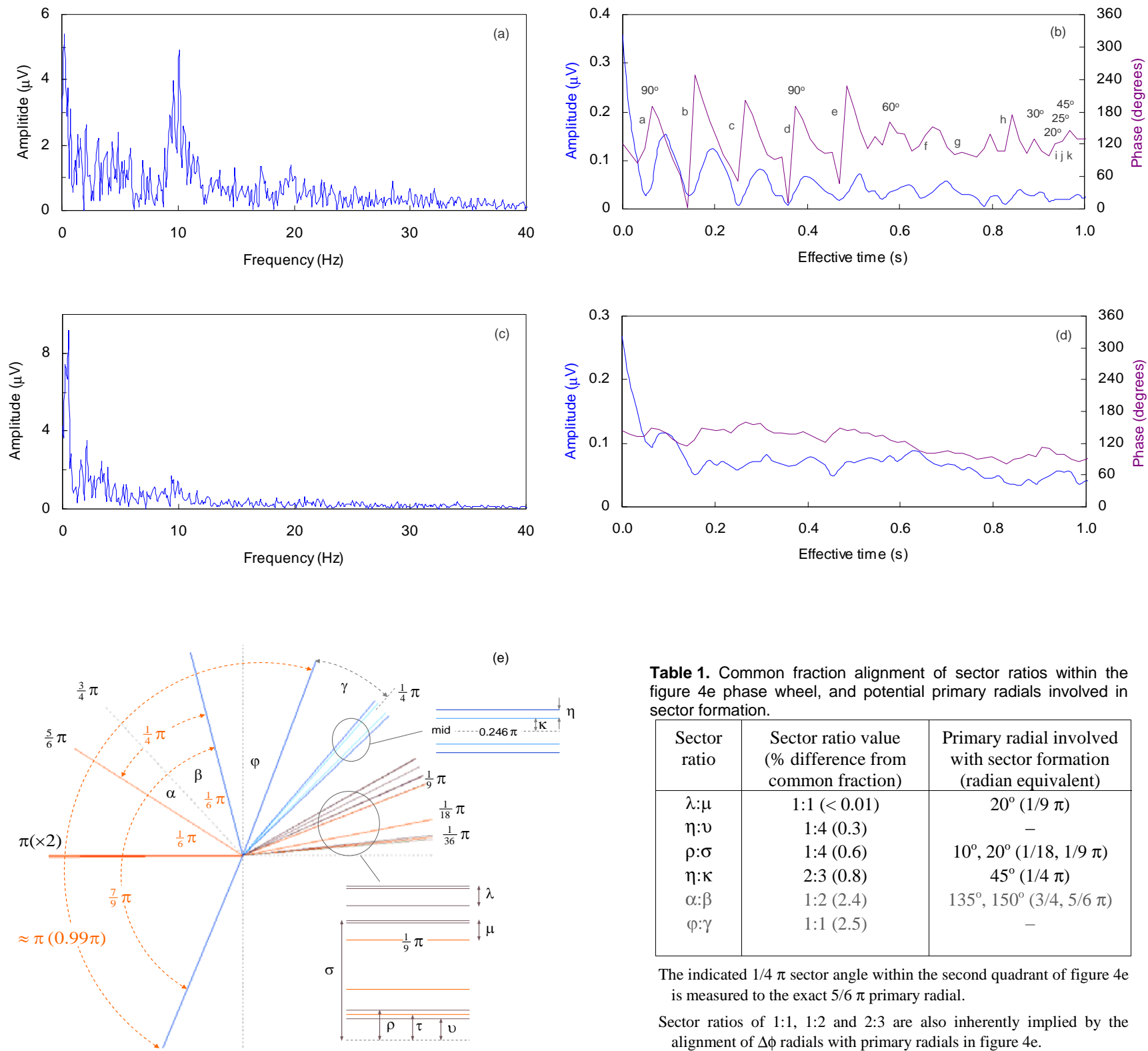

Table 1. Common fraction alignment of sector ratios within the figure $4 \mathrm{e}$ phase wheel, and potential primary radials involved in sector formation.

\begin{tabular}{|c|c|c|}
\hline $\begin{array}{c}\text { Sector } \\
\text { ratio }\end{array}$ & $\begin{array}{c}\text { Sector ratio value } \\
\text { (\% difference from } \\
\text { common fraction) }\end{array}$ & $\begin{array}{c}\text { Primary radial involved } \\
\text { with sector formation } \\
\text { (radian equivalent) }\end{array}$ \\
\hline$\lambda: \mu$ & $1: 1(<0.01)$ & $20^{\circ}(1 / 9 \pi)$ \\
$\eta: v$ & $1: 4(0.3)$ & - \\
$\rho: \sigma$ & $1: 4(0.6)$ & $10^{\circ}, 20^{\circ}(1 / 18,1 / 9 \pi)$ \\
$\eta: \kappa$ & $2: 3(0.8)$ & $45^{\circ}(1 / 4 \pi)$ \\
$\alpha: \beta$ & $1: 2(2.4)$ & $135^{\circ}, 150^{\circ}(3 / 4,5 / 6 \pi)$ \\
$\varphi: \gamma$ & $1: 1(2.5)$ & - \\
\hline
\end{tabular}

The indicated $1 / 4 \pi$ sector angle within the second quadrant of figure $4 \mathrm{e}$ is measured to the exact $5 / 6 \pi$ primary radial.

Sector ratios of 1:1, 1:2 and 2:3 are also inherently implied by the alignment of $\Delta \phi$ radials with primary radials in figure $4 \mathrm{e}$.

FIGURE 4. Conventional frequency spectra of a healthy adult subject with eyes-closed for (a) occipital (O2) with relatively high alpha activity and (c) prefrontal (Fp1) EEG. For each conventional frequency spectrum the corresponding (adjacent) double Fourier transformation of the EEG, or $F T^{\prime}$ spectrum, is respectively given in (b) and (d). Annotated local maxima labels in (b) are generated by adding $-100.7^{\circ}$ to the relative phase scale of the right vertical axis and rounding the resulting maxima values to the nearest integer. All upward $\Delta \phi$ of (b) are displayed as radials within the phase wheel of (e), with notable ratios of phase wheel sector angles given within the adjacent table 1 (sector angle symbolism is generic and separate to any elsewhere duplication). 
Figures 5a and 5b respectively show conventional FFT and corresponding $F T^{\prime}$ spectra for occipital (O2) electrode placement upon the probable Alzheimer's subject with eyes-closed of Data Set 1 , with figures $5 c$ and $5 d$ showing equivalent spectra for simultaneously collected prefrontal (Fp1) electrode positioning. Select local maxima in figures $5 \mathrm{~b}$ and 5d are annotated with degree labels given by respectively shifting their relative phase scales by $-178.2^{\circ}$ and $-154.8^{\circ}$, and then rounding to the nearest integer; the values of labeled quantities are again given in table A1.
Phase wheels following the conventions of figure 4e and corresponding to figures $5 \mathrm{~b}$ and $5 \mathrm{~d}$ are respectively given in figures $5 \mathrm{e}$ and $5 \mathrm{f}$ (note that results presented within Subsection 4.2 and the later Discussion Section refine and justify what constitutes a primary radial within such figures). Table 2 gives phase wheel sector ratio values that align to within $1.0 \%$ of some identified nominal common fraction, while table A2 gives select sector angle values for figures $5 e$ and $5 f$
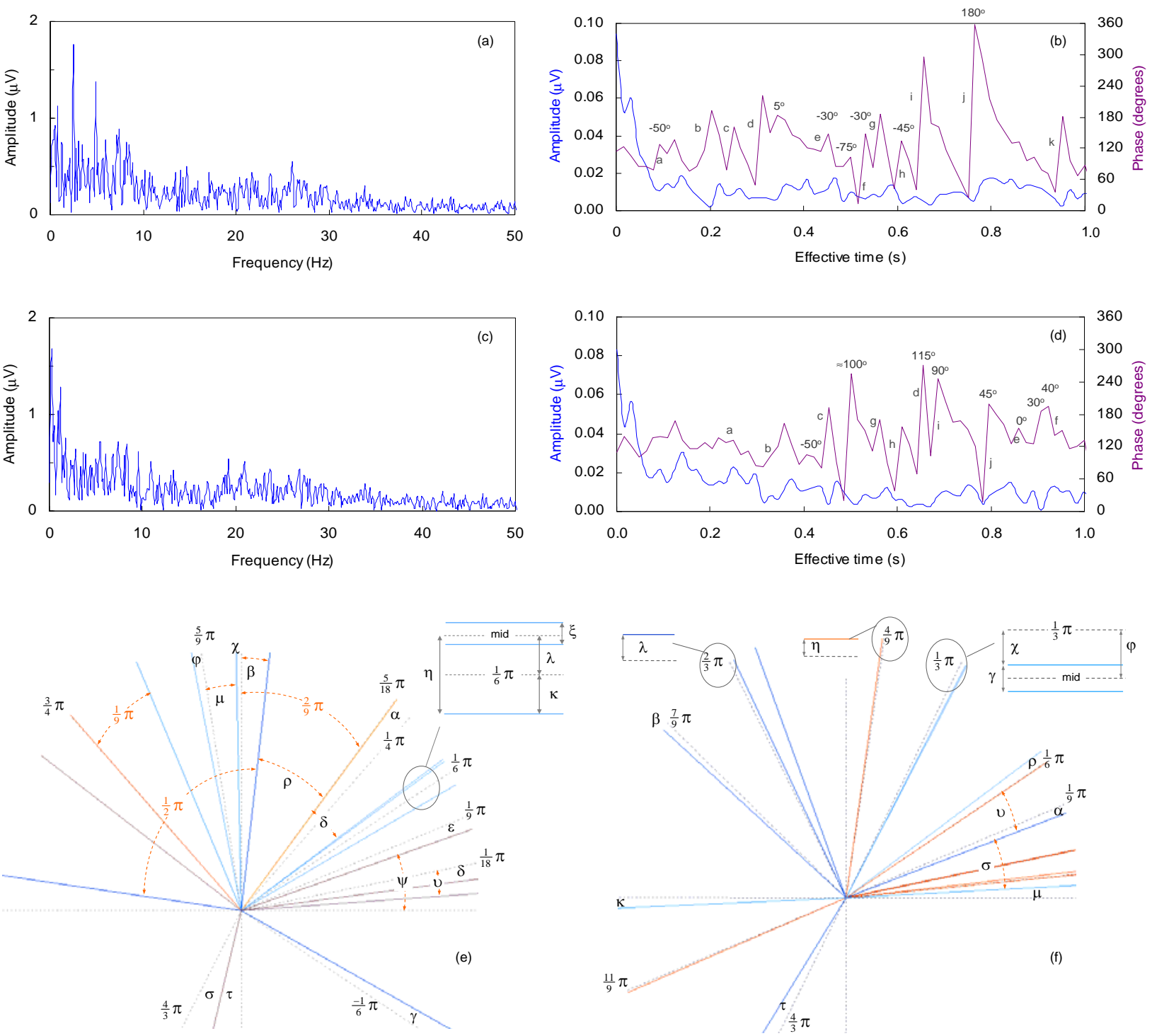

FIGURE 5. Conventional frequency spectra for elderly subject diagnosed with probable Alzheimer's disease with eyes-closed for (a) occipital (O2) and (c) prefrontal (Fp1) EEG. For each conventional frequency spectrum the corresponding (adjacent) double Fourier transformation of the EEG, or

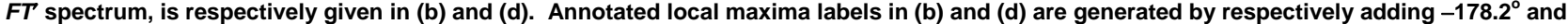
$-154.8^{\circ}$ to the relative phase scale of the right vertical axis and rounding the resulting maxima values to the nearest integer. All upward $\Delta \phi$ of (b) and (d) are respectively displayed as radials within the phase wheels of (e) and (f), with notable ratios of phase wheel sector angles given within table 2 (sector angle symbolism is generic and separate to any elsewhere duplication). 
Table 2. Common fraction alignment of sector ratios within figures $5 e$ and $5 f$, and potential primary radials involved in sector formation.

\begin{tabular}{|c|c|c|}
\hline $\begin{array}{l}\text { Sector } \\
\text { ratio }\end{array}$ & $\begin{array}{l}\text { Sector ratio value } \\
\text { (\% difference from } \\
\text { common fraction) }\end{array}$ & $\begin{array}{c}\text { Primary radial involved with } \\
\text { sector formation (radian } \\
\text { equivalent) }\end{array}$ \\
\hline \multicolumn{3}{|c|}{ figure $5 \mathrm{e}$} \\
\hline$\gamma: \beta$ & $1: 2(0.02)$ & $-30^{\circ}, 90^{\circ}(-1 / 6,1 / 2 \pi)$ \\
\hline$v: \psi$ & $3: 8(0.2)$ & $10^{\circ}, 360^{\circ}(1 / 18,2 \pi)$ \\
\hline$\chi: \varphi: \eta$ & $1: 2: 5(0.3)$ & $30^{\circ}, 90^{\circ}, 100^{\circ}(1 / 6,1 / 2,5 / 9 \pi)$ \\
\hline$\kappa: \lambda^{\#}$ & $1: 1(0.9)$ & $30^{\circ}(1 / 6 \pi)$ \\
\hline$\eta: \mu$ & 2:3 (0.9) & $30^{\circ}, 100^{\circ}(1 / 6,5 / 9 \pi)$ \\
\hline$\delta: \psi^{*}$ & $1: 5(<1.0)$ & $10^{\circ}, 50^{\circ}, 360^{\circ}(1 / 18,5 / 18,2 \pi)$ \\
\hline$\varepsilon: \lambda$ & $1: 1(1.2)$ & $20^{\circ}, 30^{\circ}(1 / 9,1 / 6 \pi)$ \\
\hline$\alpha: \beta^{*}$ & $2: 3(1.3)$ & $45^{\circ}, 50^{\circ}, 90^{\circ}(1 / 4,5 / 18,1 / 2 \pi)$ \\
\hline$\delta: \rho^{*}$ & $1: 1(2.0)$ & $50^{\circ}(5 / 18 \pi)$ \\
\hline$\sigma: \tau$ & $1: 1(2.0)$ & $300^{\circ}, 270^{\circ}(-1 / 3,3 / 2 \pi)$ \\
\hline \multicolumn{3}{|c|}{ figure $5 f$} \\
\hline$\varphi: \rho$ & $1: 2(0.04)$ & $30^{\circ}, 60^{\circ}(1 / 6,1 / 3 \pi)$ \\
\hline$\gamma: \eta$ & $2: 3(<0.10)$ & $60^{\circ}, 80^{\circ}(1 / 3,4 / 9 \pi)$ \\
\hline$v: \sigma$ & $3: 4(0.2)$ & $30^{\circ}(1 / 6 \pi)$ \\
\hline$\alpha: \tau$ & $1: 2(0.2)$ & $20^{\circ}, 240^{\circ}(1 / 9,4 / 3 \pi)$ \\
\hline$\rho^{* *}: \mu$ & 1:1(0.5) & $30^{\circ}, 360^{\circ}(1 / 6,2 \pi)$ \\
\hline$\varphi: \mu$ & $1: 2(0.6)$ & $60^{\circ}, 360^{\circ}(1 / 3,2 \pi)$ \\
\hline$\lambda: \beta$ & 2:3 (0.8) & $120^{\circ}, 140^{\circ}(2 / 3,7 / 9 \pi)$ \\
\hline$\chi: \kappa$ & 1:2 (1.4) & $60^{\circ}, 180^{\circ}(1 / 3,1 \pi)$ \\
\hline$\alpha: \beta$ & 1:1 (1.6) & $20^{\circ}, 140^{\circ}(1 / 9,7 / 9 \pi)$ \\
\hline$\eta: \varphi$ & $2: 5(1.6)$ & $60^{\circ}, 80^{\circ}(1 / 3,4 / 9 \pi)$ \\
\hline
\end{tabular}

\#Angles $\kappa$ and $\lambda$ are measured about the exact $1 / 6 \pi$ primary radial in figure $5 \mathrm{e}$.

${ }^{*}$ Angles $\rho, \delta$ and $\alpha$ are measured to the $\Delta \phi$ radial that aligns near the exact 5/18 $\pi$ primary radial in figure $5 \mathrm{e}$.

Sector ratios of 1:2, 4:9 and 2:9 are also inherently implied by the alignment of $\Delta \phi$ radials with primary radials in figure $5 \mathrm{e}$.

${ }^{* *}$ Angle $\rho$ is measured to the exact $1 / 6 \pi$ primary radial in figure $5 f$.

Sector ratios of 1:2, 1:3, 1:4, 1:6 and 3:4 are also inherently implied by the alignment of $\Delta \phi$ radials with primary radials in figure $5 f$.

\subsubsection{CROSS-SECTION OF FT' EXAMPLES FOR}

$T=200$ s DURATION EEG SEGMENTS (DATA SET 2)

Note that for this Subsection the hash numbering of subjects within figures is consistent with anonymous numerical identifiers used by the original cited data sources that compile (overnight polysomnographic) Data Set 2. Figures $6 \mathrm{a}$ and $6 \mathrm{~b}$ respectively show example conventional FFT and corresponding $F T^{\prime}$ spectra for an occipital (O2) EEG segment for which the subject is in a state generally described as possessing both awake and significant stage 1 sleep components (with the multi-stage description indicative of sleep scoring at $30 \mathrm{~s}$ intervals within the relatively long $T=200 \mathrm{~s}$ EEG segment). An oscillatory $\phi$ profile with approximate linear decay, as exemplified by figure $6 \mathrm{~b}$, is a common characteristic of sleep $F T^{\prime}$ phase profiles, especially when conventional FFT peaks (e.g., alpha, sigma sleep spindle) are present, in which case the amplitude of $\phi$ oscillation is generally indicative of peak dominance and decreases with increasing sleep depth. As per Subsection 4.1.1, upward $\Delta \phi$ of oscillations within spectra such as figure $6 \mathrm{~b}$ are more formally analysed within
Subsection 4.2. For later comparison purposes, the gradient of the phase profile in figure $7 \mathrm{~b}$, with error margin at $95 \%$ $\mathrm{CI}$, is $d \phi / \mathrm{d} t^{\prime}=-141 \pm 6^{0} \cdot \mathrm{s}^{-1}$ based on linear regression modelling $(r=-0.987, p<0.001)$.

Figures $6 \mathrm{c}$ and $6 \mathrm{~d}$ respectively show example conventional FFT and corresponding $F T^{\prime}$ spectra for a central (C4) EEG segment for a subject in stage 4 sleep. A model trend line based on linear regression of the phase profile in figure $6 \mathrm{~d}$, over a selected domain of interest $\left(0.1 \leq t^{\prime} \leq 0.7 \mathrm{~s}\right)$, is given in figure 6e stacked alongside similarly derived trend lines for five other $T=200 \mathrm{~s}$ stage 4 sleep segments of the same overnight EEG recording (hence the six consecutive segments, each separated by $10 \mathrm{~s}$, represent a total stage 4 sleep analysis time of $20.8 \mathrm{~min}$ for the same subject). Stacking is achieved by progressively (chronologically) vertically shifting each successive series by an additional $40^{\circ}$. Comparison figure $6 \mathrm{f}$ is derived in a similar manner for another subject in extended stage 4 sleep and for the same electrode placement. Note that domain commencement at $t^{\prime}=0.1 \mathrm{~s}$ in figures $6 e$ and $6 \mathrm{f}$ is due to a slight deviation from linearity over the domain $0.0 \leq t^{\prime} \leq 0.1 \mathrm{~s}$ for some such phase profiles (not apparent within figure 6d), and so the domain $0.1 \leq t^{\prime} \leq 0.7 \mathrm{~s}$ is maintained to allow consistent comparison across figures. Also, $y$-error bars in figures 7e and $7 \mathrm{f}$ are smaller than the graphical markers utilised and not resolvable by the figures' scales.

Annotated in figures 6e and $6 \mathrm{f}$ are $95 \% \mathrm{CI} d \phi / \mathrm{d} t^{\prime}$ values and linear regression statistics $(-r \geq 0.990, p<0.001$ in all cases). Within these figures, $d \phi / \mathrm{d} t^{\prime}$ ranges from $-163 \pm 9$ to $-203 \pm 7^{0} \cdot \mathrm{s}^{-1}(3.3 \%)$ and $-166 \pm 6$ to $-206 \pm 4^{\mathrm{o}} \cdot \mathrm{s}^{-1}(3.7 \%)$ respectively, with bracketed values giving the average of all intra-figure error margins when the margins are expressed as a percentage. A third similar stage 4 sleep analysis example (not graphically shown) gives a $d \phi / \mathrm{d} t^{\prime}$ range of $-190 \pm 5$ to $-236 \pm 8^{\circ} \cdot \mathrm{s}^{-1}(2.5 \%)$.

Figure $6 \mathrm{~g}$ focuses upon the first of the phase profiles in figure $6 \mathrm{e}$ and is therefore associated with the commencement of the extended 20.8 min stage 4 sleep analysis. This focused-upon profile demonstrates sight oscillatory behaviour, possibly attributable to some residual (less-deep) sleep component, and is partially mirrored by figure 6g's additional phase profile simultaneously collected for prefrontal (Fp2-F4) electrode placement (discussed within Subsection 5.6.1).

Notwithstanding later general discussion that naturally generates from figures 6e and 6f and associated results, since a detailed clinical study into stage 4 sleep is not an aim of the present study, these stage 4 sleep results are primarily intended to demonstrate repeatable linear characteristic outcomes of the new technique (that are in stark contrast to the chaotic-like first-order phase behaviour of conventional EEG frequency spectra. 

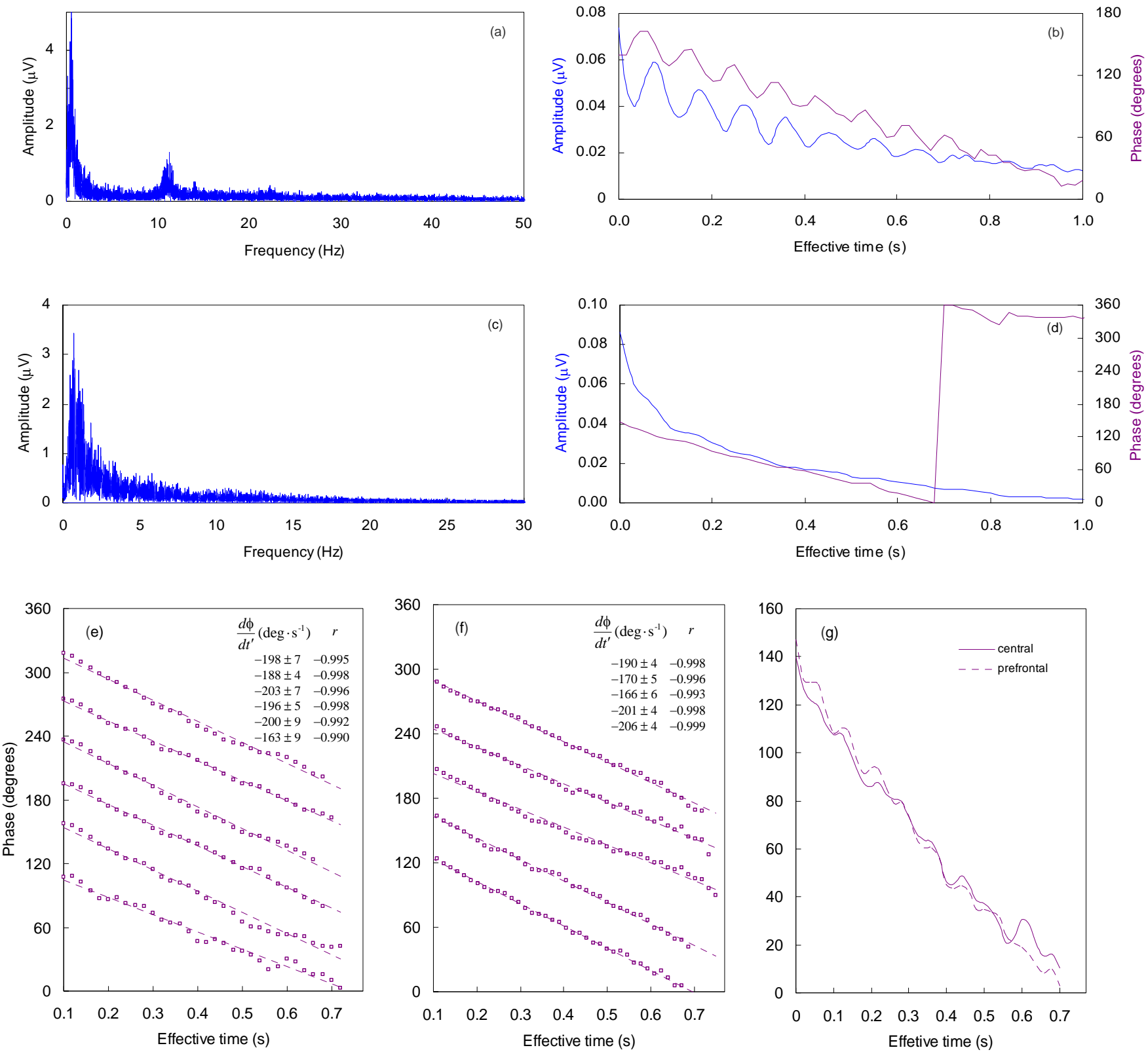

FIGURE 6. A conventional frequency spectrum (with alpha peak) for subject \#7 [58] categorised as awake/stage 1 sleep for occipital (O2) electrode placement is given in (a) with the corresponding double Fourier transformation of the EEG, or $F T^{\prime}$ spectrum, given in (b). A similar spectral pair for subject \#16 [58] in stage 4 sleep and central (C4) electrode placement are shown in (c) and (d). A linear regression of the phase profile in (d) over the domain $0.1 \leq t ' \leq 0.7 \mathrm{~s}$ is given in (e) (third-from-bottom trend line) which also stacks five other stage 4 sleep trend lines of the same EEG recoding (the six consecutive EEG segments with $10 \mathrm{~s}$ separation yield a total stage 4 sleep assessment time of 20.8 min and see main text for manner of incremental stacking via relative phase shifting), with regression values given in the figure legend. Similar stacked profiles for subject \#15 [59] in extended stage 4 sleep is given in (f). The first phase profile in (f) is zoomed within (g) along with a simultaneously collected phase profile for prefrontal (Fp2-F4) electrode placement. 
While not a focus of the present study ( $\Delta \phi$ analysis being the focus), the average (mean $\pm \mathrm{SD}$ ) oscillation frequency of five $F T^{\prime}$ amplitude profiles that contain notable oscillatory form (e.g., figures $5 b$ and $6 b$ ) is $9.7 \pm 1.8 \mathrm{~Hz}$.

Figures $7 \mathrm{a}$ and $7 \mathrm{~b}$ respectively show example conventional FFT and corresponding $F T^{\prime}$ spectra for an EEG segment of an awake subject in sleep preparedness and for occipital (O2) EEG; the relatively uniform FFT is without appreciable alpha or similar spectral peak. The smoothness of the $F T^{\prime}$ amplitude and phase profiles in figure 7, that reflect the FFT uniformity, is an atypical but nevertheless repeatable $F T^{\prime}$ characteristic. The decays of profile tails in figure 7 follow simple exponential functionality but overall are more accurately modelled via exponential functionality of the form $e^{-t^{\prime x} / \tau}$, where for a given profile $\tau$ is a standard time constant and the exponent, $x$, ranges from approximately 0.70 to 0.85 (again, $\Delta \phi$ analysis rather $F T^{\prime}$ profile decay functionality is the focus of the present study and therefore decay functionality is presented in a generalised context).
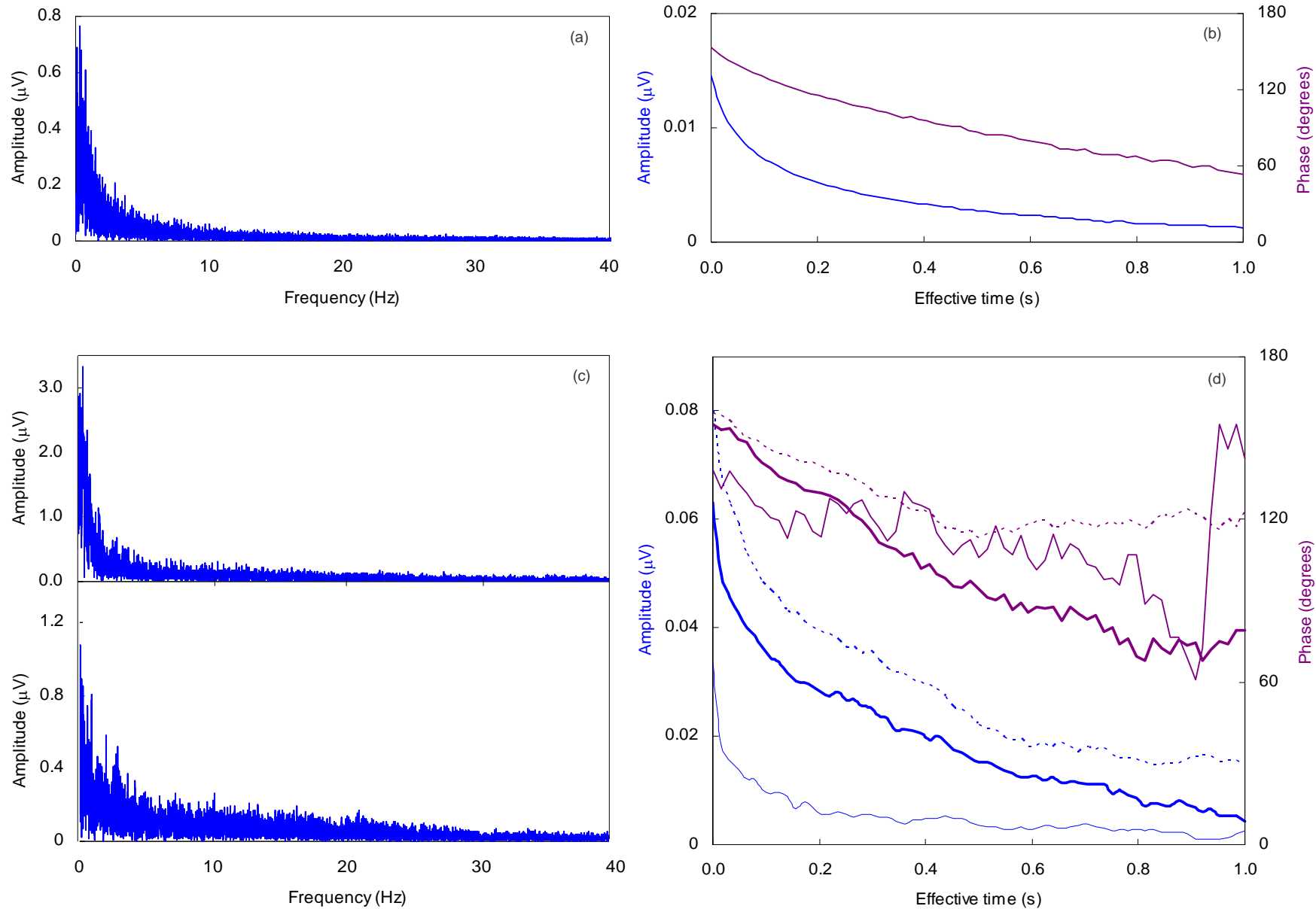

FIGURE 7. Conventional frequency spectra for subjects \#6 and \#9 [58] in sleep preparedness and for occipital (O2) electrode placement are respectively given in (a) and (c), with the two spectra of (c) associated with two separate EEG segments (recording times) for subject \#9. For each conventional frequency spectrum the corresponding (adjacent) double Fourier transformation of the EEG, or $F T^{\prime}$ spectrum, is respectively given in (b) and (d). Also shown by dashed lines in (d) are $F T^{\prime}$ profiles for central (C3) electrode placement for subject \#9, but again for a different EEG segment (corresponding FFT not shown but similar to those in (c)). 
Figure 7d shows three sets of similar profiles for another subject in sleep preparedness; one set associates with central (C3) electrode placement (dashed) and the other two sets associate with occipital (O2) electrode placement (all three sets have different EEG recording commencement times within the same polysomnographic record). Corresponding conventional FFT spectra are given in figure 7c for the occipital $F T^{\prime}$ profiles (the central FFT profile is not shown but is similar to the given occipital examples). While tabulations of select $\Delta \phi$ (ala example table A1) are not presented, the dominant discrete transition of $\Delta \phi=90^{\circ}$ in figure $7 \mathrm{~d}$, with slight symmetrical $\phi$ oscillation about the transitioned level, is worthy of note within the context of analysis and is called upon by later discussion. For broad comparison purposes only (a general rate trend for increasing sleep depth is later discussed), the $95 \%$ CI gradient for the phase profile of figure $7 \mathrm{~b}$ over a selected domain of interest $\left(0.2 \leq t^{\prime} \leq 1.0 \mathrm{~s}\right)$ is $d \phi / \mathrm{d} t^{\prime}=-74 \pm 2^{\circ} \cdot \mathrm{s}^{-1}(3 \%)$, based on an approximation of linear modelling $(r=-0.994, p<0.001)$.

\subsection{LEVEL $2 \Delta \phi$ DISTRIBUTION ANALYSES}

\subsubsection{CHI-SQUARED STATISTICAL ANALYSIS OF $\Delta \phi$ RADIALS}

Based on a frequency of occurrence distribution applied to all 87 upward $\Delta \phi$ radials of seven phase wheels collectively representing a cross-section of subjects and clinical states, including relatively short and long analysis times over which high-order phase relationships might occur, the most repeated $\Delta \phi$ values (rounded to integer values for a distribution resolution of $\delta \phi= \pm 0.5^{\circ}$ ) are given, together with their frequency of occurrence, in the two left-most columns of table 3. The two right-most columns present similar frequency of occurrence results but with $\Delta \phi$ values from 180 to $360^{\circ}$ (constituting 17 of the 87 radials) converted to a complementary angle from 0 to $180^{\circ}$ (i.e., $355^{\circ} \equiv 5^{\circ}$ and so on, with conversion rationale given within the Discussion).

The null hypothesis of an expected normal distribution of $\Delta \phi$ values is excluded via chi-squared analysis for which 36 out of 87 radials align with 16 out of a possible 360 polar angle values (two left-most columns), and 50 out of 87 radials align with 26 out of a possible 180 polar angle values (two right-most columns). Associated chi-squared values are respectively: $\chi^{2}=303, d f=12, p<0.001$ and $\chi^{2}=346, d f=7, p<0.001$.
Table 3. Frequency distributions of rounded $\Delta \phi$ values for radials from seven phase wheels combined.

\begin{tabular}{|c|c|c|c|}
\hline $\begin{array}{c}\Delta \phi \\
\text { (degrees) }\end{array}$ & $\begin{array}{c}\text { Number of } \\
\text { occurrences }\end{array}$ & $\begin{array}{c}\Delta \phi \\
\text { (degrees) }\end{array}$ & $\begin{array}{c}\text { Number of } \\
\text { occurrences }\end{array}$ \\
\hline $\begin{array}{c}\mathbf{5} \\
\mathbf{1 0} \& 11 \\
18 \\
\mathbf{2 0} \\
22 \\
27 \\
\mathbf{3 0} \\
33 \\
\mathbf{5 0} \\
59 \\
\mathbf{8 0} \& 81 \\
\mathbf{1 8 0}-182\end{array}$ & $\begin{array}{c}4 \\
4 \\
4 \\
3 \\
2 \\
3 \\
2 \\
5 \\
2 \\
2 \\
3 \\
3 \\
\text { 36 out of } 87 \\
\text { radials }\end{array}$ & $\begin{array}{c}4-6 \\
9-11 \\
18-23 \\
26-33 \\
50 \\
80-81 \\
178-180\end{array}$ & $\begin{array}{c}50 \text { out of } 87 \\
\text { radials }\end{array}$ \\
\hline
\end{tabular}

\subsubsection{BINOMIAL DISTRIBUTION STATISTICAL ANALYSIS OF $\triangle \phi$ RADIALS}

The following binomial distribution analysis considers primary radials at $5^{\circ}, 10^{\circ}, 20^{\circ}, 30^{\circ}, 45^{\circ}, 50^{\circ}, 60^{\circ}, 80^{\circ}, 90^{\circ}$, $120^{\circ}, 135^{\circ}, 150^{\circ}$ and $180^{\circ}$, with these primary radials based on landmark polar angles, individual phase wheel analysis, and the above frequency distribution results for all phase wheels combined. Note again that primary radials are provisionally defined and their ongoing refinement continues throughout this Subsection with finalisation rationale summarised within the Discussion.

For individual and combinations of phase wheels, the probability, $P$, of $\Delta \phi$ radials aligning with these primary radials is given within table 4 , for which $P$ is calculated in accordance with binomial probability theory and (1). Input parameters for (1), in addition to $m=13$ as set by the above-defined primary radials, are given in table 4 , and bracketed values within that table signify the effect of converting angles above $180^{\circ}$ to complementary angles in the range 0 to $180^{\circ}$. 


\begin{tabular}{|c|c|c|c|c|c|}
\hline Phase wheels & $\begin{array}{c}\text { Phase wheel clinical } \\
\text { descriptions }\end{array}$ & $\begin{array}{c}\text { Total number of } \\
\text { transitions, } H\end{array}$ & $\begin{array}{c}\text { Number of } \\
\text { alignments, } h \text {, } \\
\text { between transitions } \\
\text { and primary radials }\end{array}$ & $\begin{array}{l}\text { Alignment } \\
\text { resolution, } \pm \delta \phi \\
\quad \text { (degrees) }\end{array}$ & $\begin{array}{c}\text { Binomial probability } \\
\text { of observed } \\
\text { alignment, } P\end{array}$ \\
\hline 1 to 7 & All scenarios & 87 & 19 & 0.60 & $4.3 \times 10^{-9}$ \\
\hline 1 to 6 & $\begin{array}{l}\text { All scenarios excluding } \\
\text { approaching deep sleep }\end{array}$ & 80 & 19 & 0.60 & $9.8 \times 10^{-10}$ \\
\hline figure $4 \mathrm{e}$ & Healthy adult & 20 & 7 & 0.60 & $1.3 \times 10^{-5}$ \\
\hline figures $5 e+5 f$ & Probable Alzheimer's & 33 & 6 & 0.51 & 0.0010 \\
\hline 4,5 and 6 & Light and mid sleep & 28 & $6(8)$ & 0.50 & $3.7 \times 10^{-4}\left(9.7 \times 10^{-5}\right)$ \\
\hline 7 & Approaching deep sleep & 6 & 0 & 0.50 & 0.64 \\
\hline
\end{tabular}

\subsubsection{QUANTISED STRUCTURE ANALYSIS OF $\Delta \phi$ RADIALS}

Table 5 reveals identified Family $\Delta \phi_{c}$ values about which $\Delta \phi$ radials cluster and for which separations between clustering radials (Family members) can be written in terms of $k=j / 4$ or $j / 3(j=1,2,3 \ldots)$ multiples of a quantum increment value, $\alpha$. The $\alpha$ value determined for each Family, $\alpha_{\Delta \phi}$, is given within table 5 along with 95\% CI error margins, including for mean values, $\bar{\alpha}_{\Delta \phi}$. This Family $\Delta \phi_{c}$ and $\alpha_{\Delta \phi}$ analysis is based on the combined radials of seven phase wheels, and the previously described iterative linearization process of $\alpha_{\Delta \phi}$ determination which achieved correlation statistics of $r>0.99998, p<0.001$ for each Family.

Diagrams showing all radials and, where applicable, the separation between these radials in terms of $k \alpha_{\Delta \phi}$, are given for each Family in figure 8 (for which actual separation values align with their nominal $k \alpha_{\Delta \phi}$ form to within $1.0 \%$ unless the alignment is annotated as approximate). For these separations, table 6 gives the exact percentage difference between actual and nominal separations, with the average (mean $\pm \mathrm{SD}$ ) percentage difference for all Families combined being $0.39 \pm 0.28 \%$ (i.e., most considered alignments are well within the $1.0 \%$ acceptance margin).

Table 5. Quantum increment values, $\alpha_{\Delta \phi}$, for each Family of $\Delta \phi$ radials, with \pm error margins (EMs) at $95 \% \mathrm{Cl}$ including for mean values, $\bar{\alpha}_{\Delta \phi}$.

\begin{tabular}{|c|c|c|}
\hline $\begin{array}{c}\text { Family } \Delta \phi_{c} \\
\text { (degrees, radians) }\end{array}$ & $\begin{array}{c}\alpha_{\Delta \phi} \\
\text { (degrees) }\end{array}$ & $\begin{array}{c}\bar{\alpha}_{\Delta \phi} \text { EM } \\
\text { (degrees) }\end{array}$ \\
\hline $5, \pi / 36$ & $0.26534 \pm 0.00205$ & 0.00092 \\
$10, \pi / 18$ & $0.22734 \pm 0.00123$ & 0.00062 \\
$20, \pi / 9$ & $0.18193 \pm 0.00070$ & 0.00023 \\
$30, \pi / 6$ & $0.17685 \pm 0.00027$ & 0.00007 \\
$50,5 \pi / 9$ & $0.28509 \pm 0.00072$ & 0.00019 \\
$90, \pi / 2$ & $0.98495 \pm 0.00151$ & 0.00033 \\
$135,3 \pi / 4 *$ & $2.5329 \pm 0.0122$ & 0.0055 \\
$180, \pi$ & $4.9134 \pm 0.0259$ & 0.0116 \\
$220,11 \pi / 9 *$ & $7.7336 \pm 0.0604$ & 0.0302 \\
$270,3 \pi / 2$ & $11.981 \pm 0.090$ & 0.040 \\
\hline
\end{tabular}

* Considered non-primary or secondary Family.
Of the annotated radial separations in figure 8 that fall within the $1.0 \%$ acceptance margin, the percentage for each Family that involve only full- or half-integer $(k=j / 2)$ multiples of $\alpha_{\Delta \phi}$ are as follows in ascending (degree) Family order: 60, 100, 86, 71, 79, 91, 60, 71, 50 and 80\% (78\% for all Families combined). Similarly, the percentage for each Family that only involve full-integer multiples $(k=j)$ in ascending Family order are: 40, 75, 43, 36, 64, 19, 60, 29, 0.0 and 40\% (38\% for all Families combined). Hence, for the annotated radial separations a strong preference exists across all Families for full- or half-integer multiples of $\alpha_{\Delta \phi}$ over other fractional multiples of $\alpha_{\Delta \phi}\left(\chi^{2}=\right.$ $77, d f=1, p<0.001)$.

Note that within table 5 , the $\Delta \phi_{c}=135^{\circ}$ and $220^{\circ}$ Families are footnoted as non-primary or secondary Families (with remaining Families hence classified as primary). For the $135^{\circ}$ Family this relegation is based on the fact that all but one of the annotated separations involve a complementary radial (the only Family with an appreciable reliance on complementary radials). For the $220^{\circ}$ Family the relegation is based on relatively low occupancy and displaying the lowest percentage of full- or half-integer multiples of $\alpha$ within annotated radial separations (and furthermore being the only Family displaying no full-integer multiples). Also in contrast to other Families, these Families play lesser roles within table 7 which presents ratios of $\bar{\alpha}_{\Delta \phi}$, whereby ratio values align with nominal common fractions. The percentage differences between actual (exact median) and nominal ratio values are also presented within table 7 , along with actual ratio values with error margins at 95\% CI (following quadrature error combination). For that table, ratios are classified as: Tier 1 upon the formation of a simple common fraction involving primary Family members; Tier 2 upon the formation of a complex common fraction involving primary Family members; and Tier 3 upon the formation of a complex common fraction involving a secondary Family member. 
(a)

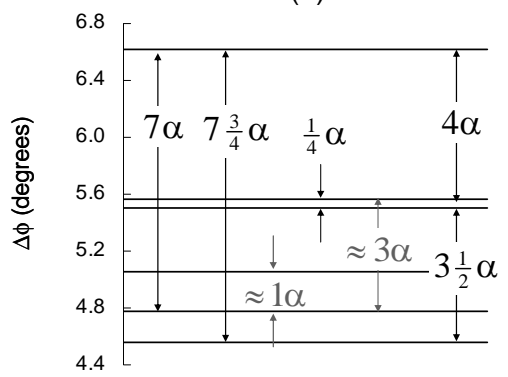

(b)

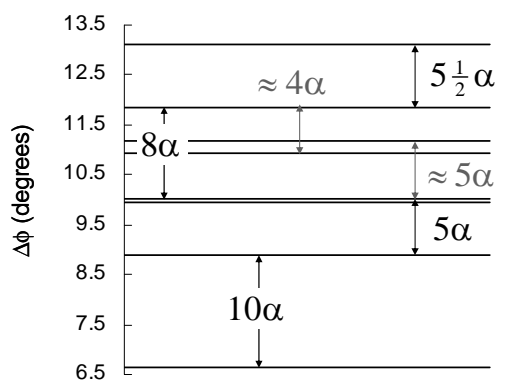

(g)

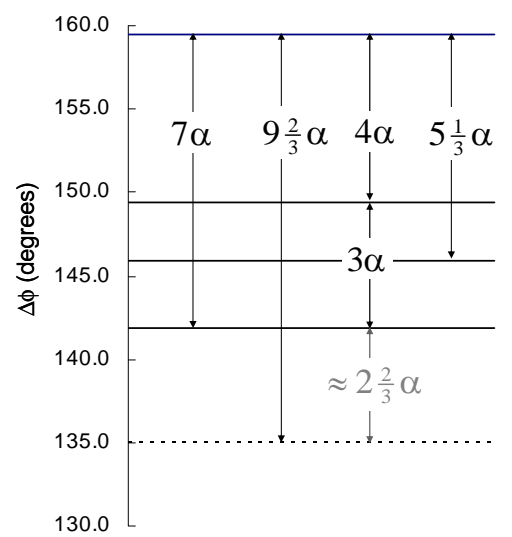

(h)

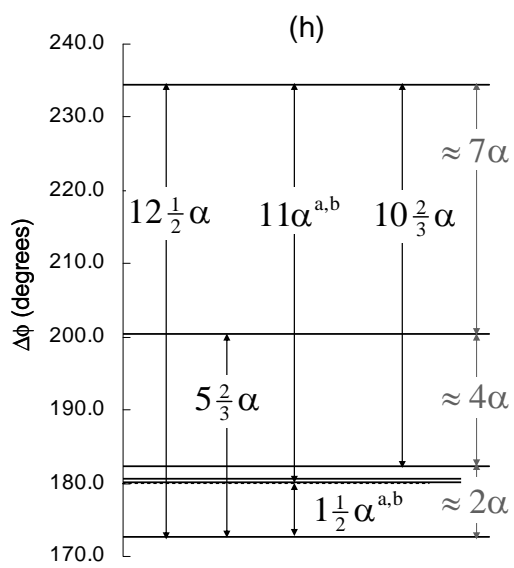

(c)

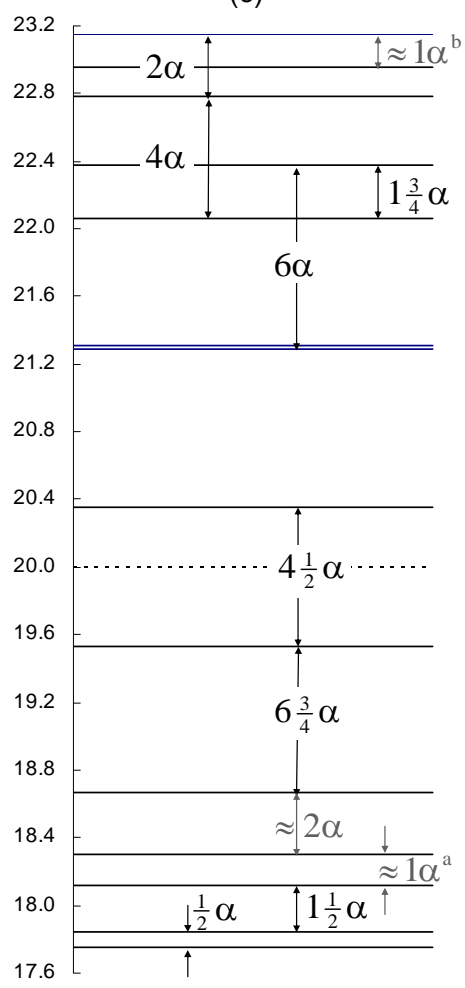

(d)

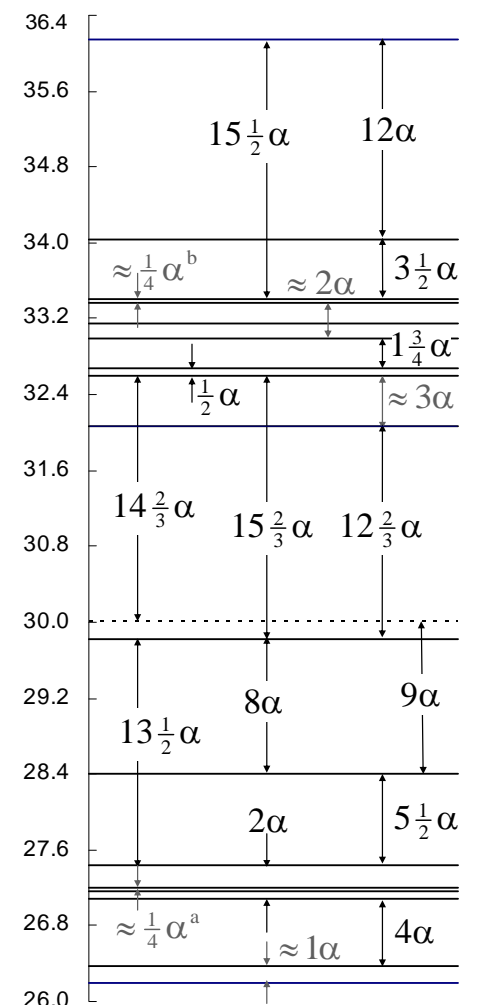

(e)

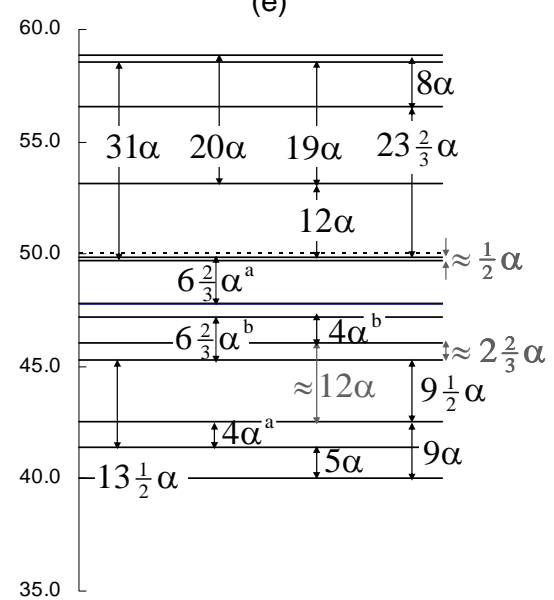

(f)

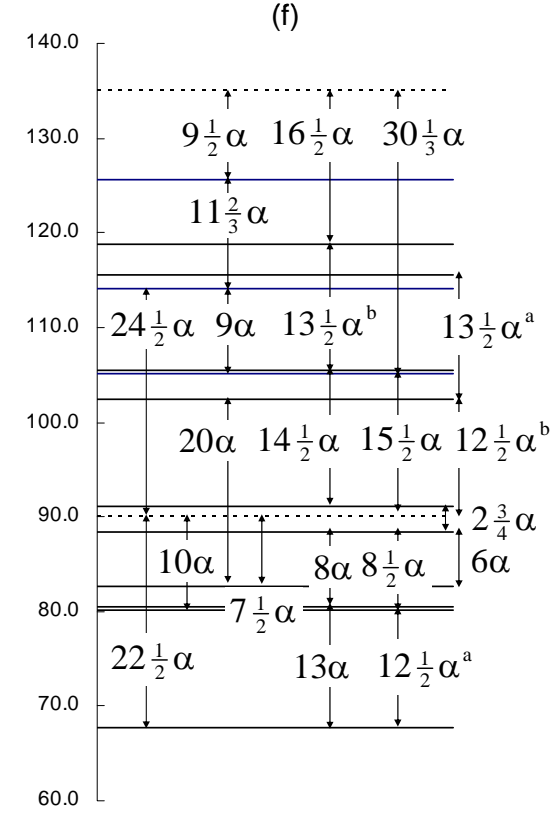

(i)

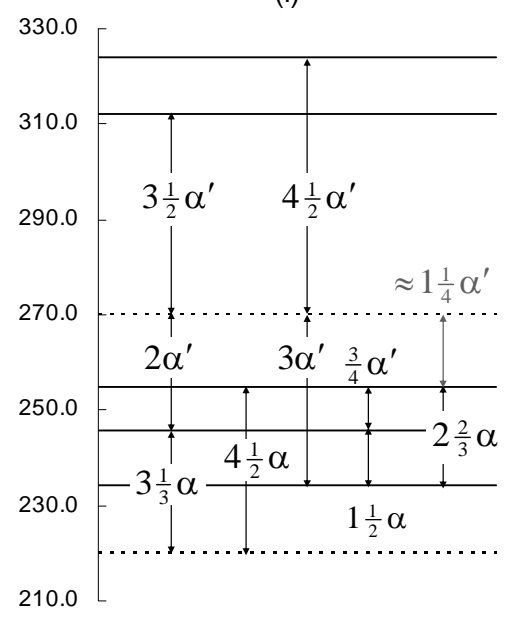

FIGURE 8. All $\Delta \phi$ radials from seven phase wheels (and thus seven $F T^{\prime}$ s) grouped within the Families of $\Delta \phi_{\mathrm{c}}=$ (a) $5^{\circ}$, (b) $10^{\circ}$, (c) $20^{\circ}$, (d) $30^{\circ}$, (e) $50^{\circ}$, (f) $90^{\circ}$, (g) $135^{\circ}$, (h) $180^{\circ}$, and (i) $220^{\circ} \& 270^{\circ}$ (combined). Shown for each Family are radial separations that align with integer and fractional multiples of some quantum transition value, $\alpha_{\Delta \phi}$ (see table 5 for $\alpha_{\Delta \phi}$ values). In (i) $\alpha$ and $\alpha^{\prime}$ respectively indicate $\alpha_{\Delta \phi}$ for the $220^{\circ}$ and $270^{\circ}$ Families. Each alignment is within $1.0 \%$ of its indicated nominal separation except if indicated as approximate. The percentage difference between actual and nominal separation values are given within table 6 . Navy transition radials signify complementary transitions as defined within the main text. 
Table 6. Data pertaining to the Family transition diagrams of figures 8a to 8i.

\begin{tabular}{|c|c|c|c|c|c|c|c|c|}
\hline $\begin{array}{c}\text { Family } \alpha \\
\text { Value } \\
\text { (degrees) }\end{array}$ & $\begin{array}{c}\text { Nominal } \\
\text { Radial } \\
\text { Separation }\end{array}$ & $\begin{array}{c}\text { \% Difference } \\
\text { From Actual } \\
\text { Separation }\end{array}$ & $\begin{array}{l}\text { Family } \alpha \\
\text { Value } \\
\text { (degrees) }\end{array}$ & $\begin{array}{c}\text { Nominal } \\
\text { Radial } \\
\text { Separation }\end{array}$ & $\begin{array}{c}\text { \% Difference } \\
\text { From Actual } \\
\text { Separation }\end{array}$ & $\begin{array}{c}\text { Family } \alpha \\
\text { Value } \\
\text { (degrees) }\end{array}$ & $\begin{array}{c}\text { Nominal } \\
\text { Radial } \\
\text { Separation }\end{array}$ & $\begin{array}{c}\text { \% Difference } \\
\text { From Actual } \\
\text { Separation }\end{array}$ \\
\hline \multicolumn{3}{|c|}{$\Delta \phi_{c}=5^{\circ}$ Family, figure 8a } & \multicolumn{3}{|c|}{$\Delta \phi_{c}=50^{\circ}$ Family, figure 8e } & \multicolumn{3}{|c|}{$\Delta \phi_{c}=135^{\circ}$ Family, figure $8 \mathrm{~g}$} \\
\hline \multirow[t]{7}{*}{0.26534} & & -0.13 & \multirow[t]{19}{*}{0.28509} & \multirow{8}{*}{$\begin{array}{c}19 \alpha \\
5 \alpha \\
23 \frac{2}{3} \alpha \\
9 \alpha \\
9 \frac{1}{2} \alpha \\
13 \frac{1}{2} \alpha \\
31 \alpha \\
4 \alpha^{a}\end{array}$} & \multirow{8}{*}{$\begin{array}{c}+0.024 \\
-0.17 \\
-0.17 \\
-0.19 \\
-0.20 \\
-0.20 \\
-0.20 \\
-0.21\end{array}$} & \multirow[t]{7}{*}{2.5329} & \multirow{7}{*}{$\begin{array}{c}9 \frac{2}{3} \alpha \\
3 \alpha \\
7 \alpha \\
4 \alpha \\
5 \frac{1}{3} \alpha \\
\approx 2 \frac{2}{3} \alpha\end{array}$} & +0.12 \\
\hline & $7 \frac{3}{4} \alpha$ & +0.37 & & & & & & -0.26 \\
\hline & $\frac{1}{4} \alpha$ & -0.51 & & & & & & -0.39 \\
\hline & $7 \alpha$ & -0.67 & & & & & & -0.48 \\
\hline & $3 \frac{1}{2} \alpha$ & +1.0 & & & & & & +0.63 \\
\hline & $\approx 3 \alpha$ & -1.4 & & & & & & +1.4 \\
\hline & $\approx 1 \alpha$ & +5.1 & & & & & & \\
\hline \multicolumn{3}{|c|}{$\Delta \phi_{c}=10^{\circ}$ Family, figure $8 \mathrm{~b}$} & & & & \multicolumn{3}{|c|}{$\Delta \phi_{c}=180^{\circ}$ Family, figure $8 \mathrm{~h}$} \\
\hline \multirow[t]{6}{*}{0.22734} & $5 \frac{1}{2} \alpha$ & +0.05 & & \multirow{11}{*}{$\begin{array}{c}8 \alpha \\
4 \alpha^{b} \\
12 \alpha \\
6 \frac{2}{3} \alpha^{a} \\
6 \frac{2}{3} \alpha^{b} \\
20 \alpha \\
\approx 2 \frac{2}{3} \alpha \\
\approx 12 \alpha \\
\approx \frac{1}{2} \alpha\end{array}$} & -0.21 & \multirow[t]{11}{*}{4.9134} & $5 \frac{2}{3} \alpha$ & -0.15 \\
\hline & $8 \alpha$ & -0.15 & & & +0.23 & & $12 \frac{1}{2} \alpha$ & +0.43 \\
\hline & $10 \alpha$ & +0.25 & & & -0.56 & & $11 \alpha^{a}$ & -0.53 \\
\hline & $5 \alpha$ & -0.68 & & & +0.60 & & $11 \alpha^{b}$ & +0.60 \\
\hline & $\approx 5 \alpha$ & +2.0 & & & +0.65 & & $10 \frac{2}{3} \alpha$ & -0.62 \\
\hline & & +2.5 & & & +0.97 & & $1 \frac{1}{2} \alpha^{a}$ & +0.73 \\
\hline \multicolumn{3}{|c|}{$\Delta \phi_{c}=20^{\circ}$ Family, figure 8c } & & & +1.2 & & $1 \frac{1}{2} \alpha^{b}$ & -0.76 \\
\hline \multirow[t]{11}{*}{0.18193} & $\frac{1}{2} \alpha$ & +0.038 & & & +1.5 & & $I_{2}$ & +1.1 \\
\hline & $6 \frac{3}{4} \alpha$ & +0.16 & & & +3.1 & & $\begin{array}{l}\approx 4 \alpha \\
\approx 7 \alpha\end{array}$ & -1.5 \\
\hline & $1 \frac{3}{4} \alpha$ & +0.20 & & & & & & -2.3 \\
\hline & $1 \frac{1}{1} \alpha$ & -0.33 & & & & & & \\
\hline & $\perp_{2} \alpha$ & -0.33 & \multicolumn{3}{|c|}{$\Delta \phi_{c}=90^{\circ}$ Family, figure 8f } & \multicolumn{3}{|c|}{$\Delta \phi_{c}=220^{\circ}$ Family, figure $8 \mathrm{i}$} \\
\hline & $\begin{array}{l}6 \alpha \\
4 \alpha\end{array}$ & -0.51 & \multirow[t]{26}{*}{0.98495} & \multirow{7}{*}{$\begin{array}{c}2 \frac{3}{4} \alpha \\
7 \frac{1}{2} \alpha \\
12 \frac{1}{2} \alpha^{a} \\
14 \frac{1}{2} \alpha \\
24 \frac{1}{2} \alpha \\
13 \frac{1}{2} \alpha^{a}\end{array}$} & \multirow{7}{*}{$\begin{array}{r}-0.023 \\
+0.025 \\
-0.080 \\
-0.082 \\
-0.10 \\
-0.11\end{array}$} & 7.7336 & $4 \frac{1}{2} \alpha$ & +0.10 \\
\hline & $4 \frac{1}{2} \alpha$ & +0.77 & & & & & $3 \frac{1}{3} \alpha$ & +0.43 \\
\hline & $2 \alpha$ & -0.79 & & & & & $1 \frac{1}{2} \alpha$ & -0.68 \\
\hline & $\approx 2 \alpha$ & +1.7 & & & & & $2 \frac{2}{2} \alpha$ & -0.75 \\
\hline & $\approx 1 \alpha^{a}$ & -2.2 & & & & & & \\
\hline & $\approx 1 \alpha^{b}$ & -2.7 & & & & & & \\
\hline$\Delta \phi_{c}$ & $30^{\circ}$ Family, & ure 8d & & & & $\Delta \phi_{c}$ & $270^{\circ}$ Family, & ig. 8(i) \\
\hline 0.17685 & $12 \frac{2}{3} \alpha$ & +0.040 & & $8 \frac{1}{2} \alpha$ & +0.12 & 11.981 & $4 \frac{1}{2} \alpha$ & -0.11 \\
\hline & $15 \frac{1}{2} \alpha$ & -0.043 & & $9 \frac{1}{2} \alpha$ & +0.13 & & $\frac{3}{4} \alpha$ & -0.43 \\
\hline & $14^{2} \alpha$ & -0.069 & & 2 & -0.13 & & $2 \alpha$ & +0.62 \\
\hline & $2 \alpha$ & +0.085 & & $30 \frac{1}{2} \alpha$ & -0.17 & & $3 \frac{1}{2} \alpha$ & +0.63 \\
\hline & $12 \alpha$ & -0.10 & & $16 \frac{1}{2} \alpha$ & +0.19 & & $3 \alpha$ & -0.87 \\
\hline & $9 \alpha$ & +0.15 & & $20 \alpha$ & +0.27 & & $\approx 1 \frac{1}{4} \alpha$ & +1.2 \\
\hline & $1 \frac{3}{4} \alpha$ & +0.17 & & $22 \frac{1}{2} \alpha$ & +0.29 & & & \\
\hline & $13 \frac{1}{2} \alpha$ & +0.23 & & $11_{4}^{3} \alpha$ & +0.29 & & & \\
\hline & $15^{\frac{2}{3}} \alpha$ & -0.31 & & $12 \frac{1}{2} \alpha^{b}$ & +0.30 & & & \\
\hline & $5 \frac{1}{2} \alpha$ & -0.38 & & $13 \frac{1}{2} \alpha^{b}$ & -0.57 & & & \\
\hline & $8 \alpha$ & +0.65 & & $13 \alpha$ & -0.68 & & & \\
\hline & $\frac{1}{2} \alpha$ & +0.65 & & $15 \frac{1}{2} \alpha$ & +0.72 & & & \\
\hline & $3 \frac{1}{2} \alpha$ & +0.81 & & $10 \alpha$ & +0.90 & & & \\
\hline & $4 \alpha$ & +0.93 & & $9 \alpha$ & +0.93 & & & \\
\hline & $\approx \frac{1}{4} \alpha^{a}$ & +1.8 & & $\begin{array}{l}8 \alpha \\
6 \alpha\end{array}$ & -0.99 & & & \\
\hline & $\approx \frac{1}{4} \alpha^{b}$ & +1.8 & & & & & & \\
\hline & $\approx 3 \alpha$ & -1.8 & & & & & & \\
\hline & $\approx 1 \alpha$ & -3.9 & & & & & & \\
\hline & $\approx 2 \alpha$ & +4.6 & & & & & & \\
\hline
\end{tabular}


Table 7. Ratios of $\bar{\alpha}_{\Delta \phi}$ values and their alignment with common fractions, categorised within three Tiers based on common fraction type (simple or complex) and Family type (primary or secondary). Tier 1 involves simple common fractions and primary Family members only. Here, a simple fraction is defined as being constructible solely from natural numbers less than 10 .

\begin{tabular}{|c|c|c|c|}
\hline \multicolumn{4}{|c|}{ Tier 1 Ratio Alignment\# } \\
\hline Family $\alpha$ Ratio & Nominal Ratio Value & $\begin{array}{c}\text { \% Difference Magnitude } \\
\text { From Actual (Median) } \\
\text { Ratio Value }\end{array}$ & $\begin{array}{c}\text { Actual Ratio Value } \\
\pm 95 \% \text { CI }\end{array}$ \\
\hline $\bar{\alpha}_{5^{0}}: \bar{\alpha}_{30^{0}}$ & $3: 2$ & 0.024 & $0.6665 \pm 0.0023$ \\
\hline $\bar{\alpha}_{10^{0}}: \bar{\alpha}_{20^{0}}$ & $5: 4$ & 0.032 & $0.8003 \pm 0.0024$ \\
\hline $\bar{\alpha}_{10^{0}}: \bar{\alpha}_{50^{0}}$ & $4: 5$ & 0.32 & $1.2540 \pm 0.0035$ \\
\hline $\bar{\alpha}_{90^{0}}: \bar{\alpha}_{180^{0}}$ & $1: 5$ & 0.23 & $4.988 \pm 0.0119$ \\
\hline $\bar{\alpha}_{10^{0}}: \bar{\alpha}_{30^{0}}$ & $9: 7$ & 0.018 & $0.7779 \pm 0.0021$ \\
\hline \multicolumn{4}{|c|}{ Tier 2 Ratio Alignment } \\
\hline $\bar{\alpha}_{20^{0}}: \bar{\alpha}_{180^{0}}$ & $1: 27$ & 0.026 & $27.01 \pm 0.07$ \\
\hline $\bar{\alpha}_{50^{0}}: \bar{\alpha}_{270^{0}}$ & $1: 42$ & 0.060 & $42.03 \pm 0.14$ \\
\hline $\bar{\alpha}_{5^{0}}: \bar{\alpha}_{270^{0}}$ & $1: 45$ & 0.34 & $45.15 \pm 0.22$ \\
\hline $\bar{\alpha}_{5^{0}}: \bar{\alpha}_{180^{0}}$ & $1: 181 / 2$ & 0.093 & $18.52 \pm 0.08$ \\
\hline $\bar{\alpha}_{20^{0}}: \bar{\alpha}_{30^{0}}$ & $181 / 2: 18$ or $18: 171 / 2$ & 0.092 or 0.015 & $0.9721 \pm 0.0013$ \\
\hline $\bar{\alpha}_{5^{0}}: \bar{\alpha}_{10^{0}}$ & $21: 18$ & 0.042 & $0.8568 \pm 0.0038$ \\
\hline $\bar{\alpha}_{30^{0}}: \bar{\alpha}_{50^{0}}$ & $18: 29$ & 0.058 & $1.612 \pm 0.001$ \\
\hline $\bar{\alpha}_{5^{0}}: \bar{\alpha}_{50^{0}}$ & $27: 29$ or $21: 22 \frac{1}{2}$ & 0.033 or 0.017 & $1.074 \pm 0.004$ \\
\hline $\bar{\alpha}_{20^{0}}: \bar{\alpha}_{50^{0}}$ & $18 \frac{1}{2}: 29$ or $16: 25$ & 0.034 or $0.29 *$ & $1.567 \pm 0.002$ \\
\hline \multicolumn{4}{|c|}{ Tier 3 Ratio Alignment } \\
\hline $\bar{\alpha}_{5^{0}}: \bar{\alpha}_{135^{0}}$ & $2: 18$ & 0.48 & $9.546 \pm 0.039$ \\
\hline $\bar{\alpha}_{20^{0}}: \bar{\alpha}_{135^{0}}$ & $1: 14$ & 0.55 & $13.92 \pm 0.03$ \\
\hline $\bar{\alpha}_{10^{0}}: \bar{\alpha}_{220^{0}}$ & $1: 34$ & 0.052 & $34.02 \pm 0.16$ \\
\hline $\bar{\alpha}_{220^{0}}: \bar{\alpha}_{270^{0}}$ & $20: 31$ & 0.051 & $1.549 \pm 0.008$ \\
\hline
\end{tabular}

For the Tier $1 \bar{\alpha}_{\Delta \phi}$ ratios of table 7 , the percentage differences between actual and nominal ratio values, for CIs ranging from 80 to $95 \%$, are graphically indicated by figure B1 (Appendix B). Figure B1 demonstrates that for all Tier 1 ratio calculations, percentage differences remain within $0.5 \%$ when $95 \%$ CI error margins (and not just exact median $\bar{\alpha}_{\Delta \phi}$ values) are included.

Figure 9 plots the $\bar{\alpha}_{\Delta \phi}$ versus $\Delta \phi_{c}$ values of table 5 , with vertical error bars at 95\% CI just resolvable (and therefore included) for the two right-most points only. The parabolic curve of best fit for figure 9 ( $r=0.99998, p<0.001)$, for which error margins are stated at $95 \% \mathrm{CI}$, is given by (2):

$$
\begin{aligned}
\bar{\alpha}_{\Delta \phi} & =(1.9772 \pm 0.0049) \times 10^{-4} \Delta \phi_{c}^{2} \\
& -(9.990 \pm 0.097) \times 10^{-3} \Delta \phi_{c}+(3.002 \pm 0.021) \times 10^{-1},
\end{aligned}
$$

with a subsequent rate of change of:

$$
\begin{aligned}
\frac{d \bar{\alpha}_{\Delta \phi}}{d \Delta \phi_{c}} & =(3.954 \pm 0.010) \times 10^{-4} \Delta \phi_{c} \\
& -(9.990 \pm 0.097) \times 10^{-3} .
\end{aligned}
$$

Note that the uncertainty analysis applied to the coefficients of the above inter-Family parabolic modelling includes the propagation of $\bar{\alpha}_{\Delta \phi} 95 \%$ CI error margins, but not standard error based on the variance of the model's $y$ - 
residuals. The rationale for this approach is found within the Evidence of a Quantum Nature Subsection 5.5. In any event, the standard errors of the coefficients from left to right (second- to zeroth-order function terms) based on $y$ variance analysis are small due to the model's strong correlation (standard errors being $\pm 1.5 \times 10^{-6}$ degrees $^{-1}$, $\pm 4.0 \times 10^{-4}$ and $\pm 1.8 \times 10^{-2}$ degrees respectively) .

The $x$-turning point of the figure 9 parabola minimum is $\Delta \phi_{\min }=25.33 \pm 0.25^{\circ}$ and the corresponding $y$-turning point is $\alpha_{\min } \approx 0.1740^{\circ}$. Here, $\alpha_{\min }$ is indicated as approximate based on an outcome-driven postulate that can now be formulated. Viz., just like many intra-Family radial separations are multiples of $k \alpha_{\Delta \phi}$ as per figure 8 and table 6, it may be assumed that $\alpha_{\min }$ has significance and that some so-far-unaccounted-for separations are multiples of $k \alpha_{\min }$, in which case $\alpha_{\min }$ can be determined with high accuracy using the previously applied iterative linearization process.

For this process applied about the region of approximated $\alpha_{\min }$ and with the separation acceptance/rejection threshold set finer via its reduction from $1.0 \%$ (used for $\alpha_{\Delta \phi}$ determination) to $0.33 \%$ analysis gives $\alpha_{\min }=0.174019 \pm 0.000054^{\circ}$ with an $\bar{\alpha}_{\Delta \phi} \quad$ EM of $\pm 0.000010^{\circ} \quad(95 \% \quad \mathrm{CI}$ and correlation statistics of $r>0.99999, \quad p<0.001)$, based on a total of 28 $k \alpha_{\min }$ matches across five Families (especially within the $20^{\circ}, 30^{\circ}$ and $50^{\circ}$ Families). The average percentage difference (magnitude) between actual and nominal $\left(k \alpha_{\min }\right)$ separation values for the 28 matches is less than $0.2 \%$. See figure C1 (Appendix C) for transition diagram (with linearization graph of $\alpha_{\min }$ determination as inset). Due to the wide-scoping manner in which $\alpha_{\min }$ spans across Families, it will from this point be referred to as a universal quantum increment value.

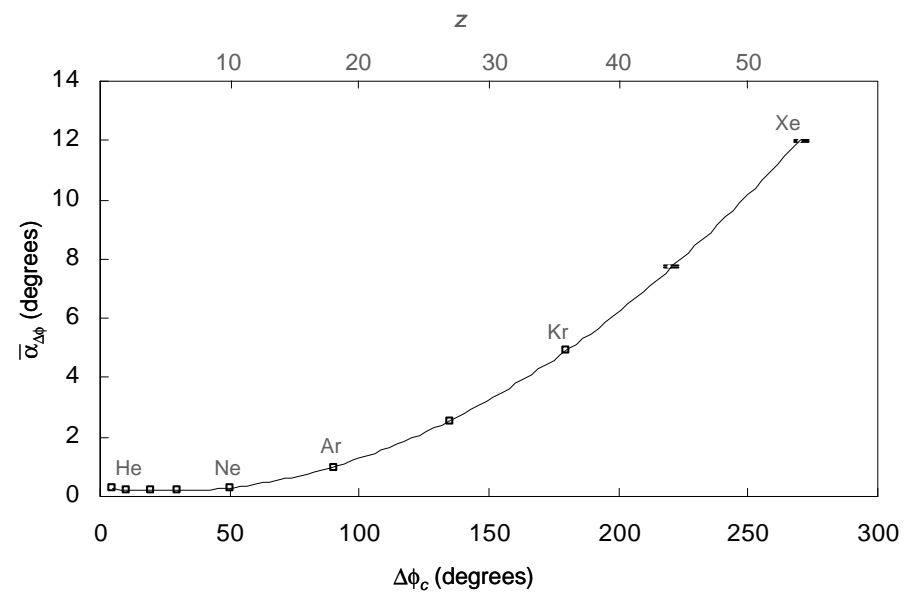

FIGURE 9. Family $\bar{\alpha}_{\Delta \phi}$ versus $\Delta \phi_{c}$ based on the values of table 5. The secondary $x$-axis applies the linear transformation $\Delta \phi_{c} / 5 \rightarrow z$, with subsequent annotations identifying $z$ values for (five of eight) primary Family members that coincide with the atomic numbers of indicated noble (rare) gases. Error bars $(95 \% \mathrm{Cl})$ are resolvable (just) for the two right-most points only.
Finally, the remaining tentative result below is not intended for in-depth analysis but as a brief point of interest and potential relevance within later quantum mechanical discussions:

Linear mapping to a proposed dimensionless index, $z$, via the transformation $\Delta \phi_{c} / 5 \rightarrow \mathrm{z}$ (as per the secondary $x$-axis of figure 9), respectively yields for the eight ascending primary Family members $z=1,2^{*}, 4,6,10^{*}, 18^{*}, 36^{*}$ and $54^{*}$. The $z$ values with asterisk equal the number of electrons for the most stable electronic configurations of atomic structure theory such that all subshells below any given s-subshell are fully occupied. That is, these asterisked $z$ values equate to the atomic numbers of the noble (rare) gas elements of $\mathrm{He}\left(1 \mathrm{~s}^{2}\right), \mathrm{Ne}\left(2 \mathrm{p}^{6}\right), \operatorname{Ar}\left(3 \mathrm{p}^{6}\right)$, $\mathrm{Kr}\left(4 p^{6}\right)$, and $\mathrm{Xe}\left(5 p^{6}\right)$, as annotated within figure 9 , where the bracketed notation gives the filling of the outer-most subshell. Following the same atomic labelling, the remaining primary Family $z=1,4,6$ values equate to the elements of $\mathrm{H}\left(1 \mathrm{~s}^{1}\right), \operatorname{Be}\left(2 \mathrm{~s}^{2}\right)$, and $\mathrm{C}\left(2 \mathrm{p}^{2}\right)$, whilst the turning point equates to $z=5.07 \pm 0.05$ or $\approx B\left(2 p^{1}\right)$.

\section{DISCUSSION}

\subsection{ELIMINATION OF ARTEFACT AS A CAUSAL EFFECT}

Given the known chaotic-like nature of harmonic phase for conventional EEG frequency analysis (first-order analysis), and chaotic elements and limitations of existing phasebased analysis techniques such as second-order bispectral analysis discussed within Subsection 1.2, the identification of any strong, or even somewhat regular, phase relationship or behaviour within a new manner of EEG analysis prompts inherent interest and calls for closer inspection. However, mathematically, spectral oscillations in response to some sharp peak are expected, and so caution must be followed when assigning any biophysical significance to $F T^{\prime}$ profiles that exhibit oscillations (e.g., figures $4 \mathrm{~b}$ and $6 \mathrm{~b}$ ) originating from a conventional FFT peak of high alpha (or other such) activity. Hence, consideration must first be given to the possibility of $F T^{\prime}$ spectral characteristics being some not surprising mathematical result or artifact, despite various layers of statistical analysis demonstrating that $\Delta \phi$ behaviours within presented $F T^{\prime}$ spectra defy normal distribution expectations. These possibilities are eliminated as follows:

Firstly, as per Subsection 1.3, the calcium signalling study [55] demonstrates that $\mathrm{Ca}^{2+}$ ions regulate a wide spectrum of cellular processes, and that $\mathrm{Ca}^{2+}$ concentration oscillatory patterns display large spatial and temporal diversity originating from an intricate concert action between several $\mathrm{Ca}^{2+}$ transporters to provide important cellular communication encoding. Also, various forms of coupling, including oscillatory forms, across frequencies of the EEG have been identified as per Subsections 1.2 and 1.3. Hence, suggestions that the oscillatory nature of 
presented $F T^{\prime}$ spectra, and the oscillatory FFT deconstruction of figure 3, have some biophysical connectedness to impulse encoding is reasonable through analogous consistency, especially given the ion transport origin of the EEG in the case of the $\mathrm{Ca}^{2+}$ analogy.

Secondly, while the decaying behaviour of $F T^{\prime}$ amplitude profiles are reminiscent of a FFT spectral leakage response to windowing [64], such spectral leakage is typically smaller in scale ( -60 to $-80 \mathrm{~dB}$ compared to -10 to $-20 \mathrm{~dB}$ for the present study). Also, the main $F T^{\prime}$ amplitude profile characteristics display relative invariance to window testing and are not apparent for random waveform (e.g., white noise) analysis, with the long duration of analysed EEG segments (up to $200 \mathrm{~s}$ ) also lessening the significance of windowing. Hence, windowing effect mitigation, together with the consistency of several $F T^{\prime}$ features for different subjects, EEG states and FFT defining parameters (such as $\Delta f=0.125$ versus $0.005 \mathrm{~Hz}$ and $T=8$ versus $200 \mathrm{~s}$ ), further suggest a biophysical basis of $F T^{\prime}$ characteristics.

Thirdly, aliasing artifact due to a Nyquist-related effect of FFT sampling may be eliminated since $F T^{\prime}$ amplitude profiles have mostly decayed once an effective time of $t^{\prime} \approx 1.0 \mathrm{~s}$ is reached. Therefore, the Nyquist sampling theorem of $f_{s} \geq 2 f_{\max }$, which when written in a time base for FFT sampling dictates a $T \geq 2.0 \mathrm{~s}$ condition for the stated effective decay time, is easily met by both data sets.

Finally, within the above-stated mathematical expectation of spectral oscillations in response to some sharp peak lies an important point of perspective. Viz., within clinical neurophysiology, when an alpha or other peak is observed it may be associated with some state of consciousness, increased neural activity and/or memory recall, perhaps with quantitative considerations such as contained relative power and the physical characteristics of a causal sinusoidal oscillation in the original time-domain. More mathematically-orientated considerations (e.g., via EEG simulation models) naturally provide additional quantified explanation of these peaks (e.g., a relatively early explanation, that still holds some relevancy, involves a cortical system modelled as a closed medium allowing the propagation of travelling waves and thus capable of standing waves and resonance [4]). However, despite the many valuable clinical and mathematical neurophysiological insights provided to date, including from quite sophisticated EEG simulation models [4,5], biophysical origin specificity for such peaks still arguably requires completion of explanation.

In contrast, within a highly mathematical field such as quantum mechanics (as a deliberate comparative example due to later discussion), for any frequency spectrum that contains a sharp resonance peak, the peak is invariably traced to a specific and well mathematically understood physical origin. Thus, rather than taking a perspective that an alpha or other conventional FFT peak will naturally bring about spectral oscillation within the $F T^{\prime}$, a reverse perspective of asking what underlying functional, biophysical behaviour within the FT' is giving rise to the FFT peak?, is worthy of exploration. Certainly, the apparent deliberate $\phi$ structuring and functionality over the effective $1 \mathrm{~s}$ timescale of $F T^{\prime}$ amplitude profiles implies biophysical meaningfulness, especially since the $1 \mathrm{~s}$ timescale (which may still reflect real-time behaviour) is consistent with the period of average human heart rate and so may broadly reflect expected body homeostasis [33].

\subsection{INCREASING LINEARITY OF $\phi$ AS A FUNCTION OF SLEEP DEPTH}

For awake and non-deep sleep states, upward $\Delta \phi$ values of any given $F T^{\prime}$ spectrum are spread by varying amounts from 0 to $2 \pi$ radians (most spread for awake), with such spectra also displaying oscillatory $\phi$ behaviour in the presence of alpha and other spectral peaks within the conventional FFT (recall that a target comparison state of the present study is occipital EEG with eyes-closed, due to the state's known association with strong PDR alpha activity and alpha linkage to neural network communication and co-ordination [4,51,61], as per Subsection 3.3.2).

Reductions in both the spread of $\Delta \phi$ values, and the oscillatory nature of $\phi$, are observed for increasing relaxation and sleep depth, and associate with a progressive increase in $\phi$ versus $t^{\prime}$ linearity. While these increasing linearity observations are generalised and with exceptions (due to the influence on the $F T^{\prime}$ spectrum by spectral peaks within, and overall smoothness of, the conventional FFT), the high degree of linearity for stage 4 sleep is more absolute (note that despite this commentary it is actually the $F T^{\prime}$ spectrum that evidentially influences the FFT spectrum as suggested within Subsection 5.1).

The rate magnitude $\left|d \phi / \mathrm{d} t^{\prime}\right|$ similarly appears to generally increase with progression towards stage 4 sleep. For example, the $F T^{\prime}$ spectrum of figure $4 \mathrm{~d}$ for a subject in relaxed wakefulness with eyes-closed, derived from a prefrontal EEG (and so physiologically linked to attention and short-term memory), while displaying some degree of $\phi$ irregularity, demonstrates approximate $\phi$ constancy or low $\left|d \phi / \mathrm{d} t^{\prime}\right|$. The behavioural characteristic of figure $4 \mathrm{~d}$ is repeated to varying degrees by figures $7 b$ and $7 d$ for subjects in sleep preparedness. The generalised picture of increasing $\left|d \phi / \mathrm{d} t^{\prime}\right|$ with increasing sleep depth continues with figure $6 \mathrm{~b}$ for awake/stage 1 sleep, and is completed by the more in depth stage 4 sleep (linearity) analysis based on three subjects and 17 EEG segments. For this latter analysis $(-r \geq 0.990, p<0.001$ in all cases $), d \phi / \mathrm{d} t^{\prime}$ values for the three subjects are: $-163 \pm 9$ to $-203 \pm 7^{\circ} \cdot \mathrm{s}^{-1}(3.3 \%)$, $-166 \pm 6$ to $-206 \pm 4^{\circ} \cdot \mathrm{s}^{-1} \quad(3.7 \%)$, and $-190 \pm 5$ to $-236 \pm 8^{\circ} \cdot \mathrm{s}^{-1}(2.5 \%)$, with bracketed values giving the average of all intra-figure 95\% CI error margins when the margins are expressed as a percentage (see figures 6e and $6 f)$. Stage 2 and 3 sleep $F T^{\prime}$ spectra that are not shown also complement the described overall characteristic behaviour. 
The ranging $d \phi / \mathrm{d} t^{\prime}$ values for deep sleep are suggestive of dynamically changing, structured complexity within outwardly appearing constancy (perfect constancy of linearity would after all be an over-simplified expectation if some linear outcome was hindsighted). It is not uncommon for the tail of an EEG's conventional amplitude versus frequency spectrum to possess some degree of linearity depending on a subject's clinical state. Nevertheless (and despite the slight confounding effect of superimposed oscillations in some cases), the quantified linearities above are remarkable given the chaotic-like behaviour of harmonic phase for conventional first-order FFT spectra, and the limitations of established phase-based analysis techniques such as bispectral analysis. The remarkableness increases upon contemplation of physical significance, since the outcome indicates that the phases of successive harmonics of the figure 3 deconstruction are linearly related across the frequency spectrum, thus demonstrating further complexity within the outwardly simple linear form.

\subsection{OSCILLATORY FT'BEHAVIOUR}

The oscillatory nature of $F T^{\prime}$ amplitude and phase profiles (that clearly follow one another), especially observed in the presence of alpha and other spectral peaks of EEG activity, draws parallel with a PSK study [2] demonstrating the data communication benefits of controlled phase oscillation in the time-domain, with such control for just one conventional harmonic leading to the provision of discretely encoded data transmission rates of up to $8 \mathrm{Mbit}^{-1} \mathrm{~s}^{-1}$ (and higher for approach variants). The manner of $\phi$ oscillation across the $t^{\prime}$ domain within the present study is comparatively complex and demonstrates several degrees-of-freedom that seemingly include deliberately structured upward, downward and horizontal $\Delta \phi$ (upward remaining the primary focus), as well as oscillation frequency variability with the oscillation frequencies of a selection of $F T^{\prime}$ amplitude profiles being $9.7 \pm 1.8 \mathrm{~Hz}$. Recalling that fluctuations in the original time-domain manifest within the $F T^{\prime}$ spectrum, albeit in potentially complex ways, these reported oscillation frequencies are consistent with those of alpha and spindle peaks and so give further credence to the Subsection 5.1 position that $F T^{\prime}$ characteristics are physically meaningful. Following the parallel comparison with [2], here such high degrees-offreedom within the manner of $\phi$ oscillation control would lead to discretely encoded data transmission rates of immense potential consistent with that expected of an extraordinarily sophisticated communications platform like the CNS.

Thus, the showcased $\phi$ oscillations, by their evidential deliberate nature and ion transport origin are indeed broadly consistent with the previously raised calcium signaling whereby $\mathrm{Ca}^{2+}$ concentration oscillatory patterns provide important cellular communication encoding [55]. Together with the physical interpretation provided by figure 3 , the structure of these $\phi$ oscillations further elucidate the physical and mathematical nature of high-order, acrossfrequency EEG relationships that manifest as subtle periodic contours of high second-order phase coupling across bicoherence spectra as outlined within Subsections 1.2 and 1.3. This elucidation includes revelation of why a conventional EEG frequency spectrum typically appears so variable, for both intra- and inter-subject comparisons of the same neural state, since what was considered chaoticlike amplitude variability for any given harmonic of the conventional frequency spectrum, is now revealed as a harmonic amplitude constructed from multi-ordered states of oscillation (or harmonics across the frequency spectrum) as per figure 3 .

\subsection{PHASE WHEEL SECTOR RATIOS AND RADIAL DISTRIBUTIONS}

The $\Delta \phi$ values that become the phase wheel radials belong to Families named for the polar angles about which the radials cluster. Primary Families are identified at $\Delta \phi_{c}=5^{\circ}$, $10^{\circ}, 20^{\circ}, 30^{\circ}, 50^{\circ}, 90^{\circ}, 180^{\circ}$ and $270^{\circ}$, and secondary Families at $\Delta \phi_{c}=135^{\circ}$ and $220^{\circ}$. However, the possibility of other Family identification, especially secondary Families below $5^{\circ}$ or above $270^{\circ}$ (e.g., $\Delta \phi_{c}=2.5^{\circ}$ might be predicted), should not be excluded from future studies.

In many instances within Families, the separations between radials may be written in terms of $k=j / 4$ or $j / 3$ $(j=1,2,3 \ldots)$, with a preference for $k=j / 2\left(\chi^{2}=77, d f=1\right.$, $p<0.001$ and see Evidence of a Quantum Nature Subsection 5.5 for further comment), amounts of some proposed quantum increment value, $\alpha$, determined through the previously described iterative analysis procedure. Mean Family $\alpha$ values, $\bar{\alpha}_{\Delta \phi}$, form ratios with relatively precise common fraction alignment, with $\bar{\alpha}_{\Delta \phi}$ ratios classified as "Tier 1 " within table 7 including:

$\bar{\alpha}_{5^{0}}: \bar{\alpha}_{30^{0}}=3: 2(0.024 \%), \bar{\alpha}_{10^{0}}: \bar{\alpha}_{20^{0}}=5: 4(0.032 \%)$,

$\bar{\alpha}_{10^{0}}: \bar{\alpha}_{50^{0}}=4: 5(0.32 \%), \bar{\alpha}_{90^{0}}: \bar{\alpha}_{180^{0}}=1: 5(0.23 \%)$, and

$\bar{\alpha}_{10^{0}}: \bar{\alpha}_{30^{0}}=9: 7(0.018 \%)$.

Although the high precision of the above $\bar{\alpha}_{\Delta \phi}$ ratios (with bracketed alignment percentage differences based on exact median $\bar{\alpha}_{\Delta \phi}$ values) is slightly tempered by the inclusion of 95\% CI error margins (see table 7 and figure B1) which extends the maximum percentage difference to $0.5 \%$, the recurring levels of precision based on exact median values may suggest that, while $\alpha_{\Delta \phi}$ values display some variation (e.g., between subjects and clinical states), ratio alignment with common fractions often remains precise for a given EEG segment or "moment", as per individual phase wheel analysis.

The numerical symmetry of (2), which is emphasised by expressing to two significant figures, i.e., $10 \bar{\alpha}_{\Delta \phi}=2.0 \times 10^{-3} \Delta \phi_{c}^{2}-1.0 \times 10^{-1} \Delta \phi_{c}+3.0$, is mathematically consistent with the above $\bar{\alpha}_{\Delta \phi}$ ratio findings, albeit at the reduced precision specified, though such symmetry is 
obviously not a necessary requirement for the formation of such ratios. The strong inter-Family $\bar{\alpha}_{\Delta \phi}$ versus $\Delta \phi_{c}$ parabolic functionality given by (2) raises the issue of biophysical significance which is addressed within the Evidence of a Quantum Nature Subsection 5.5. Certainly, the mathematical appropriateness of parabolic modelling is championed by the fact that the $y$-minimum appears representative of a universal quantum increment value, $\bar{\alpha}_{\text {min }}=0.174019 \pm 0.000010^{\circ}$, that has a relatively high frequency of occurrence within figure C1 such that almost every radial has connectedness to $\alpha_{\min }$ and/or some $\alpha_{\Delta \phi}$. The functionality also offers mathematical explanation of statistical outcomes demonstrating a strong departure from a normal distribution of radials (e.g., $\chi^{2}=303$, $d f=12, p<0.001$ of table 3 and $P=9.8 \times 10^{-10}$ of table 4 ), since the functionality yields the tendency for $\Delta \phi$ radial alignment with/about not only $\Delta \phi_{c}$ but also with the relatively large parent set of primary radials (now explained as arising from fractional perturbations of $\alpha_{\Delta \phi}$ about $\Delta \phi_{c}$ over all Families).

In addition to the formalised $\bar{\alpha}_{\Delta \phi}$ ratio findings above, for the seven analysed phase wheels, that collectively represent a cross-section of subjects and clinical states including relatively short and long analysis times over which high-order phase relationships might occur, the ratios of sector angles within individual phase wheels also demonstrate simple common fraction alignment. The sector angle ratios $1: 1,1: 2,2: 3$ and 3:4 have the highest propensity (for a $1 \%$ alignment tolerance), with several additional examples demonstrating alignment to within a higher precision of $0.10 \%$ : $1: 1(<0.01 \%), 1: 2(0.02 \%)$ twice identified, $1: 1$ (0.04\%), 1:2 (0.04\%), $1: \sqrt{2}(0.04 \%)$ and $2: 3(<0.10 \%)$, with bracketed values giving the percentage difference magnitude from exact alignment and here the definition of simple common fraction is liberally extended to include fractions involving an integer and $\sqrt{2}$.

The formalised $\bar{\alpha}_{\Delta \phi}$ ratio findings undoubtedly contribute to the fact that, of the phase wheel sector ratios that align to within $1.0 \%$ of a common fraction, approximately $60 \%$ involve two different primary radials and approximately $70 \%$ involve across-Family radials. That is for example, if two sectors are constructed within different Families, then the ratio of the sectors' angles can be expected to form a common fraction when the sector angles are fractional amounts of quantities ( $\alpha_{\Delta \phi}$ values) that are themselves fractionally related.

The most highly represented Families within the sector ratios of interest are the $20^{\circ}$ and $30^{\circ}$ Families (approximately 50\% representation), followed by the $10^{\circ}$ and $50^{\circ}$ Families (approximately 30\% representation), followed by the $90^{\circ}$ Family. These propensities not surprisingly appear influenced by Family populations (i.e., the $20^{\circ}$ and $30^{\circ}$ Families are the most populated) which in turn are weighted by the fact that several contributing EEG segments belong to sleep subjects, and it is clear that a trend towards lower $\Delta \phi$ (and ultimately towards a linear $\phi$ versus $t^{\prime}$ relationship) exists for progressively deeper sleep stages.

The noted $1: \sqrt{2}(0.04 \%)$ sector ratio alignment of one analysed phase wheel (not shown) is accompanied by $1: \sqrt{2}(0.26 \%)$ and $1: \sqrt{2}(0.81 \%)$ sector ratio alignments from the same phase wheel. While the triple occurrence of $\sqrt{2}$ within sector ratios naturally raises interest, the occurrences must be treated as cautionary due to occurrence being within the EEG segment of one subject only. However, the finding is nonetheless flagged as an area for future verification or contradiction.

\subsection{EVIDENCE OF A QUANTUM NATURE}

\subsubsection{QUANTISED INCREMENTS AND PARABOLIC FUNCTIONALITY}

The cerebral cortex potentially represents the ultimate quantum computer. Accordingly, the manner in which $\Delta \phi$ radial separations within each Family align with $k \alpha_{\Delta \phi}$ (where $k$ is preferably an integer or half-integer over other fractional integers, $\chi^{2}=77, d f=1, p<0.001$ ) is possibly suggestive of some form of quantised neurophysiology, especially given the significance of integer and half-integer increments in many well known quantum scenarios (such as quantised principal electron orbitals and spin-based fine structure splitting). The discrete nature of the highly correlated parabolic modelling of $\bar{\alpha}_{\Delta \phi}$ versus $\Delta \phi_{c}$ for all Families (figure 9) also supports this possibility since parabolic functionality is observed across a spectrum of fundamental quantum scenarios - four example scenarios follow:

\section{Quantum parabola example 1}

Parabolic modelling of noble gas atomic numbers, $Z$, versus a generic quantum number, $x=0,1,2,3 \ldots$, yields the mathematically engaging parabolic function $Z=\left(3-\frac{1}{7}\right) x^{2}+2 x+\left(3+\frac{1}{7}\right)$, where the function's coefficients are exact (not rounded), and $y$-residuals from the model function demonstrate quantised and highly correlated structure [65]. Here, elucidation is also provided as to why the uncertainty analysis applied to the coefficients of the inter-Family parabolic modelling of figure 9 incorporates $\bar{\alpha}_{\Delta \phi}$ error margins but not standard error ( $y$-variance regression) analysis. Viz., the latter analysis assumes that residuals away from the model function represent model error. However, from a quantum mechanical perspective, perturbations from such models are typically quantised and have physical significance (e.g., commonly associated with the fine structure of atomic energy levels). Hence, it is inappropriate to effectively force data points back onto the model function and an assumption that residuals have physical significance is more appropriate for the EEG quantisation postulate at hand. 


\section{Quantum parabola example 2}

Bohr's famous model of atomic hydrogen, though considered classical or semiclassical in nature, is nonetheless based on a landmark quantisation of angular momentum postulate, and calculates the energies of principal electron orbit levels via a succinctly derived theoretical term involving $n^{-2}$, where $n$ is a principal orbital quantum number. A theoretical extension to Bohr's model derives an additional $n^{-4}$ term and allows the calculation of fine energy structure to accuracies comparable to that of relativistic Dirac theory [66]. Hence, while semiclassical approaches to quantum systems always possess limitations, Bohr's original model and its cited extension mathematically (if not physically) reflect parabolic functionality of hydrogen energy levels (after rudimentary variable substitution is applied to the original $n^{-2}$ and new $n^{-4}$ terms).

\section{Quantum parabola example 3}

A particle contained within an infinite square well potential represents one of the most fundamental quantum systems studied by students learning introductory quantum mechanics. Solutions to Schrödinger's time-independent equation for such a potential yields eigenvalues (energy solutions) that are proportional to $n^{2}$, with $n$ again representing a principal quantum number. Hence, the energy functionality is parabolic, and parallels may certainly be drawn with the containment of ions within the confines of the nerve cell membrane and under the influence of a well-like action potential.

\section{Quantum parabola example 4}

Within the quantum formalism of some atomic interactions, the total molecular potential of a formed temporary molecule often includes a centripetal term (that relates to the centripetal motion of the atoms), $V_{c}$, such that $V_{c} \propto J(J+1)$, where $J$ is a quantum number labelling the eigenvalues associated with the total molecular angular momentum. This $V_{c} \propto J(J+1)$ term can play a crucial resonance role within atomic interactions (as expanded upon and linked to conventional EEG peaks within Appendix D) and inherently displays parabolic functionality based on its second-order mathematical form.

\subsubsection{SYMMETRICAL CONSIDERATIONS}

Several phase wheels display radial symmetry and in select cases the mid-line between symmetrical radials is used to form sectors yielding angular ratios aligning with common fractions (e.g., the $\eta: \kappa$ and $\kappa: \lambda$ sector ratios of phase wheel figures $4 \mathrm{e}$ and $5 \mathrm{e}$ respectively). Additionally, symmetrical $\Delta \phi$ clustering about $\Delta \phi_{c}$ is evident to varying degrees within the figure 8 transition diagrams. Such symmetrical considerations are of interest since they allow further quantum parallels to be drawn or at least considered (e.g., parallels with the symmetries of spin-based spectral splitting and the existence of some atomic resonances in pairs [67]).

The inherent circular symmetry that comes with measuring the phase difference between any two sinusoidal harmonics validates the consideration of complementary radials within aspects of $F T^{\prime}$ analysis, and so the finding that complementary radials appear relevant for some identified Families (recall that the $\Delta \phi_{c}=135^{\circ}$ Family was relegated to secondary Family status on the basis of a too heavy reliance on complementary radials) may also be indicative of such quantum mechanical parallels. Accordingly, the above-described $\Delta \phi$-based symmetries may well be a consequence of some lower, preferred (parallel) versus higher, non-preferred (anti-parallel) neural state or process, and contribute to the volume of evidence supporting a quantum aspect to presented findings.

More generally, it is pertinent to recall that within physics every symmetry identifies with a conservation law ${ }^{3}$ and, in some common cases, it is parabolic symmetry that leads to the conservation (e.g., as classically observed for some projectile motion parameters within the confines of total mechanical energy conservation), such are the mathematical advantages and applications of the parabolic form.

\subsubsection{TRANSFORMATION TO ELEMENTAL LABELLING}

The transformation $\Delta \phi_{c} / 5 \rightarrow \mathrm{z}$ (see figure 9) yields, for primary Families in ascending order, $z=1,2,4,6,10,18$, 36 and 54. As per Subsection 4.2.3, based on an atomic number nomenclature this transformation allows the primary Families to be respectively labelled as $H\left(1 s^{1}\right)$, He $\left(1 s^{2}\right)^{*}, \operatorname{Be}\left(2 s^{2}\right), \mathrm{C}\left(2 p^{2}\right)$, Ne $\left(2 p^{6}\right)^{*}, \operatorname{Ar}\left(3 p^{6}\right)^{*}, \operatorname{Kr}\left(4 p^{6}\right)^{*}$, and $\mathrm{Xe}\left(5 \mathrm{p}^{6}\right)^{*}$, where an asterisk signifies a stable noble gas. Note that this labelling is not suggestive of elemental involvement, but of the possible involvement of some proportionally optimal quantised state within EEG neurophysiology. For $\Delta \phi_{c}=10^{\circ}$ it is interesting that $\alpha_{10^{\circ}}$

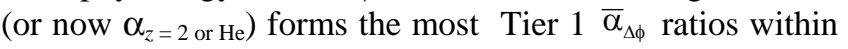
table 7 , since $\mathrm{He}$ is the highest rated universal element in terms of combined abundance and stability.

The above declaration that elemental labelling is suggestive of some proportionally optimal quantised state (or, to re-express, representative of another example of quantum mechanical forms reoccurring across many scales of the physical world as per the approximate repetition of quantised energy level structural form from the level of quarks upwards) and not actual elemental involvement, cannot be overemphasised. Available evidence does not allow further conclusion to be drawn. However, when Dmitri Mendeleev first constructed the periodic table, electrons and orbital concepts were unknown, with elements grouped by their masses and properties. Vacancies within the original periodic table were, in the

\footnotetext{
${ }^{3}$ The symmetry-conservation law association is that of mathematics' historical matriarch, Emmy Noether, as acknowledged by [68]
} 
fullness of time, filled by other scientists leading to discoveries of new elements, with the evolving table eventually paving the way for the understanding of: proton, neutron and electron arrangements; chemical reactivity; and ultimately quantum mechanics [68]. Hence, the preliminary elemental labelling finding of the present study similarly encourages developmental findings that may in time lead to cerebral cortex process understandings that perhaps have not yet been envisaged. Several other elemental labelling outcomes of curious interest exist, including elements of biological abundance and significance, but are not specified here in line with the present study's scope.

\subsubsection{QUANTUM MECHANICAL OVERVIEW}

In summary, wide-ranging quantum mechanical underpinnings of the EEG are, perhaps unsurprisingly, indicated by: radial symmetry; the quantised structure of intra-Family radial separations; parabolic inter-Family functionality; high degrees-of-freedom displayed by structured $\Delta \phi$ within the $F T^{\prime}$; the preliminary finding of elemental labelling (or proportionalities between $\Delta \phi_{c}$ and $Z$ values of stable elements); and shared characteristics between common quantum resonance types within atomic interactions and peaks within the conventional EEG frequency spectrum (addressed within Appendix D). Such quantum complexities within the cerebral cortex could fittingly, for such a profoundly sophisticated and computationally powerful entity, be described as biological quantum entanglement which only a quantum treatment of the EEG can fully interpret into the future.

\subsection{OTHER FUTURE CLINICAL RESEARCH DIRECTIONS AND IMPLICATIONS}

\subsubsection{DECIPHERING OF FT' ENCODING}

While the present study reveals considerable mathematical detail in regards to the manner of $F T^{\prime}$ encoding based on upward $\Delta \phi$ analysis, scope clearly remains for $F T^{\prime}$ deciphering in a clinical context, including with additional downward and/or horizontal $\Delta \phi$ analysis. Such deciphering studies, e.g., that quantitatively investigate the manner of deliberate $\Delta \phi$ management and coordination for specific CNS function and stimuli (perhaps even emotional), have the potential to significantly contribute to BCI and neuroprosthetic interfacing advancements.

The similarity of the two (prefrontal and central) linear but slightly oscillatory $\phi$ versus $t^{\prime}$ profiles of figure $6 g$, reflecting origin source overlap, is not surprising and consistent with coherence and range-based correlation findings of conventional EEG frequency analysis [24], as well as with the Subsection 4.1.1 finding of a generally similar outcome for $F T^{\prime}$ amplitude profile shape when comparing the averages of EEG channels 1 to 12 against channels 13 to 19 . However, such profile comparisons may also offer opportunity for more specific origin and functional connectivity information identification due to the profiles containing the phases of many $F T^{\prime}$ harmonics. That is, the two profiles under comparison in figure $6 \mathrm{~g}$ are mostly identical except for subtle $\phi$ variances (that may still maintain the same $\Delta \phi$ in some instances as demonstrated by figure $6 \mathrm{~g}$ ), and so harmonic propagation time or amplitude comparisons (e.g., degree of delay, attenuation, etc.) on a harmonic-by-harmonic basis for many profiles may provide opportunity for mathematical reconstruction (e.g., via a forward model) that reveals highly specific spatiotemporal origin distribution and functional connectivity information.

\subsubsection{INCREASING LINEARITY WITH INCREASING SLEEP DEPTH FOR THE FT'}

A full clinical study that investigates linearity and $d \phi / \mathrm{d} t^{\prime}$ of $\phi$ versus $t^{\prime}$ as potential indices of sleep stage naturally derives from the present study. Likewise, the sensitivities of these potential indices towards depth of anaesthesia, awake vigilance, and consciousness in general, are also indicated as topics of future clinical research. An additional aspect of such future research could further quantify the sleep transition characteristics of $F T^{\prime}$ amplitude profile form, with the present study indicating dynamically changing amplitude profiles that include the display of $e^{-t^{-x} / \tau}$ behaviour.

\subsubsection{THE SENSITIVITY OF FT' TOWARDS NEUROPATHOLOGY}

The two $F T^{\prime}$ spectra for an elderly probable Alzheimer's subject (figures 5b and 5d) produced several examples of the types of common fraction ratios and structured $\Delta \phi$ radial separations of interest within the present study. However, notable is the contrasting comparison between figures $4 \mathrm{~d}$ and 5d (healthy adult versus probable Alzheimer's respectively for prefrontal EEG with eyes-closed). The healthy adult spectrum displays $d \phi / \mathrm{d} t^{\prime}$ approaching (approximate) early constancy and the mostly featureless spectrum is consistent with the intuitive expectations of a relatively neutral neural state. The probable Alzheimer's spectrum contrastingly displays, for similarly desired neural neutrality, a full (almost "frantic") spectrum of $\Delta \phi$ that is comparable to the subject's simultaneous occipital spectrum (figure 5b). Also, the oscillatory behaviour of the $F T^{\prime}$ amplitude profile for the healthy adult in figure $4 \mathrm{~b}$ is not apparent within the probable Alzheimer's counterpart figure 5b. Despite marked alpha activity differences which are clearly influential, these comparisons could indicate a sensitivity of the $F T^{\prime}$ spectrum towards various neurological disorders. Hence, a large-scale study of healthy versus neuropathological $F T^{\prime}$ would constitute a meaningful future research direction and potentially contribute to the ongoing understanding of such disorders. 


\subsubsection{THERAPEUTIC INTERVENTIONS (INCLUDING MUSIC THERAPY) TO FACILITATE HEALTHY $\Delta \phi$ MODULATION OF THE FT'}

Subsection 5.3 rationalised the oscillatory nature of $F T^{\prime}$ spectra as being deliberate. However, the added evidential dimension of $F T^{\prime}$ oscillatory behaviour being ratio-based may also rationalise a connectedness to music-based neurophysiological research, which for example has found: (i) a strong association between rhythm (perception skills) and language development in young children that is linked to the sharing of neural networks/cognitive resources between rhythm and grammatical structure learning [69]; (ii) that listening to classical music (by musically experienced participants) enhances the activity of genes responsible for brain function (in particular genes involved with dopamine secretion and transport, synaptic neurotransmission, learning and memory), with the synuclein-alpha gene (a known risk gene for Parkinson's disease) being one of the most enhanced [9]; (iii) benefits of music therapy for the treatment of dementia-based behavioural disorders [70]; and more generally (iv) multiple effects, both structural and functional, on the cerebral cortex in response to deliberate music exposure $[6,7,9]$ (these examples represent only a small subset of a profusion of music-based neurophysiological research).

The identification of common fraction-aligned $\bar{\alpha}_{\Delta \phi}$ ratios, as well as common fraction-aligned sector angle ratios for sectors formed by $\Delta \phi$ radials within individual phase wheels, potentially contributes to the understanding of the biophysical determinants that underlie music therapy/exposure (these determinants remain largely unknown despite the above-noted research profusion [9]). Viz., Tier $1 \bar{\alpha}_{\Delta \phi}$ ratios align, with high precision, to $3: 2$, 5:4, 4:5, 1:5 and 9:7, and sector ratios with the highest propensity within individual phase wheels align, with high precision, to $1: 1,1: 2,2: 3$ and $3: 4$, and all of these ratios combined (except 9:7) are prominent within (the frequency interval proportions of) pentatonic music scales ${ }^{4}$ that are accepted as being the most historically significant scales on the basis of their independent development by many ancient cultures [71,72]. Several other examples of connectedness between key ratios of the present study and those that are fundamental to music theory and formalisms (e.g., in the area of timing and rhythm) also exist but are not specified here in line with the present study's scope.

Hence, future research that involves the delivery of therapeutic alleviatory interventions via music, TMS, or

\footnotetext{
${ }^{4}$ Pentatonic music scales divide the octave into fifths (i.e., five notes per octave). These scales naturally involve the ratio $2: 1$, since it is fundamental to the octave concept, as well as the ratio 5:1 given the scales' divisional methodology. Additionally within pentatonic scales, the ratio 3:2 is referred to as the "pure perfect fifth" and the Pythagorean pentatonic scale (which overlaps with other pentatonic scales) is constructed solely from these $3: 2$ and 2:1 ratios, giving the following fundamentally important tunings: 5:4 major third; $4: 3$ perfect fourth; $3: 2$ perfect fifth; 2:1 perfect octave or duplex; and 1:1, 5:1 (inherently included for reasons stated).
}

other means, and designed to stimulate or enhance healthy neurological function (e.g., by resonant absorption of an applied therapeutic medium containing appropriate ratiobased modulations or modulations of a time-domain waveform constructed on the basis of other ideal $F T^{\prime}$ characteristics), and which involves pre- and post-therapy comparisons with healthy EEG, therefore appears warranted. Furthermore, the small 95\% CI error margins of $\bar{\alpha}_{\text {min }}$ and $\bar{\alpha}_{\Delta \phi}$ values (equating to percentage errors ranging from 0.006 to $0.39 \%$ with an average of $0.19 \%$ ), appear to indicate that the values of $\alpha$-related quantities will be tightly confined across a wider population, and thus any future therapies that are found to be beneficial by specifically targeting these or associated quantities may to some extent not require individual prescriptions in terms of applied modulation settings.

\subsubsection{IMPLICATION OF POSSIBLE ZOOLOGICAL UNIFORMITY}

An interesting zoological offshoot finding is that Tier $1 \bar{\alpha}_{\Delta \phi}$ ratios coincide with dominant ratios $(3: 2,5: 1)$ of the most used sperm whale communication (click-based) codas [73]. This offshoot finding is raised because, for many, sperm whales represent the largest cooperative group of vertebrates outside of humans, and have a multilevel social system with an advanced, culturally transmitted communication system [73-75]. A ratio-based finding can also be extrapolated from communication research for Beluga whales [76], known for being highly vociferous and generating a variety of calls using tonal and pulsed components with an added ability to copy human speech (in a limited manner). Ratios of average inter-pulse intervals (interval at pulse train end:interval at pulse train beginning) within the dominant broadband pulse sound (PS1 call) of isolated whales display the "quintuple" ratios 5:4, 7:5 and 6:5. Note however that, unlike the initial sperm whale example, this extrapolated result is preliminary.

Hence, a finding that communication encoding methods for the Earth's most intelligent species share the same dominant ratios encourages contemplations on several levels and, as per the ratios-in-music coincidence findings, arguably adds support to the neurological significance (and thus mathematical mechanism and clinical efficacies) of the present study's technique, in turn providing additional motivation for the future clinical research directions advocated within Subsection 5.6.

\section{CONCLUSION}

Based on the introduced high-order phase analysis technique, it is concluded that the EEG is encoded by quantised phase transitions between newly-identified harmonics across the conventional frequency spectrum, allowing for powerful phase-shift keying impulse encoding complexity with high transitional degrees-of-freedom. The technique commences with the double application of high resolution Fourier analysis applied to the EEG, leading 
to $F T^{\prime}$ spectra (that include profiles of new harmonic phase, $\phi$, over an effective time-domain, $\left.t^{\prime}\right)$. The $\phi$ versus $t^{\prime}$ behaviour of such profiles generally becomes increasingly linear with the progression of neural state towards deep sleep. Three separate stage 4 sleep analyses (i.e., on three subjects) involving 17 EEG segments display high linear correlations $(-r \geq 0.990, p<0.001$ in all 17 cases) with regression rates, $d \phi / \mathrm{d} t^{\prime}$, ranging from -163 to $-236^{\circ} \cdot \mathrm{s}^{-1}$ and on average displaying error margins of $\pm 3 \%$ (95\% CI). Such linearities are in stark contrast to the chaotic-like nature of harmonic phase for conventional EEG frequency analysis (first-order analysis), and chaotic elements of established phase-based analysis techniques such as second-order bispectral analysis.

For awake and stages 1 to 3 sleep, $F T^{\prime}$ spectra display many structured $\phi$ transitions, including oscillatory behaviour in response to peaks (e.g., alpha, sigma sleep spindle) within the conventional frequency spectrum. For transitions whereby $\phi$ increases by $\Delta \phi$ between local minima and maxima, the combined $\Delta \phi$ values from seven $F T^{\prime}$ spectra (that include pathology, electrode placement and analysis time diversity), are found to cluster rather than follow a normal distribution (e.g., $\chi^{2}=303, d f=12, p<$ $0.001)$. Influential $\Delta \phi$ clustering is categorised into ten "Families", with primary Families displaying clustering about $\Delta \phi_{c}=5^{\circ}, 10^{\circ}, 20^{\circ}, 30^{\circ}, 50^{\circ}, 90^{\circ}, 180^{\circ} 270^{\circ}$ and secondary Families displaying clustering about $\Delta \phi_{c}=135^{\circ}$ and $220^{\circ}$.

In many instances within Families, the separations between $\Delta \phi$ values are highly structured and may be written in terms of $k=j / 4$ or $j / 3(j=1,2,3 \ldots)$, with a preference for $k=j / 2\left(\chi^{2}=77, d f=1, p<0.001\right)$, amounts of some proposed quantum increment value, $\alpha$, such that separation is given by $k \alpha$. The $\alpha$ value for each Family, $\alpha_{\Delta \phi}$, is extracted as the gradient of $\Delta \phi$ separation versus $k$ multiplier in an iterative (separation acceptance/rejection) manner that applies $r>0.9999, p<0.001$ thresholds for Family identification. The 95\% CI mean values, $\bar{\alpha}_{\Delta \phi}$, for the primary Families are:

$\bar{\alpha}_{5^{0}}=0.26534 \pm 0.00092^{\circ}, \bar{\alpha}_{10^{0}}=0.22734 \pm 0.00062^{\circ}$,

$\bar{\alpha}_{20^{0}}=0.18193 \pm 0.00023^{\circ}, \bar{\alpha}_{30^{0}}=0.17685 \pm 0.00007^{\circ}$,

$\bar{\alpha}_{50^{0}}=0.28509 \pm 0.00019^{\circ}, \bar{\alpha}_{90^{0}}=0.98495 \pm 0.00033^{\circ}$,

$\bar{\alpha}_{180^{0}}=4.9134 \pm 0.0116^{\circ}$ and $\bar{\alpha}_{270^{\circ}}=11.981 \pm 0.040^{\circ}$,

with values for the secondary Families being $\bar{\alpha}_{135^{0}}=2.5329 \pm 0.0055^{\circ}$ and $\bar{\alpha}_{220^{\circ}}=7.7336 \pm 0.0302^{\circ}$.

Family $\bar{\alpha}_{\Delta \phi}$ versus $\Delta \phi_{c}$ values display strong parabolic functionality $(r=0.99998, p<0.001)$ given by $\bar{\alpha}_{\Delta \phi}=$ $(1.9772 \pm 0.0049) \times 10^{-4} \Delta \phi_{c}^{2}-(9.990 \pm 0.097) \times 10^{-3} \Delta \phi_{c}$

$+(3.002 \pm 0.021) \times 10^{-1} \quad(95 \% \mathrm{CI})$, with a universal quantum increment value, $\alpha_{\text {min }}$, spanning across Families and equating to the turning point (minimum) of the parabolic function, given by $\bar{\alpha}_{\min }=0.174019 \pm 0.000010^{\circ} . \bar{\alpha}_{\Delta \phi}$ values form ratios with relatively precise common fraction alignment and these ratios, when classified as "Tier 1" (involving primary Families and simple common fractions), include:

$\bar{\alpha}_{5^{0}}: \bar{\alpha}_{30^{0}}=3: 2(0.024 \%), \bar{\alpha}_{10^{0}}: \bar{\alpha}_{20^{0}}=5: 4(0.032 \%)$,

$\bar{\alpha}_{10^{0}}: \bar{\alpha}_{50^{0}}=4: 5(0.32 \%), \bar{\alpha}_{90^{0}}: \bar{\alpha}_{180^{0}}=1: 5(0.23 \%)$, and

$\bar{\alpha}_{10}: \bar{\alpha}_{30^{\circ}}=9: 7(0.018 \%)$, with bracketed values giving the percentage difference between the actual formed ratio and its nominal common fraction representation. Although a case exists for basing these ratios on exact (median) $\bar{\alpha}_{\Delta \phi}$ values, all percentage differences nevertheless remain within 0.5\% when 95\% CI error margins are included. Several Tier 2 and 3 ratios, with classifications explained within, are also identified.

For individual subjects, $\Delta \phi$ values for any given $F T^{\prime}$ spectrum often display symmetries and form sectors within polar diagrams (phase wheels), with the ratios of sector angles often aligning with high precision to common (and other) fractions. Identified sector ratios from seven phase wheels of diversity and to within $0.1 \%$ alignment include: $1: 1$ (<0.01\%), 1:1 (0.04\%), $2 \times 1: 2$ (0.02\%), 1:2 (0.04\%), $2: 3(<0.10 \%)$, and $1: \sqrt{2}(0.04 \%)$. When the alignment threshold is raised to $1.0 \%$, the sector ratios with the highest propensity include 1:1, 1:2, 2:3 and 3:4.

Given the overlapping common ratio-based nature of music (e.g., fundamental ratios of the culturally and historically important pentatonic scale contain the sector ratios of propensity and all but one Tier 1 ratio) and the profusion of research into the neural effects of music exposure, a possible linkage of the present study's findings to the biophysical determinants of such effects is indicated.

The symmetrical, quantised structure of dependent Family $\alpha_{\Delta \phi}$ and $\Delta \phi_{c}$ values, the consistency of this structure's parabolic functionality with functionalities of well known quantised systems, and the linkage of conventional EEG frequency peaks to quantum mechanical resonances, together suggest a quantum mechanical governance to neural impulse generation. An additional preliminary but nonetheless intriguing offshoot quantisation finding includes the linear transformation of primary Family $\Delta \phi_{c}$ values, via simple proportionate mapping, to the atomic numbers of periodic table elements of note, resulting in respective primary Family elemental labelling of $\mathrm{H}, \mathrm{He}^{*}$, $\mathrm{Be}, \mathrm{C}, \mathrm{Ne}^{*}, \mathrm{Ar}^{*}, \mathrm{Kr}^{*}$ and $\mathrm{Xe}^{*}$ (where the asterisk signifies a noble gas), and suggestive of neuro-quantum processes involving proportionally optimal quantum states. A need for a fully quantum treatment of the EEG is subsequently indicated.

The fine impulse encoding details ( $\phi$-based unisons) extracted with confidence from a seemingly modest EEG set supports the present study's position that vast amounts of accessible information currently goes unrecognised by standard EEG analysis and consequently several clinical studies based on the introduced analysis technique are proposed for the future. These unisons will also provide insights for future BCI and neuroprosthetics research. 


\section{APPENDIX A - VALUES OF SELECT $\triangle \phi$ AND EXAMPLE PHASE WHEEL SECTOR ANGLES}

Table A1 gives horizontal and vertical (upward) $\Delta \phi$ (rounded to one decimal place) pertaining to the annotations of figures $4 \mathrm{~b}, 5 \mathrm{~b}$ and $5 \mathrm{~d}$, and aligned common fraction radian equivalent for alignment margins that typically fall within $\pm 0.5^{\circ}$ unless indicated otherwise. Labels such as " $90^{\circ} \mathrm{a}$ " indicates the local maximum labeled " $90^{\circ}$ " and adjacent to the vertical transition labeled "a", and so on.

Table A2 presents selected sector angle values for the example phase wheels of figures $4 \mathrm{e}, 5 \mathrm{e}$ and $5 \mathrm{f}$. For presentation purposes within this table, angles are rounded to four significant figures (and so minor differences with sector ratio common fraction alignment values presented in tables 1 and 2 may occur).

Table A2 demonstrates a theme of recurring quantised angular increments, consistent with other results presented throughout. In the phase wheel of figure $4 \mathrm{e}, \kappa$ (based on mid-line radial symmetry) may be precisely expressed in terms of the figure $9 y$-turning point (minimum) value, $\alpha_{\text {min }}$ (see Appendix C), giving $\kappa=10 \alpha_{\min }(0.25 \%)$. Similar outcomes (sector angles based on radial symmetry and being precise integer multiples of $\alpha_{\min }$ ) are observed within two other (not shown) phase wheels, giving $50 \alpha_{\text {min }}$ $(0.048 \%)$ and $10 \alpha_{\min }(0.22 \%)$ respectively, and thus acrossphase wheel ratios of 1:5:1 based upon these angles.

Table A2. Select sector angles for example phase wheels.

\begin{tabular}{|l|l|l|}
\hline \multicolumn{3}{|c|}{ Select sector angle (radians) } \\
\cline { 1 - 1 } \multicolumn{1}{|c|}{ figure 4e } & \multicolumn{1}{c|}{ figure 5e } & \multicolumn{1}{c|}{ figure 5f } \\
\cline { 1 - 1 }$\eta=0.01995$ & $\xi=0.014985$ & $\eta=0.008884$ \\
$\kappa=0.03030$ & $\chi=0.02042$ & $\gamma=0.005917$ \\
$v=4 \times 0.01990$ & $\varphi=2 \times 0.02048$ & $\chi=0.01965$ \\
$\tau=4 \times 0.02207$ & $\eta=5 \times 0.02037$ & $\kappa=2 \times 0.01993$ \\
$\rho=4 \times 0.02428$ & $\mu=5 \times 0.03082$ & $\lambda=0.021363$ \\
& $\gamma=3 \times 0.02149$ & $\varphi=0.02261$ \\
& $\alpha=4 \times 0.02121$ & $\rho=2 \times 0.02262$ \\
& $\beta=6 \times 0.02149$ & $\mu=2 \times 0.02248$ \\
& $v=3 \times 0.019641$ & $\beta=0.032306$ \\
& & $\alpha=0.03283$ \\
&
\end{tabular}

Table A1. Horizontal and vertical (upward) $\Delta \phi$ (rounded) pertaining to the annotations of figures $4 b, 5 b$ and $5 d$.

\begin{tabular}{|c|c|c|c|c|c|}
\hline Vertical & $\begin{array}{c}\Delta \phi \\
\text { (deg) }\end{array}$ & $\begin{array}{c}\Delta \phi \\
\text { (rad) }\end{array}$ & Horizontal & $\begin{array}{c}\Delta \phi \\
\text { (deg) }\end{array}$ & $\begin{array}{c}\Delta \phi \\
\text { (rad) }\end{array}$ \\
\hline \multicolumn{6}{|c|}{ figure $4 b$} \\
\hline $\mathrm{a}$ & 105.4 & $(1 / 3+1 / 4) \pi$ & $90^{\circ} a-60^{\circ}$ & 30.0 & $1 / 6 \pi$ \\
\hline C & 149.4 & $5 / 6 \pi$ & $90^{\circ} a-30^{\circ}$ & 59.7 & $1 / 3 \pi$ \\
\hline d & 180.1 & $\pi$ & $90^{\circ} a-45^{\circ}$ & 44.9 & $1 / 4 \pi$ \\
\hline e & 180.6 & $\approx \pi$ & $60^{\circ}-30^{\circ}$ & 29.8 & $1 / 6 \pi$ \\
\hline $\mathrm{f}$ & 10.0 & $1 / 18 \pi$ & $60^{\circ}-45^{\circ}$ & 15.0 & $1 / 12 \pi$ \\
\hline g & 4.6 & $1 / 36 \pi$ & $60^{\circ}-20^{\circ}$ & 39.8 & $2 / 9 \pi$ \\
\hline $\mathrm{i}$ & 22.4 & $1 / 8 \pi$ & $30^{\circ}-20^{\circ}$ & 10.1 & $1 / 18 \pi$ \\
\hline $\mathrm{j}$ & 5.1 & $1 / 36 \pi$ & $30^{\circ}-25^{\circ}$ & 5.0 & $1 / 36 \pi$ \\
\hline $\mathrm{k}$ & 19.8 & $1 / 9 \pi$ & $45^{\circ}-25^{\circ}$ & 19.8 & $1 / 9 \pi$ \\
\hline $\mathrm{b}-\mathrm{a}$ & 140.5 & $7 / 9 \pi$ & $25^{\circ}-20^{\circ}$ & 5.1 & $1 / 36 \pi$ \\
\hline$c-a$ & 44.0 & $\approx 1 / 4 \pi$ & & & \\
\hline $\mathrm{b}-\mathrm{h}$ & 178.1 & $\approx 2 \pi$ & & & \\
\hline $\mathrm{d}-\mathrm{c}$ & 30.7 & $\approx 1 / 6 \pi$ & & & \\
\hline \multicolumn{6}{|c|}{ figure $5 b$} \\
\hline $\mathrm{a}$ & 49.8 & $5 / 18 \pi$ & $-50^{\circ}+75^{\circ}$ & 24.9 & $5 / 36 \pi$ \\
\hline b & 115.6 & & $5^{\circ}+30^{\circ} \mathrm{f}$ & 35.1 & $7 / 36 \pi$ \\
\hline C & 82.8 & & $5^{\circ}+45^{\circ}$ & 50.2 & $5 / 18 \pi$ \\
\hline d & 172.7 & & $5^{\circ}+75^{\circ}$ & 80.7 & $\approx 4 / 9 \pi$ \\
\hline e & 32.7 & & $-30^{\circ} e+50^{\circ}$ & 19.9 & $1 / 9 \pi$ \\
\hline $\mathrm{f}$ & 134.9 & $3 / 4 \pi$ & $-30^{\circ} e+75^{\circ}$ & 44.8 & $1 / 4 \pi$ \\
\hline g & 102.35 & & $-30^{\circ} \mathrm{f}+75^{\circ}$ & 45.6 & $\approx 1 / 4 \pi$ \\
\hline $\mathrm{h}$ & 91.2 & & $-45^{\circ}+75^{\circ}$ & 30.5 & $1 / 6 \pi$ \\
\hline $\mathrm{i}$ & 254.8 & & $180^{\circ}+50^{\circ}$ & 230.0 & \\
\hline $\mathrm{j}$ & 333.7 & & $180^{\circ}+75^{\circ}$ & 254.9 & \\
\hline $\mathrm{k}$ & 145.9 & & & & \\
\hline $\mathrm{i}-\mathrm{f}$ & 119.9 & $2 / 3 \pi$ & & & \\
\hline $\mathrm{k}-\mathrm{b}$ & 30.3 & $1 / 3 \pi$ & & & \\
\hline$d-c$ & 90.1 & $\pi$ & & & \\
\hline $\mathrm{c}-\mathrm{e}$ & 49.9 & $5 / 18 \pi$ & & & \\
\hline$g-c$ & 19.7 & $1 / 9 \pi$ & & & \\
\hline $\mathrm{j}-\mathrm{e}$ & 301.0 & $\approx-1 / 3 \pi$ & & & \\
\hline$j-d$ & 161.0 & $\approx 8 / 9 \pi$ & & & \\
\hline $\mathrm{j}-\mathrm{i}$ & 78.9 & $\approx 4 / 9 \pi$ & & & \\
\hline$j-f$ & 198.8 & $\approx 10 / 9 \pi$ & & & \\
\hline \multicolumn{6}{|c|}{ figure $5 d$} \\
\hline $\mathrm{a}$ & 4.8 & $1 / 36 \pi$ & $115^{\circ}+50^{\circ}$ & 164.8 & $(3 / 4+1 / 6) \pi$ \\
\hline b & 80.5 & $4 / 9 \pi$ & $115^{\circ}-45^{\circ}$ & 69.5 & $14 / 36 \pi$ \\
\hline c & 234.4 & $13 / 10 \pi$ & $115^{\circ}-0^{\circ}$ & 114.7 & $(3 / 4-1 / 9) \pi$ \\
\hline d & 200.5 & $10 / 9 \pi$ & $115^{\circ}-40^{\circ}$ & 74.6 & $15 / 36 \pi$ \\
\hline e & 29.8 & $1 / 6 \pi$ & $90^{\circ}+50^{\circ}$ & 140.2 & $7 / 9 \pi$ \\
\hline $\mathrm{f}$ & 10.0 & $1 / 18 \pi$ & $90^{\circ}-45^{\circ}$ & 45.0 & $1 / 4 \pi$ \\
\hline g & 58.9 & $\approx 1 / 3 \pi$ & $90^{\circ}-0^{\circ}$ & 90.1 & $1 / 2 \pi$ \\
\hline $\mathrm{h}$ & 118.8 & $\approx 2 / 3 \pi$ & $90^{\circ}-30^{\circ}$ & 59.7 & $1 / 3 \pi$ \\
\hline $\mathrm{i}$ & 141.85 & $\approx 7 / 9 \pi$ & $90^{\circ}-40^{\circ}$ & 50.0 & $5 / 18 \pi$ \\
\hline $\mathrm{j}$ & 182.3 & $\approx \pi$ & $45^{\circ}-0^{\circ}$ & 45.2 & $1 / 4 \pi$ \\
\hline $\mathrm{j}-\mathrm{i}$ & 40.4 & $2 / 9 \pi$ & $45^{\circ}-30^{\circ}$ & 14.8 & $1 / 12 \pi$ \\
\hline & & & $45^{\circ}-40^{\circ}$ & 5.0 & $3 / 36 \pi$ \\
\hline & & & $0^{\circ}+50^{\circ}$ & 50.1 & $5 / 18 \pi$ \\
\hline & & & $30^{\circ}+50^{\circ}$ & 80.5 & $4 / 9 \pi$ \\
\hline & & & $40^{\circ}+50^{\circ}$ & 90.2 & $1 / 2 \pi$ \\
\hline & & & $40^{\circ}-0^{\circ}$ & 40.1 & $2 / 9 \pi$ \\
\hline & & & $40^{\circ}-30^{\circ}$ & 9.7 & $1 / 18 \pi$ \\
\hline
\end{tabular}




\section{APPENDIX B - CONFIDENCE INTERVAL \\ ANALYSIS FOR TIER $1 \alpha_{\Delta \phi}$ RATIOS}

Figure B1 gives the percentage difference between actual and nominal Tier $1 \bar{\alpha}_{\Delta \phi}$ ratio values of table 7 with CIs ranging from 80 to $95 \%$ for (a) $\bar{\alpha}_{5^{0}}: \bar{\alpha}_{30^{0}}$, (b) $\bar{\alpha}_{10^{0}}: \bar{\alpha}_{20^{0}}$, (c) $\bar{\alpha}_{10^{0}}: \bar{\alpha}_{50^{0}}$, (d) $\bar{\alpha}_{90^{0}}: \bar{\alpha}_{180^{0}}$ and (e) $\bar{\alpha}_{10^{\circ}}: \bar{\alpha}_{30^{\circ}}$. In each figure the nominal ratio is given in the top left corner and the central horizontal line represents the percentage difference based on median (exact) $\bar{\alpha}_{\Delta \phi}$ values.

(b)

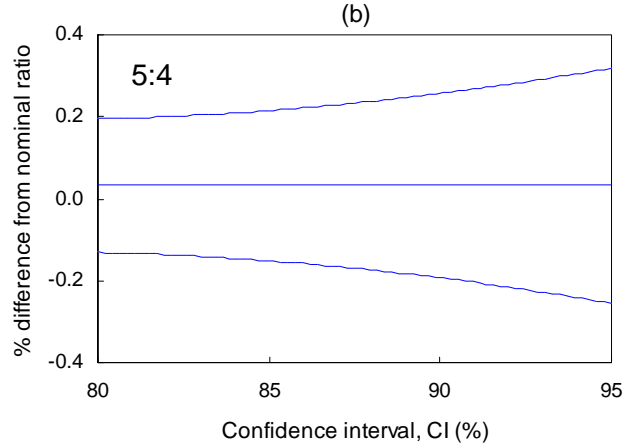

(d)

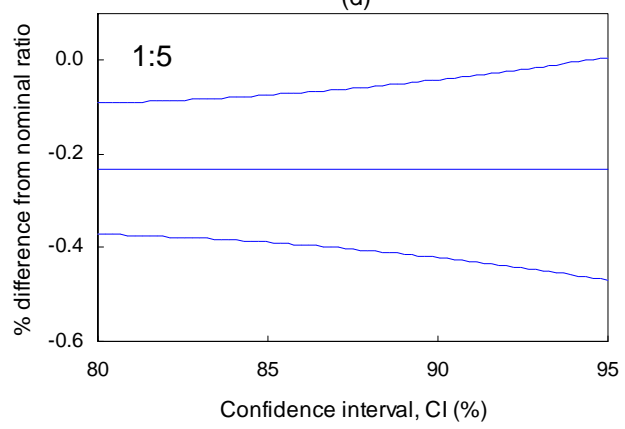

(a)

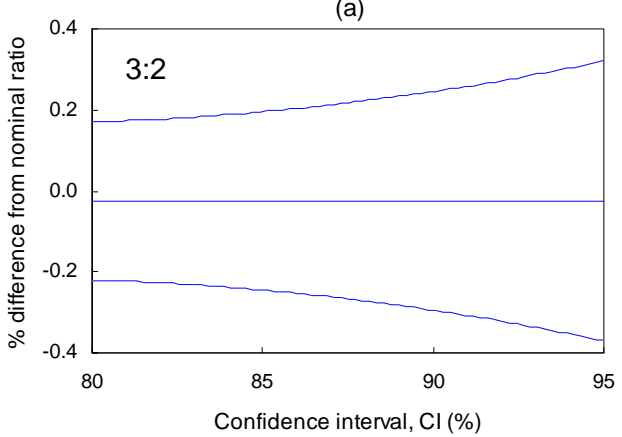

(c)

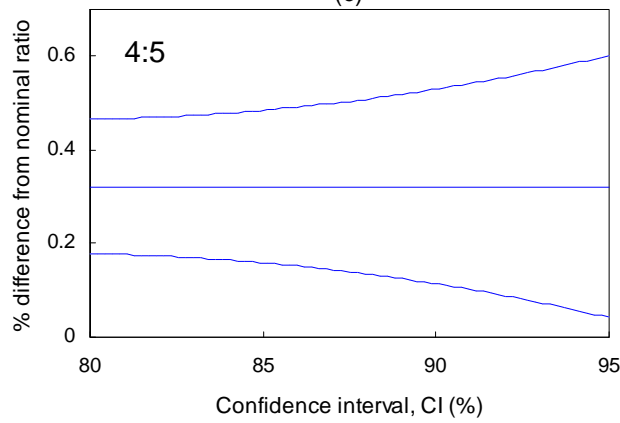

(e)

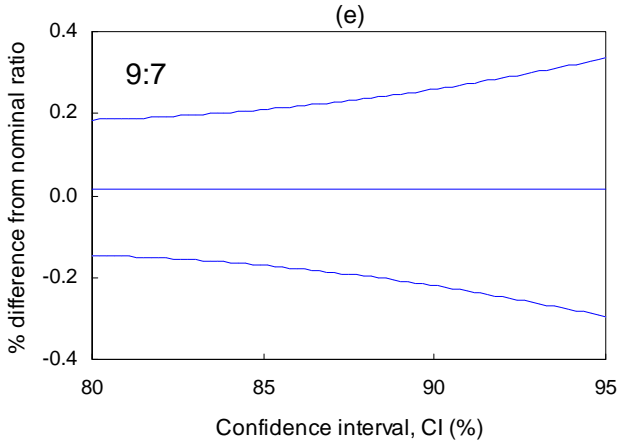

FIGURE B1. Confidence interval analysis for Tier $1 \bar{\alpha}_{\Delta \phi}$ ratio values. 


\section{APPENDIX C - RADIAL SEPARATIONS THAT}

\section{ALIGN WITH ko MIN}

Separations between $\Delta \phi$ radials that align with $k \alpha_{\min }$ are shown in figure C1 for $\alpha_{\min }=0.174019 \pm 0.000054^{\circ}$ (95\% CI). $\alpha_{\min }$ is determined by the iterative linearization process of the graphical inset and is assumed equal to the $y$-minimum of figure 9. The percentage difference between actual and nominal $\left(k \alpha_{\min }\right)$ separation values is bracketed and the percentage difference (magnitude) averaged over all separations is less than $0.2 \%$.
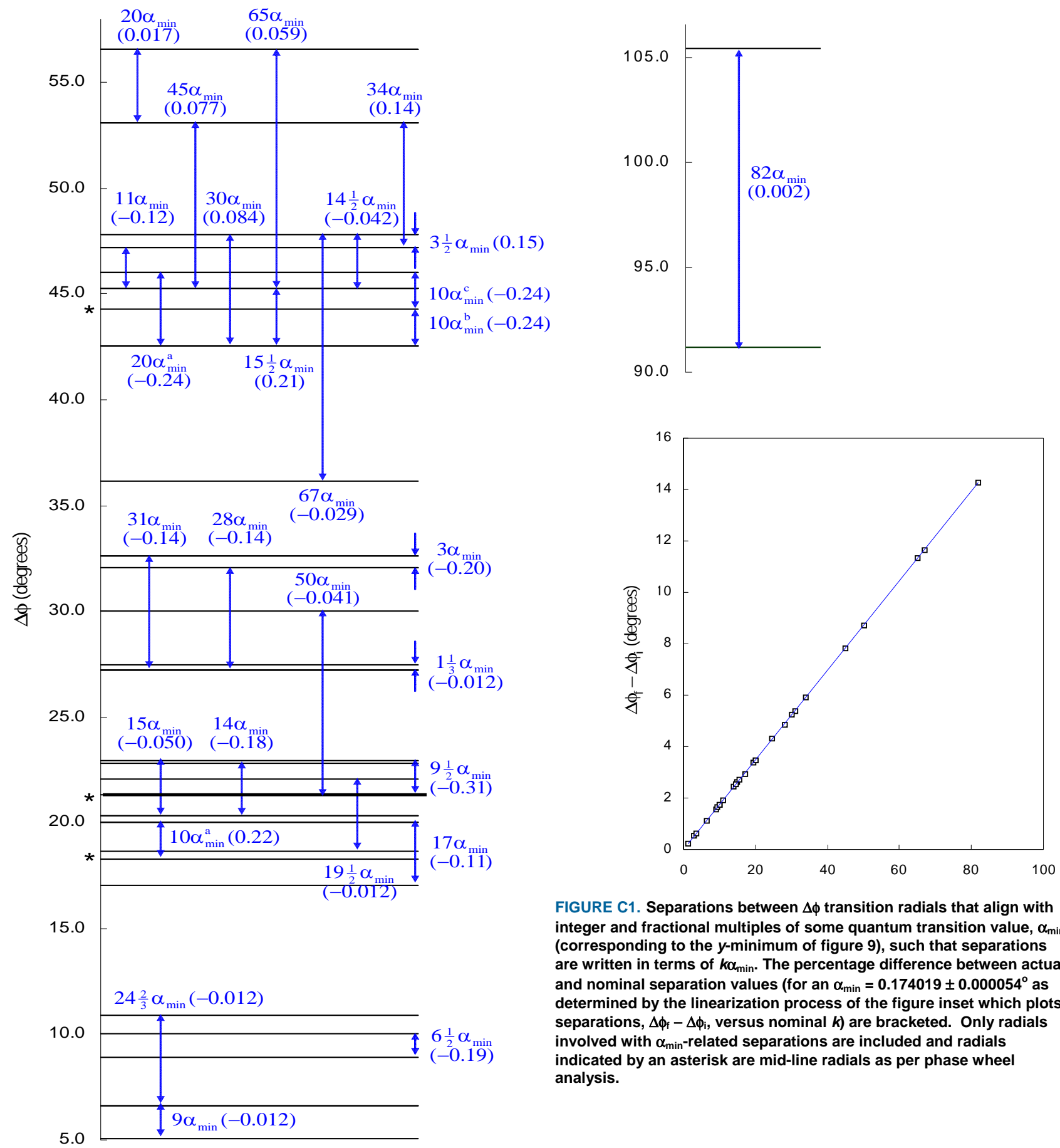

FIGURE C1. Separations between $\Delta \phi$ transition radials that align with integer and fractional multiples of some quantum transition value, $\alpha_{\text {min }}$ (corresponding to the $y$-minimum of figure 9), such that separations are written in terms of $k \alpha_{\text {min }}$. The percentage difference between actual and nominal separation values (for an $\alpha_{\min }=0.174019 \pm 0.000054^{\circ}$ as determined by the linearization process of the figure inset which plots separations, $\Delta \phi_{f}-\Delta \phi_{i}$, versus nominal $k$ ) are bracketed. Only radials involved with $\alpha_{\min }$-related separations are included and radials indicated by an asterisk are mid-line radials as per phase wheel analysis. 


\section{APPENDIX D - CONVENTIONAL EEG PEAKS CONSIDERED AS QUANTUM RESONANCES}

The quantised nature of several key findings of the present study intimates a capacity for alpha and other peaks within a conventional EEG frequency spectrum (other than perhaps those without complexity by deriving from the simplest of time-domain oscillations) to be explained as resonant quantum mechanical phenomena. This capacity is now elaborated upon by highlighting several characteristic similarities between $F T^{\prime}$ spectra and the quantum resonances of optical interactions.

Within a field such as quantum mechanics a resonance peak of some frequency spectrum is deconstructed and well understood, with quantum resonance literature spanning decades and presenting solid mathematical and physical underpinnings of such phenomena. For example, a 1973 theoretical study and review [77] associates the energy resonances of quantum systems with the exact complex poles of an appropriate Green's function (sophisticated EEG simulation models also write scalp potentials in terms of a Green's function that describes the conductive head volume [4]).

Another example of quantum resonances being well understood is found within a more recent and substantive review [78] of resonance phenomena that are particularly important for two-body atomic and molecular interactions (e.g., optical and radiative collisions). The theoretical formalisms of such interactions often depict the interacting atoms as forming a temporary molecule whereby a transition is seen to occur between initial and final molecular states as a consequence of some inputted transition-completing energy (e.g., collisional and applied laser energies). Two resonance types that readily appear when solving Schrödinger-based differential equations describing such two-body interactions are shape and Feshbach resonances.

Shape resonances are attributed to the shape of the governing molecular potential that produces quasibound states in a governing potential well; they are oscillatory and have been associated with a rapid increase in the phase shift (that occurs through odd multiples of $90^{\circ}$ ) of scattering amplitudes. Feshbach resonances are attributed to the resonances between closed and open channels ${ }^{5}$, or alternatively are described as being made possible by the presence of a molecular bound state that is reasonably coupled to states (of different symmetry and form) of the colliding atom pair. Depending on subtype, Feshbach resonances are either magnetically or optically tuneable in respect to inputted energy [78-85].

A contextually noteworthy interaction affected by such resonances is the laser assisted charge transfer (LACT) process, $\mathrm{H}^{+}+\mathrm{Na}(3 \mathrm{~s})+$ laser energy $\rightarrow \mathrm{H}(n=2)+\mathrm{Na}^{+}$[86],

\footnotetext{
${ }^{5}$ Channels are fragmentation modes of the collision's composite system with open channels allowed under known conservation laws such as the conservation of energy [81].
}

abbreviated as $\mathrm{H}^{+}$-Na. This reaction is noteworthy because the action potential that generates the EEG analogously represents a governing well-shaped potential of a molecular system undergoing charge (ion) transfer with the assistance of inputted (cellular rather than laser) energy (of course for the action potential ion transfer occurs from extracellular fluid rich in $\mathrm{Na}^{+}$and $\mathrm{Cl}^{-}$ions to intracellular fluid rich in $\mathrm{K}^{+}$ ions via transport channels through the phospholipid bilayer of the nerve cell membrane). This analogous comparison continues the case for peaks within the conventional EEG frequency spectrum having a quantum resonance origin (note that interactions such as optical collisions, LACT, and laser induced collisional energy transfer (LICET), which are further exemplified by examples below, are considered closely related processes $[83,87]$, and so are all generically referred to as optical collisions for ongoing discussion).

Many optical collision systems involving chemical elements of the action potential's ion transport process have in fact long been studied ${ }^{6}$ with select examples including: $\mathrm{H}^{+}$-Na [86]; Na-Na at ultracold temperatures [82]; Na-noble gas [85]; He-He and He-other noble gases, including at room temperature [83,84,88]; Sr-Ca [89]; Na-Ca [90]; Rb$\mathrm{Na}$ [80]; and Rb-K [91].

As per Subsection 5.5, within the formalism of such optical collisions the total molecular potential often includes a centripetal term (that relates to the centripetal motion of interacting atoms), $V_{c}$, such that $V_{c} \propto J(J+1)$, where $J$ is a quantum number labelling the eigenvalues (energy solutions) associated with the total molecular angular momentum. For many of these systems, reaction cross-section profiles are oscillatory (as a function of inputted energy) with strong, specific and complex shape resonances that are identifiable with particular values of $J$. Such resonance characteristics are most pronounced in systems of relatively low mass, low temperature (i.e., approximately room temperature and below) and/or close internuclear separation, and accordingly are especially apparent within the above Na-noble gas, He-He, and Na-Na examples. In addition to the elemental and environmental contextual analogies that can subsequently be drawn, resonances within the above-described systems also share several physical characteristics with $F T^{\prime}$ spectra:

- Like $F T^{\prime}$ spectra, shape resonances within reaction crosssection profiles can be constructed from harmonics, that span and constitute a frequency spectrum, with oscillatory decay.

- Shape resonances typically involve rapid $90^{\circ}$ phase shifts and the present study identifies a primary Family based upon $\Delta \phi_{c}=90^{\circ}$ (and recall the dominant $\Delta \phi=90^{\circ}$ in figure $7 d)$.

\footnotetext{
${ }^{6}$ Many comparable studies may be cited (e.g., see review [78]) but are too numerous to list, with the vintage of presented examples (from the 1970s onwards) highlighting the depth of understanding and establishment of associated quantum resonances.
} 
- Shape resonances and the EEG (and therefore $F T^{\prime}$ spectra) share electric dipole origins. That is, it is often appropriate to describe the molecular binding potentials (e.g., van der Walls, adiabatic) of optical collisions as "dipole-dipole-type" (representative of weak interactions responsible for a departure from ideal gas behaviour). These potentials arise because the interacting (typically stable) atoms or molecules often possess permanent diploes due to electron affinity considerations (or even oscillating dipoles due to fluctuating quantum mechanical behaviour). For the EEG, dipole-type interactions play a role within the ion transport processes of the action potential, not just in terms of interactions between ions but also within the complex manner of ion transport channel gating across the nerve cell membrane. Not surprisingly then, dipole approximations to cortical electric current sources (or more specifically dipole moment per unit volume approximations of complex current distributions throughout cortical regions) provide the basis for most realistic EEG simulation models which assume (including for geometric convenience) that EEG sources consist of thousands of dipoles orientated perpendicular to the cortical surface $[4,5]$.

Additional analogies based upon mathematical functionalities may also be drawn. For example, parabolic and linear functionalities are identified with prominence within the present study, while for magnetically tuneable Feshbach resonances, in the vicinity of expected resonance where two channels are strongly coupled (and in the limit of large positive scattering length), the binding energy (or molecular state energy) depends parabolically on the detuning of the applied magnetic field, while away from the region of classical resonance the energy varies linearly with the magnetic detuning [78].

In summary, the following characteristics of showcased resonance-affected optical collisions draw analogy with the EEG on the basis of: (i) shared characteristics with the $J$ dependent oscillatory nature of reaction cross-sections (that may for example be derived from scattering matrices constructed of oscillatory spherical Bessel functions); (ii) the involved chemical elements (having overlap with elements of the action potential's ion transport processes) and potentials; and (iii) the addition of energy to provide molecular transition completion or excitation, including charge transfer (cellular energy must directly or indirectly be inputted for action potential charge transfer).

It can thus be stated that while the ion transport processes of the action potential may involve physical conditions dissimilar to the previously cited subset of example optical collisions (e.g., dissimilar temperature, matter phase, manner of energy excitation such as cellular rather than laser), based on analogous comparisons made the existence of oscillatory resonances within a fully quantum treatment of the action potential would be unsurprising, if not expected, and indeed such a treatment is considered necessary to fully interpret $F T^{\prime}$ resonances of the EEG into the future.

\section{REFERENCES}

1. Aron J. 2015 DNA in glass - the ultimate archive. New Scientist 225, 15.

2. Simeoni RJ. A discrete oscillator phase noise effect applied within phase-shift keying RF digital signal modulation. In: $9^{\text {th }}$ International Conference on Signal Processing and Communication Systems; 2015 December 14-16; Cairns, Australia. p. 1-9. (https://doi.org/10.1109/ICSPCS.2015.7391745)

3. Rennie CJ, Robinson PA, Wright JJ. 2002 Unified neurophysical model of EEG spectra and evoked potentials. Biol. Cybern. 86, 457-471. (https://doi.org/10.1007/s00422-002-0310-9)

4. Srinivasan R, Winter WR, Nunez PL. 2006 Source analysis of EEG oscillation using high-resolution EEG and MEG. Prog. Brain Res. 159, 29-42. (https://doi.org/10.1016/s0079-6123(06)59003-x)

5. Nunez PL, Nunez MD, Srinivasan R. 2019 Multi-scale neural sources of EEG: genuine, equivalent, and representative. A tutorial review. Brain Topogr. 32, 193-214. (https://doi.org/10.1007/s10548-019-00701-3)

6. Salimpoor VN, Benovoy M, Larcher K, Dagher A, Zatorre RJ. 2011 Anatomically distinct dopamine release during anticipation and experience of peak emotion to music. Nat. Neurosci. 14, 257-262. (https://doi.org/10.1038/nn.2726)

7. Chandra ML, Levitin DJ.2013 The neurochemistry of music. Trends Cogn. Sci. 17, 179-193. (https://doi.org/10.1016/j.tics.2013.02.007)

8. Stender J, Gosseries O, Bruno M, Charland-Verville V, Vanhaudenhuyse A, Demertzi A, Chantelle C, Thonnard M, Thibaut A, Heine L, et al. 2014 Diagnostic precision of PET imaging and functional MRI in disorders of consciousness: a clinical validation study. Lancet 384, 514-522. (https://doi.org/10.1016/S0140-6736(14)60042-8)

9. Kanduri C, Raijas P, Ahvenainen M, Philips AK, Ukkola-Vuoti L, Lähdesmäki H, Järvelä I. 2015 The effect of listening to music on human transcriptome. PeerJ 3:e830. (https://doi.org/10.7717/peerj.830)

10. Itthipuripat S, Sprague TC, Serences JT. 2019 Functional MRI and EEG index complementary attentional modulations. J. Neurosci. 39, 61626179. (https://doi.org/10.1523/JNEUROSCI.2519-18.2019)

11. Noor NSEM, Ibrahim H. 2020 Machine learning algorithms and quantitative electroencephalography predictors for outcome prediction in traumatic brain injury: a systematic review. ACCESS 8, 102075-102092. (https://doi.org/10.1109/ACCESS.2020.2998934)

12. Blasco MA, Redleaf MI. 2014 Cochlear implantation in unilateral sudden deafness improves tinnitus and speech comprehension: meta-analysis and systematic review. Otol. Neurotol. 35, 1426-1432. (https://doi.org/10.1097/MAO.0000000000000431)

13. Lou YH-L, da Cruz L. 2014 A review and update on the current status of retinal prosthesis. Br. Med. Bull. 109, 31-44. (https://doi.org/10.1093/bmb/ldu002)

14. Krucoff MO, Rahimpour S, Slutzky MW, Edgerton VR, Turner DA. 2016 Enhancing nervous system recovery through neurobiologics, neural interface training, and neurorehabilitation. Front. Neurosci. 10, 584. (https://doi.org/10.3389/fnins.2016.00584)

15. Gilbert F, Cook M, O'Brian T, Illes J. 2019 Embodiment and estrangement: results from a first-in-human "intelligent BCl" trial. Sci. Eng. Ethics 25, 83-96. (https://doi.org/10.1007/s11948-017-0001-5)

16. Drew L. 2019 The ethics of brain-computer interfaces. Nature 571, S19S21. (https://doi.org/10.1038/d41586-019-02214-2)

17. Baldermann JC, Schüler T, Huys D, Becker I, Timmermann L, Jessen F, Visser-Vandewalle V, Kuhn J. 2016 Deep brain stimulation for Tourettesyndrome: A systematic review and meta-analysis. Brain Stimul. 9, 296304. (https://doi.org/10.1016/j.brs.2015.11.005)

18. Keifer Jr OP, Riley JP, Boulis NM. 2014 Deep brain stimulation for chronic pain: intracranial targets, clinical outcomes, and trial design considerations. Neurosurg. Clin. N. Am. 25, 671-692. (https://doi.org/10.1016/j.nec.2014.07.009) 
19. Kisely S, Li A, Warren N, Siskind D. 2018 A systematic review and metaanalysis of deep brain stimulation for depression. Depress. Anxiety 35, 468-480. (https://doi.org/10.1002/da.22746)

20. Gaynes BN, Lloyd SW, Lux L, Gartlehner G, Hansen RA, Brode S, Jonas DE, Evans TS, Viswanathan M, Lohr KN, et al. 2014 repetitive transcranial magnetic stimulation for the treatment of depression: a systematic review and meta-analysis. J. Clin. Psychiat. 75, 477-489. (https://doi.org/10.4088/JCP.13r08815)

21. Finnigan SP, Walsh M, Rose SE, Chalk JB. 2007 Quantitative EEG indices of sub-acute ischaemic stroke correlate with clinical outcomes. Clin. Neurophysiol. 118, 2525-2532. (https://doi.org/10.1016/j.clinph.2007.021)

22. Cao T, Wan F, Wong CM, da Cruz JN, Hu Y. 2914 Objective evaluates of fatigue by EEG spectral analysis in steady-state visual evoked potential-based brain-computer interfaces. Biomed. Eng. Online 13, 28. (https://doi.org/10.1186/1475-925X-13-28)

23. Hagihira S. 2015 Changes in the electroencephalogram during anaesthesis and their physiological basis. Br. J. Anaesth. 115, i27-i31. (https://doi.org/10.1093/bja/aev212)

24. Nunez PL, Wingeier BM, Silberstein RB. 2001 Spatial-temporal structures of human alpha rhythms: theory, micro-current sources, multiscale measurements, and global binding of local networks. Hum. Brain Mapp. 13, 125-164. (https://doi.org/10.1002/hbm.1030)

25. Bowyer SM. 2016 Coherence a measure of the brain networks: past and present. Neuropsychiatr. Electrophysiol. 2, 1. (https://doi.org/10.1186/s40810-015-0015-7)

26. Schwarz G, Voit-Augustin H, Litscher G, Baumgartner A. 2003 Specific problems in interpretation of absolute values of spectral edge frequency (SEF) in comparison to bispectral index (BIS) for assessing depth of anesthesia. Internet J. Neuromonit. 3, 1-22. (https://doi.org/10.5580/12ca)

27. Billard V, Gambus PL, Chamoun N, Stanski DR, Shafer SL. 1997 A comparison of spectral edge, delta power, and bispectral index as EEG measures of alfentanil, propofol, and midazolam drug effect. Clin. Pharmacol. Ther. 61, 45-58. (https://doi.org/10.1016/S00099236(97)90181-8)

28. Stoeckel H, Schwilden H, Lauven P, Schüttler H. 1981 EEG indices for evaluation of depth of anaesthesia: the median of frequency distribution. Br. J. Anaesth. 53, 117P

29. Ning T, Bronzino JD. 1990 Autoregressive and bispectral analysis techniques: EEG applications. IEEE Eng. Med. Biol. March, 47-50. (https://doi.org/10.1109/51.62905)

30. Sigl JC, Chamoun NG. 1994 An introduction to bispectral analysis for the electroencephalogram. J. Clin. Monit. 10, 392-404. (https://doi.org/10.1007/BF01618421)

31. Lipton JM, Dabke KP, Alison JF, Cheng H, Yates L, Brown TIH. 1998 Use of the bispectrum to analyse properties of the human electrocardiograph. Austral. Phys. Eng. Sci. Med. 2, 1-10.

32. Muthuswamy J, Sherman DL, Thakor NV. 1999 Higher-order spectral analysis of burst patterns in EEG. IEEE Trans. Biomed. Eng. 46, 92-99. (https://doi.org/10.1109/10.736762)

33. Goldberger AL, Rigney DR, West BJ. 1990 Chaos and fractals in human physiology. Sci. Am. 262, 40-49. (https://doi.org/10.1038/scientificamerican0290-42)

34. Simeoni RJ, Mills PM. 2003 Does the Fibonacci sequence exist within our brain waves? The Physicist 40, 62-65.

35. Schieke K, Wacker M, Benninger F, Feucht M, Leistritz L, Witte H. 2015 Matching pursuit-based time-varient bispectral analysis and its application to biomedical signals. IEEE Trans. Biomed. Eng. 62, 19371948. (https://doi.org/10.1109/TBME:2015.2407573)

36. Nahm W, Stockmanns G, Petersen J, Gehring H, Konecny E, Kochs HD, Kochs E. 1999 Concept for an intelligent anaesthesia EEG monitor. Med. Inform. Internet. 24, 1-9. (https://doi.org/10.1080/146392399298492)

37. Schneider G, Gelb AW, Schmeller B, Tschakert R, Kochs E. 2003 Detection of awareness in surgical patients with EEG-based indices bispectral index and patient-based index. Br. J. Anaesth. 91, 329-335. (https://doi.org/10.1093/bja/aeg188)

38. Onuki K, Onuki N, Imamura T, Yamanishi Y, Yoshikawa S, Hagihira S Shimada J, Nagasaka H. 2001 Pentazocine increases bispectral index without surgical stimulation during nitrous oxide-sevoflurane. J. Anesth. 25, 946-949. (https://doi.org/10.1007/s00540-011-1224-2)
39. Hayashi K, Mukai N, Sawa T. 2014 Simultaneous bicoherence analysis of occipital and frontal electroencephalograms in awake and anesthetized subjects. Clin. Neurophysiol. 125, 194-201.

(https://doi.org/10.1016/j.clinph.2013.06.024)

40. Chua KC, Chandran V, Acharya UR, Lim CM. 2011 Application of highe order spectra to identify epileptic EEG. J. Med. Syst. 35, 1563-1571. (https://doi.org/10.1007/s10916-010-9433-z)

41. Schieke K, Wacker M, Piper D, Benninger F, Feucht M, Witte H. 2014 Time-variant, frequency-selective, linear and nonlinear analysis of heart rate variability in children with temporal and lobe epilepsy. IEEE Trans. Biomed. Eng. 61, 1798-1808. (https://doi.org/10.1109/TBME.2014.2307481)

42. Zhang JW, Zheng CX, Xie A. 2000 Bispectrum analysis of focal ischemic cerebral EEG signal using third-order recursion method. IEEE Trans. Biomed. Eng. 47, 352-359. (https://doi.org/10.1109/10.827296)

43. Li X, Li D, Voss LJ, Sleigh JW. 2009 The comodulation measure of neuronal oscillations with general harmonic wavelet bicoherence and application to sleep analysis. Neurolmage 48, 501-514. (https://doi.org/10.1016/j.neuroimage.2009.07.008)

44. Wacker M, Schiecke K, Putsche P, Eiselt M, Witte H. 2012 A processing scheme for time-variant phase analysis in EEG burst activity of premature and full-term newborns in quiet sleep: a methodological study. Biomed. Tech. (Berl.) 57, 491-505. (https://doi.org/10.1515/bmt-20120034)

45. Simeoni RJ, Mills PM. 2003 Quadriceps muscles vastus medialis obliques, rectus femoris and vastus lateralis compared via electromyogram bicoherence analysis. Austral. Phys. Eng. Sci. Med. 26 125-131. (https://doi.org/10.1007/BF03178782)

46. Al-Fahoum A, Al-Fraihat A, Al-Araida A. 2014 Detection of cardiac ischaemia using bispectral analysis approach. J. Med. Eng. Technol. 38, 311-316. (https://doi.org/10.3109/03091902.2014.925983)

47. Simeoni RJ, Mills PM. 2003 Bicoherence analysis of quadriceps electromyogram during isometric knee extension. Austral. Phys. Eng. Sci. Med. 26, 12-17. (https://doi.org/10.1007/BF03178691)

48. Simeoni RJ, Mills PM. Bispectral analysis of Alzheimer's electroencephalogram: a preliminary study. In: Allen B, Lovell N, editors, Proceedings of the World Congress on Medical Physics and Biomedical Engineering; 2003 August 24-29; Sydney, Australia

49. Lipton JM, Dabke KP. 1996 Bispectral properties of the simplest dissipative nonautonomous chaotic circuit. Int. J. Bifurcat. Chaos 6, 2419- 2425. (https://doi.org/10.1142/S0218127496001569)

50. Khandra L, Al-Fahoum AS, Binajjaj S. 2005 A quantitative analysis approach for cardiac arrhythmia classification using higher order spectral techniques. IEEE Trans. Biomed. Eng. 52, 1840-1845. (https://doi.org/10.1109/TBME.2005.856281)

51. Palva S, Palva JM. 2007 New vistas for $\alpha$-frequency band oscillations. Trends Neurosci. 30, 150-158. (https://doi.org/10.1016/j.tins.2007.02.001)

52. Raghuveer MR. 1990 Time domain approaches to quadratic phase coupling estimation. IEEE Trans. Automat. Contr. 35, 48-56. (https://doi.org/10.1109/9.45142)

53. Zhou G, Giannakis GB. 1995 Retrieval of self-coupled harmonics. IEEE Trans. Signal Process. 43, 1173-1186.

54. Schack B, White H, Helbig M, Schelenz Ch, Specht M. 2001 Timevariant non-linear phase-coupling analysis of EEG burst patters in sedated patients during electroencephalic burst suppression period. Clin Neurophysiol. 112, 1388-1399. (https://doi.org/10.1016/S13882457(01)00577-6)

55. Smedler E, Uhlén P. 2014 Frequency decoding of calcium oscillations. Biochem. Biophys. Acta. 1840, 964-969, 2014. (https://doi.org/10.1016/j.bbagen.2013.11.015)

56. Nayak K, 2002 Electroencephalogram (EEG) Data, SCRI, Florida State University. Originally available from http://www.scri.fsu.edu/ nayak/chaos/data.html.

57. Cameron JR, Skofronick JG. 1978 Medical physics, 1st ed. New York John Wiley and Sons, Inc. (https://doi.org/10.1002/jcu.1870060522)

58. Terzano MG, Parrino L, Sherieri A, Chervin R, Chokroverty S, Guilleminault C, Hirshkowitz M, Mahowald M, Moldofsky H, Rosa A, et al. 2001 Atlas, rules, and recording techniques for the scoring of cyclic alternating pattern (CAP) in human sleep. Sleep Med. 2, 537-553. (https://doi.org/10.1016/s1389-9457(01)00149-6) 
59. St. Vincent's University Hospital/University College Dublin. 2011 Sleep Apnea Database (revised version). (https://doi.org/10.13026/C26C7D)

60. Goldberger A, Amaral L, Glass L, Hausdorff J, Ivanov PC, Mark RG Mietus JE, Moody GB, Peng C-K, Stanley HE, et al. 2000 PhysioBank, PhysioToolkit, PhysioNet: components of a new research resource for complex physiological signals. Circulation [Online] 101, e215-e220. (https://doi.org/10.1161/01.CIR.101.23.e215)

61. Marcuse LV, Schneider M, Mortati KA, Donnelly KM, Arnedo V, Grant AC. 2008 Quantitative analysis of the EEG posterior-dominant rhythm in healthy adolescents. Clin. Neurophysiol. 119, 1778-1781. (https://doi.org/10.1016/j.clinph.2008.02.023)

62. Daniel WW. 1995 Biostatistics a foundation for analysis in the health sciences, 6th ed. New York: John Wiley and Sons, Inc.

63. Sklar B. 2001 Digital communications fundamentals and applications, 2nd ed. Upper Saddle River, New Jersey: Prentice Hall PTR.

64. Harris FJ. 1978 On the use of windows for harmonic analysis with the discrete Fourier transform. Proc. IEEE 66, 51-83.

65. Simeoni RJ. 2008 Noble gas magic numbers: from quarks to quasars, Austral. Math. Soc. Gaz. 35, 93-96.

66. Simeoni RJ. 2003 Bohr's model of atomic hydrogen extended to include electron rotational kinetic energy. Physics in Canada. 59, 309-311.

67. Bransden $\mathrm{BH}$, Joachain CJ. 1992 Physics of atoms and molecules. Essex: Longman Scientific and Technical.

68. Mack K. 2016 The pattern of matter. Cosmos 66, 35.

69. Gordan RL, Jacobs MS, Schuele CM, McAuley JD. 2015 Perspectives on the rhythm-grammar link and its implications for typical and atypical language development. Ann. N.Y. Acad. Sci. 1337, 16-25. (https://doi.org/10.1111/nyas.12683)

70. Gómez-Romero M, Jiménez-Palomares J, Rodriguez-Mansilla J, Flores Nieto A, Garrido-Ardila EM, González-López-Arza MV. 2017 Benefits of music therapy on behaviour disorders in subjects diagnosed with dementia: A systematic review. Neurología 32, 253-263. (https://doi.org/10.1016/j.nrl.2014.11.001)

71. Johnston B. 1964 Scalar order as a compositional resource. Perspect. New Music 2, 56-76. (https://doi.org/10.2307/832482)

72. Powell J. 2011 How music works: the science and psychology of beautiful sounds, from Beethoven to the Beatles and Beyond. New York: Little, Brown Spark.

73. Gero S, Whitehead H, Rendell L. 2016 Individual, unit and vocal clan level identity cues in sperm whale codas. R. Soc. Open. Sci. 3:150372,112. (https://doi.org/10.1098/ros.150372)

74. Rendell L, Whitehead H. 2003 Vocal clans in sperm whales (physeter macrocephalus). Proc. R. Soc. Lond. B 270, 225-231. (https://doi.org/10.1098/rspb.2002.2239)

75. Whitehead H, Antunes R, Gero S, Wong SNP, Engelhaupt D, Rendell L. 2012 Multilevel societies of female sperm whales (physeter macrocephalus) in the Atlantic and Pacific: why are they so different? Int. J. Primatol. 33, 1142-1164. (https://doi.org/10.1007/s10764-012-9598-z)

76. Mishima Y, Morisaka T, Itoh M, Matsuo I, Sakaguchi A, Miyamoto $Y$ 2015 Individuality embedded in the isolation calls of captive beluga whales (Delphinapterus leucas). Zoological Lett. 1, 1142-1164. (https://doi.org/10.1186/s40851-015-0028-x)

77. More RM, Gerjuoy E. 1973 Properties of resonance wave functions. Phys. Rev. A 7, 1288-1303. (https://doi.org/10.1103/PhysRevA.7.1288)

78. Chin C, Grimm R, Julienne P, Tiesinga E. 2010 Feshbach resonances in ultracold gases. Rev. Mod. Phys. 82, 1225-1289. (https://doi.org/10.1103/RevModPhys.82.1225)

79. Vahala LL, Julienne PS, Havey MD. 1986 Non-adiabatic theory of finestructure branching cross sections for $\mathrm{Na}-\mathrm{He}, \mathrm{Na}-\mathrm{Ne}$ and $\mathrm{Na}-\mathrm{Ar}$ optical collisions. Phys. Rev. A: At. Mol. Opt. Phys. 34, 1856-1868. (https://doi.org/10.1103/PhysRevA.34.1856)

80. Cheron B, Kucal H, Hennecart D. 1990 Laser-induced collisional energy transfer in a rubidium-sodium mixture: experiment and interpretation in the framework of the non-adiabatic collision theory. J.Phys. B: At. Mol. Opt. Phys. 23, 4281-4291. (https://doi.org/10.1088/0953-4075/23/23/011)

81. Bransden $\mathrm{BH}$, Joachain CJ. 1992 Physics of atoms and molecules. Essex: Longman Scientific and Technical.

82. Moerdijk AJ, Verhaar BJ, Axelsson A. 1995 Resonances in ultracold collisions of ${ }^{6} \mathrm{Li},{ }^{7} \mathrm{Li}$ and ${ }^{23} \mathrm{Na}$. Phys. Rev. A 51, 4852-4861. (https://doi.org/10.1103/PhysRevA.51.4852)
83. Simeoni RJ, Peach G, Whittingham IB. 1996 Theoretical study of a LICET-like process in singlet helium systems. J. Phys. B: At. Mol. Opt. Phys. 29, 5567-5582. (https://doi.org/10.1088/0953-4075/29/22/028)

84. Simeoni RJ, Peach G, Whittingham IB. 1997 A laser induced collisional energy transfer (LICET) process involving metastable helium. J.Phys. B: At. Mol. Opt. Phys. 30, 1071-1075. (https://doi.org/10.1088/09534075/30/41024)

85. Lavert-Ofir E, Shagam Y, Henson AB, Gersten S, Klos J, Zuchowski PS, Narevicius J, Narevicius E. 2014 Observation of the isotope effect in subkelvin reactions. Nat. Chem. 6, 332-335. (https://doi.org/10.1038./nchem.1857)

86. Hsu YP, Olsen RE. 1985 Satellite structure in laser-assisted chargetransfer cross sections. Phys. Rev. A 32, 2707-2711. (https://doi.org/10.1103/PhysRevA.32.2707)

87. Szudy J, Baylis WE. 1996 Profiles of line wings and rainbow satellites associated with optical and radiative collisions. Phys. Rep. 266, 199-225. (https://doi.org/10.1016/0370-1573(95)00054-2)

88. Simeoni RJ. 1996 A theoretical investigation of laser induced collisional energy transfer in rare gases. PhD thesis, Department of Physics, James Cook University of North Queensland, Australia.

89. Falcone RW, Green WR, White JC, Young JF, Harris SE. 1977 Observation of laser-induced inelastic collisions. Phys. Rev. A 15, 13331335. (https://doi.org/10.1103/PhysRevA.15.1333)

90. Debarre A. 1983 High-resolution study of light-induced collisional energy transfer in Na-Ca mixture. J. Phys. B: At. Mol. Phys. 16, 431-436. (https://doi.org/10.1088/0022-3700/16/3/017)

91. Cheron B, Lemery H. 1982 Observation of laser induced collisional energy transfer in a rubidium-potassium mixture. Opt. Commun. 42, 109112. (https://doi.org/10.1016/0030-4018(82)90376-5) 